\title{
EL ENDEMISMO EN LAS LILIOPSIDA MEXICANAS
}

\author{
Adolfo Espejo Serna \\ Universidad Autónoma Metropolitana-Iztapalapa, \\ División de Ciencias Biológicas y de la Salud, Departamento de Biología, \\ Apdo. postal 55-535, 09340, México, D.F., México. \\ aes@xanum.uam.mx
}

\section{RESUMEN}

Se presenta un listado actualizado de las Liliopsida endémicas de México. De las 4,542 especies silvestres que habitan en el territorio nacional, 2,010 son endémicas exclusivas y la cifra asciende a 2,764 si consideramos la ampliación de los límites a lo que Rzedowski denomina Megaméxico 3. Se incluyen datos relativos al número de taxa endémicos por familia, por género y por estado, así como otros análisis relativos a la representación del endemismo por forma biológica y algunos ejemplos de endemismo ecológico.

Palabras clave: endemismo, México, monocotiledóneas.

\begin{abstract}
An updated cheklist of the Liliopsida endemic to Mexico is presented. Of the 4,542 species of native monocots that inhabit the national territory, 2,010 are strict endemics. The number increases to 2,764 if we consider the Rzedowski's Megamexico 3 concept. Data about the number of endemic taxa by family, genera and state, and other analysis related to the endemic representation by life forms and some examples of ecological endemism are included.
\end{abstract}

Key words: endemism, Mexico, monocots.

Con entre 20,244 y 30,000 taxa de plantas vasculares (Toledo, 1988, 1994; Rzedowski, 1991a, 1993; Dirzo y Gómez, 1996; Magaña y Villaseñor, 2002; Villaseñor, 2003; Espejo-Serna et al., 2004a,b), México es el cuarto país con mayor fito- 
diversidad del mundo (Mittermeier y Goettsch, 1992). Además de la mera cantidad, otra medida significativa de la riqueza e importancia vegetal de una región es el número de especies endémicas a la misma (Rzedowski, 1962, 1991b), ya que dicha cifra constituye por sí sola un índice preciso de la peculiaridad de la flora de una región determinada. Las especies endémicas resultan de gran interés desde el punto de vista florístico e indican condiciones especiales de suelo, clima, regiones de aislamiento ecológico, etc. Son especialmente útiles para la determinación de áreas de protección ambiental (Villaseñor, 1991) y para la delimitación de provincias florísticas. Rzedowski (1991a, 1991b) estimó que el porcentaje de especies de fanerógamas endémicas al país debía estar cercano a 52\%, aunque señaló también la falta de un inventario depurado que permitiera contar con datos más precisos.

Por otra parte, cabe mencionar que existen pocos trabajos publicados relativos al endemismo de las monocotiledóneas para regiones o grupos taxonómicos específicos, entre los cuales podemos mencionar los de Rzedowski $(1962,1991 \mathrm{~b})$ a nivel nacional y los de García-Mendoza et al. (1994), Dávila et al. (2002), Méndez-Larios et al. (2004), Rodríguez-Jiménez et al. (2005), Villarreal-Quintanilla y Encina-Domínguez (2005) y Estrada-Castillón et al. (2010) a nivel regional. Actualmente contamos con diversos inventarios y catálogos que incluyen todas las especies de monocotiledóneas del país (Espejo y López-Ferrari, 2008;) o bien compilan los taxa de familias y o géneros completos pertenecientes a dicha clase de plantas (Hágsater y Salazar, 1990; Davidse et al., 1994; Vega Aviña et al., 2000; Hágsater y Soto, 2003, 2008; Espejo-Serna et al., 2004; Castillo-Campos et al., 2005; Dávila et al., 2006; Soto-Arenas et al., 2007; Diego y González, 2008; Téllez, 2008), condición que nos ha permitido hacer un estudio más detallado del elemento endémico de las Liliopsida mexicanas. El objetivo del presente documento es mostrar los resultados de dicho análisis.

Entre 1993 y 2000 publicamos el Listado de Referencia de las Monocotiledóneas Mexicanas (Espejo-Serna y López-Ferrari, 1993a, 1993b, 1994, 1995, 1996, 1997a, 1997b, 1998, 2000), el cual se planeó con el propósito de obtener un inventario completo, estandarizado y fidedigno de las especies de este grupo de angiospermas para México. Como resultado registramos para el país 4,029 especies (o 4,295 taxa incluyendo subespecies y variedades) nativas, agrupadas en 629 géneros y 53 familias (sensu Dahlgren et al., 1985). De entonces a la fecha, el conocimiento florístico y taxonómico de las monocotiledóneas ha avanzado de manera importante. Se han descrito nuevas especies (e. gr.. Croat, 1997; López-Ferrari et al., 1997; Starr, 1997; Castillo-Campos et al., 1998; Frame et al., 1999; Salazar, 1999; GarcíaMendoza, 2000; Finot et al., 2004; Rosen, 2009; Croat et al., 2010; Saarela et al., 2010; Sánchez-Ken, 2010; Kennedy y Ganders, 2011; Lascurain et al., 2011) o inclu- 
so géneros (e. gr.: Zomlefer y Judd, 2002; Rodríguez y Ortiz-Catedral, 2003; Salazar y Ballesteros-Barrera, 2010), y se han recircunscrito algunas familias y géneros (e. gr.: Smith 1995; Andersson y Chase, 2001; Stevens, 2001 onwards; Thiede, 2001; Caddick et al., 2002; Flagg et al., 2010; The Plant List, 2012; Tropicos.org., consultada en febrero 2012; World Checklist of Selected Plant Families (WCSP, 2012). Además, el número de recolecciones y registros ha aumentando considerablemente (e. gr.: Rodríguez et al., 2003). De igual manera, como consecuencia de diversas revisiones taxonómicas, algunas de las cuales incluyen análisis moleculares y estudios de filogenia, la delimitación genérica en algunas familias ha sufrido cambios notables. Tal es el caso de las familias Orchidaceae, Poaceae y Cyperaceae (e. gr.: Tucker, 1994; Judziewicz et al., 2000; Peterson et al., 2001; Espejo-Serna, 2002; Soreng et al., 2003; Strong, 2003; Zuloaga et al., 2003; Barfuss et al., 2005; GonzálezElizondo et al., 2008; Reznicek y González Elizondo, 2008; Muthama Muasya et al., 2009; Soreng et al., 2009; Thomas et al., 2009; Ruiz-Sanchez et al., 2011; Salazar et al., 2011). Durante los últimos 10 años hemos continuado el incremento y la actualización de la base de datos de las monocotiledóneas mexicanas, capturando información geográfica y taxonómica procedente de ejemplares de herbario, así como de protólogos, diversas revisiones, monografías y floras.

De acuerdo con los datos con los que contamos actualmente, sabemos que en México prosperan 4,562 especies silvestres de Liliopsida (4,629 taxa, contando subespecies y variedades), agrupadas en 576 géneros y 46 familias (Apéndice 2). Este valor representa un incremento de 533 especies (334 taxa) en relación a las que reportamos en el listado de referencia (Espejo-Serna y López-Ferrari, 1993-2000).

Del total de especies nativas, 2,010 (2,071 taxa), 44.05\% (44.73\%) son endémicas al país (Apéndice 1). Sin embargo, si añadimos a este conteo los taxa que, si bien presentes en México, extienden su área de distribución allende el norte y/o sur de las fronteras del país, a las regiones que Rzedowski (1991b) incluye en el concepto de Megaméxico 3, el número de especies endémicas asciende a 2,764 (2,842 taxa), es decir a $60.58 \%(61.39 \%)$ (Apéndice 1).

Las familias con más de 100 especies endémicas en México (Cuadro 1) son Orchidaceae (46.31\%), Bromeliaceae (72.5\%), Poaceae (26.41\%), Agavaceae (80.75\%) y Cyperaceae (24.93\%), y aquellas con más de $50 \%$ de sus especies endemitas en el país son Anthericaceae (84.61\%), Hyacinthaceae (83.33\%), Agavacae (80.75\%), Nolinaceae (77.35\%), Melanthiaceae (76.47\%), Amaryllidaceae (76.54\%), Bromeliaceae (72.5\%), Iridaceae (69.72\%), Dioscoreaceae (66.66\%), Alliaceae (63.79\%), Hypoxidaceae (60\%), Eriocaulaceae (57.14\%), Liliaceae (53.84\%) y Commelinaceae (54.47\%). Es notable que la gran mayoría de las familias con afinidad acuática o 
Cuadro 1. Familias de Liliopsida con especies endémicas de México.

\begin{tabular}{lcr|lcr}
\hline Familia & $\begin{array}{c}\text { Núm. } \\
\text { total de } \\
\text { especies } \\
\text { en México }\end{array}$ & $\begin{array}{c}\text { Núm. de } \\
\text { especies } \\
\text { endémicas de } \\
\text { México (\%) }\end{array}$ & Familia & $\begin{array}{c}\text { Núm. } \\
\text { total de } \\
\text { especies } \\
\text { en México }\end{array}$ & $\begin{array}{c}\text { Núm. de } \\
\text { especies } \\
\text { México (\%) }\end{array}$ \\
\hline Orchidaceae & 1263 & $585(46.31)$ & Liliaceae & 26 & $14(53.84)$ \\
Bromeliaceae & 400 & $290(72.5)$ & Eriocaulaceae & 21 & $12(57.14)$ \\
Poaceae & 1094 & $289(26.41)$ & Smilacaceae & 22 & $9(40.9)$ \\
Agavaceae & 239 & $193(\mathbf{8 0 . 7 5})$ & Hypoxidaceae & 10 & $6(60)$ \\
Cyperaceae & 413 & $103(24.93)$ & Marantaceae & 19 & $6(31.57)$ \\
Iridaceae & 109 & $76(69.72)$ & Hyacinthaceae & 6 & $5(\mathbf{8 3 . 3 3})$ \\
Commelinaceae & 123 & $67(54.47)$ & Alismaceae & 20 & $3(15)$ \\
Amaryllidaceae & 81 & $62(\mathbf{7 6 . 5 4})$ & Convallariacae & 12 & $3(25)$ \\
Anthericacae & 65 & $55(\mathbf{8 4 . 6 1})$ & Alstroemeriaceae & 5 & $2(40)$ \\
Dioscoreaceae & 75 & $50(66.66)$ & Heliconiaceae & 14 & $2(14.28)$ \\
Araceae & 127 & $45(35.43)$ & Juncaceae & 24 & $2(8.33)$ \\
Nolinaceae & 53 & $41(\mathbf{7 7 . 3 5})$ & Costaceae & 6 & $1(16.66)$ \\
Arecaceae & 95 & $40(42.1)$ & Cyclanthaceae & 6 & $1(16.66)$ \\
Alliaceae & 58 & $37(63.79)$ & Triuridaceae & 4 & $1(25)$ \\
Melanthiaceae & 34 & $26(\mathbf{7 6 . 4 7 )}$ & Xyridaceae & 4 & $1(25)$ \\
\hline
\end{tabular}

subacuática (e. gr.: Mayacaceae, Juncaginaceae, Hydrocharitaceae, Potamogetonaceae, Typhaceae y Zosteraceae) no tengan siquiera una especie endémica. También es importante señalar que ninguna familia puede considerarse como endémica, ni de México ni de Megaméxico.

De los 576 géneros de monocotiledóneas presentes en el país, 284 (49.3\%) cuentan con al menos un taxon endémico al mismo (Apéndice 2), mientras que 338 (58.68\%) tienen representantes en Megaméxico 3. Por otra parte, 34 de los 576 géneros (5.9\%) son totalmente endémicos de México (Cuadro 2) y aunque $21(61.76 \%)$ de dichos taxa cuentan con un solo representante, destacan Viridantha y Hemiphylacus por presentar 7 y 5 especies respectivamente. Los géneros de Liliopsida presentes en el país que cuentan con más de 20 endemitas se enlistan en el Cuadro 3, son de notar Tillandsia y Agave, que superan los 100 taxa endémicos para México y Hechtia, Echeandia, Muhlenbergia, Malaxis, Habenaria, Epidendrum, Carex y Dioscorea, que cuentan con 50 o más. Sin embargo, los géneros con más de $90 \%$ de especies en- 
Cuadro 2. Géneros de Liliopsida endémicos de México.

\begin{tabular}{lc|lc}
\hline Géneros & Núm. de especies & Géneros & Núm. de especies \\
\hline Viridantha & 7 & Gibasoides & 1 \\
Hemiphylacus & 5 & Matudanthus & 1 \\
Dandya & 4 & Karinia & 1 \\
Galeoglossum & 4 & Ainea & 1 \\
Svenkoeltzia & 4 & Fosteria & 1 \\
Thyrsanthemum & 3 & Alamania & 1 \\
Cypringlea & 3 & Artorima & 1 \\
Physogyne & 3 & Hintonella & 1 \\
Bessera & 2 & Mexipedium & 1 \\
Ursulaea & 2 & Microepidendrum & 1 \\
Colima & 2 & Papperitzia & 1 \\
Calibanus & 2 & Buchlomimus & 1 \\
Hagsatera & 2 & Cyclostachya & 1 \\
Prochnyanthes & 1 & Griffithsochloa & 1 \\
Diphalangium & 1 & Reederochloa & 1 \\
Jaimehintonia & 1 & Schaffnerella & 1 \\
Petronymphe & 1 & Sohnsia & 1 \\
\hline
\end{tabular}

démicas son Hymenocallis, Hechtia, Polianthes, Deiregyne, Schoenocaulon y Milla (Cuadro 4). Solo Hechtia es endémico de Megaméxico 3.

Si analizamos la distribución del endemismo considerando las formas biológicas presentes en las monocotiledóneas mexicanas (Cuadro 5), es posible observar que la mayor proporción de especies endemitas se encuentra en las geófitas $(26.41 \%)$ seguidas por las epífitas (25.87\%), las hierbas $(22.13 \%)$ y las rosulifolias $(14.72 \%)$. En el $10.87 \%$ restante se agrupan las demás formas de vida: arborescentes, trepadoras, acuáticas y saprófitas. Si ampliamos el análisis abarcando a Megaméxico 3 podemos observar que, en términos generales, las proporciones se mantienen (Cuadro 5), aunque es posible notar un incremento en el numero de epífitas y hierbas y un decremento en el de las las geófitas y las hierbas arrosetadas, lo cual es el resultado de la presencia de ambientes más cálidos y húmedos en la región centroamericana.

Del total de especies epífitas presentes en México, ca. 73.5\% (Espejo et al., en prep.) pertenecen a las Liliopsida, ocupando las orquídeas el primer lugar con 844 taxa, seguidas de lejos por las bromeliáceas con 225 y por las aráceas con 65 . 
Cuadro 3. Géneros de Liliopsida con más de 20 especies endémicas de México.

\begin{tabular}{lcc|lcr}
\hline Género & $\begin{array}{c}\text { Núm. } \\
\text { total de } \\
\text { especies } \\
\text { en México }\end{array}$ & $\begin{array}{c}\text { Núm. de } \\
\text { especies } \\
\text { endémicas de } \\
\text { México (\%) }\end{array}$ & Género & $\begin{array}{c}\text { Núm. } \\
\text { total de } \\
\text { especies } \\
\text { en México }\end{array}$ & $\begin{array}{r}\text { Núm. de } \\
\text { especies } \\
\text { endémicas de } \\
\text { Méxo (\%) }\end{array}$ \\
\hline Tillandsia & 232 & $172(74.13)$ & Tigridia & 36 & $31(86.11)$ \\
Agave & 128 & $113(88.28)$ & Anthurium & 45 & $29(64.44)$ \\
Hechtia & 58 & $55(94.82)$ & Sisyrinchium & 44 & $28(63.63)$ \\
Echeandia & 65 & $55(84.61)$ & Manfreda & 31 & $25(80.64)$ \\
Muhlenbergia & 117 & $54(46.15)$ & Encyclia & 42 & $25(59.52)$ \\
Malaxis & 68 & $52(76.47)$ & Tradescantia & 43 & $25(58.13)$ \\
Habenaria & 75 & $51(68)$ & Schoenocaulon & 25 & $23(92)$ \\
Epidendrum & 123 & $51(41.46)$ & Prosthechea & 46 & $24(52.17)$ \\
Carex & 124 & $51(41.12)$ & Oncidium & 37 & $23(62.16)$ \\
Dioscorea & 75 & $50(66.66)$ & Stelis & 59 & $23(38.98)$ \\
Lepanthes & 63 & $45(71.42)$ & Zephyranthes & 34 & $22(64.7)$ \\
Pitcairnia & 47 & $38(80.85)$ & Chamaedorea & 46 & $20(43.47)$ \\
Hymenocallis & 32 & $31(96.87)$ & & & \\
\hline
\end{tabular}

En consecuencia no resulta sorprendente observar que entre las monocotiledóneas con mayor número de representantes endémicos sobresalen Tillandsia, Epidendrum, Lepanthes, Anthurium, Encyclia, Prosthechea, Oncidium y Stelis (Cuadro 3), géneros que agrupan elementos mayormente epífitos.

Cientocuatro especies (5.17\%) de las monocotiledóneas endémicas de México son arborescentes (Cuadro 5). La gran mayoría pertenece a las familias Arecaceae, Nolinaceae y Agavaceae, y si bien su representación numérica es baja, sus poblaciones son muy abundates y constituyen elementos fundamentales en palmares, izotales y diversos tipos de matorrales xerófilos (Rzedowski, 1978). Es el caso de especies como Beaucarnea gracilis Lem., Brahea dulcis (Kunth) Mart., Cryosophila nana (Kunth) Salomon, Nolina parviflora (Kunth) Hemsl., Sabal mexicana Mart., S. pumos (Kunth) Burret, Yucca filifera Chabuad, Y. decipiens Trel., Y. periculosa Baker y Y. valida Brandegee, entre otras.

Algo semejante sucede en el caso de las hierbas perennes arrosetadas, las cuales forman parte importante de diversos matorrales rosetófilos, como por ejemplo Hechtia glomerata Zucc., H. confusa L.B. Sm., H. chichinautzensis Mart.-Co- 
Cuadro 4. Géneros de Liliopsida con mayor proporción de especies endémicas de México.

\begin{tabular}{lcr|lrr}
\hline Género & $\begin{array}{c}\text { Núm. } \\
\text { total de } \\
\text { especies } \\
\text { en México }\end{array}$ & $\begin{array}{c}\text { Núm. de } \\
\text { especies } \\
\text { endémicas de } \\
\text { México (\%) }\end{array}$ & Género & $\begin{array}{c}\text { Núm. } \\
\text { total de } \\
\text { especies } \\
\text { en México }\end{array}$ & $\begin{array}{c}\text { Núm. de } \\
\text { especies } \\
\text { enémicas de }\end{array}$ \\
\hline Hymenocallis & 32 & $31(\mathbf{9 6 . 9})$ & Dasylirion & 21 & $17(81)$ \\
Hechtia & 58 & $55(\mathbf{9 4 . 8})$ & Pitcairnia & 47 & $38(80.9)$ \\
Polianthes & 17 & $16(\mathbf{9 4 . 1})$ & Manfreda & 31 & $25(80.6)$ \\
Deiregyne & 14 & $13(\mathbf{9 2 . 9})$ & Barkeria & 15 & $12(80)$ \\
Schoenocaulon & 25 & $23(\mathbf{9 2})$ & Clowesia & 5 & $4(80)$ \\
Milla & 10 & $9(\mathbf{9 0})$ & Triniochloa & 5 & $4(80)$ \\
Agave & 128 & $113(88.3)$ & Tristachya & 5 & $4(80)$ \\
Habranthus & 8 & $7(87.5)$ & Gibasis & 14 & $11(78.6)$ \\
Otatea & 8 & $7(87.5)$ & Malaxis & 68 & $52(76.5)$ \\
Tigridia & 36 & $31(86.1)$ & Mormodes & 17 & $13(76.5)$ \\
Brahea & 14 & $12(85.7)$ & Schiedeella & 12 & $9(75)$ \\
Hesperaloe & 7 & $6(85.7)$ & Beschorneria & 8 & $6(75)$ \\
Echeandia & 65 & $55(84.6)$ & Beaucarnea & 8 & $6(75)$ \\
Laelia & 11 & $9(81.8)$ & Zeugites & 8 & $6(75)$ \\
\hline
\end{tabular}

rrea, Espejo \& López-Ferr., H. stenopetala Klotzsch, H. tehuacana B. L. Rob., H. zamudioi Espejo, López-Ferr. \& I. Ramírez, Agave horrida Jacobi, A. lechugilla Torr., etc. Otras especies son los constituyentes casi exclusivos de la vegetación rupícola que habita riscos, acantilados, cañones y peñascos como es el caso de Hechtia tillandsioides (André) L.B. Sm., H. nuusaviorum Espejo \& López-Ferr., Tillandsia grandis Schltdl., T. atroviolacea Ehlers \& Koide, T. rhodocephala Ehlers \& Koide, T. escahuascensis Espejo, López-Ferr., Ceja \& A. Mend., Viridantha mauryana (L.B. Sm.) Espejo, Agave dasylirioides Jacobi \& C. D. Bouché, A. wendtii Cházaro, A. pendula Schnittsp. y A. chazaroi Váquez \& O. M. Valencia.

Es interesante notar que en las Liliopsida endémicas de México la representación de especies acuáticas, subacuáticas (1.74\%) y saprófitas $(0.49 \%)$, al contrario de lo señalado por Rzedowski para la totalidad de las fanerógamas (1991b), es muy escasa, ya que en total alcanza apenas $2.23 \%$ (Cuadro 5).

En relación con los tipos de endemismo determinados por condiciones ecológicas, si bien no tenemos datos completos, cabe mencionar la preferencia de al- 
Cuadro 5. Distribución del endemismo de Liliopsida en México de acuerdo con sus formas biológicas.

\begin{tabular}{lcc}
\hline Forma de vida & Endémicas de México & Endémicas de Megaméxico 3 \\
\hline Arborescentes (A) & $104(5.17 \%)$ & $159(5.75 \%)$ \\
Epífitas (E) & $520(25.87 \%)$ & $773(27.96 \%)$ \\
Geófitas (G) & $531(26.41 \%)$ & $651(23.55 \%)$ \\
Hierbas (H) & $445(22.13 \%)$ & $679(24.56 \%)$ \\
Hierbas rosulifolias (HA) & $296(14.72 \%)$ & $347(12.55 \%)$ \\
Acuáticas y subacuáticas (ASA) & $35(1.74 \%)$ & $46(1.66 \%)$ \\
Saprófitas (SAP) & $10(0.49 \%)$ & $16(0.57 \%)$ \\
Trepadoras (T) & $69(3.43 \%)$ & $93(3.36 \%)$ \\
TOTAL & $2010(100 \%)$ & $2764(100 \%)$ \\
\hline
\end{tabular}

gunas especies por suelos yesosos. A manera de ejemplo es posible citar a Agave gypsophila Gentry, Aristida gypsophila Beetle, Calochortus mendozae Espejo, López-Ferr. et Ceja, Chondrosum chasei (Swallen) Clayton, Gibasis gypsophila B. L. Turner, Jaimehintonia gypsophila B. L. Turner, Muhlenbergia gypsophila Reeder et C. Reeder, Sisyrinchium zamudioi Espejo, López-Ferr. et Ceja, Sporobolus nealleyi Vasey y Tradescantia gypsophila B. L. Turner. Asimismo podemos decir que varios taxa de Poaceae prefieren suelos salinos. Entre ellos se encuentran Muhlenbergia villiflora Hitchc., Reederochloa eludens Soderstr. et H.F. Decker y Sporobolus spiciformis Swallen por mencionar solo unos cuantos.

Las familias Agavaceae y Nolinaceae, así como los géneros Hechtia y Hemiphylacus presentan elevados niveles de endemismo en las zonas áridas del país, constituyendo, como ya se mencionó, parte fundamental de comunidades vegetales como izotales y/o matorrales rosetófilos diversos (Rzedowski, 1978) en las cuales son los representantes fisonómicamente predominantes. Asimismo, las familias Orchidaceae, Bromeliaceae y Araceae aportan a los bosques mesófilos y en menor medida a los bosques tropicales un buen contingente de especies epífitas endémicas.

En lo que toca a la distribución geográfica, podemos decir que casi todos los estados del país cuentan con especies endémicas, aunque Oaxaca y Chiapas (Cuadro 6) son los que tienen el mayor número, ya sean exclusivas o compartidas con otras entidades federativas adyacentes. Solo Tlaxcala y el Distrito Federal no presentan endemitas exclusivos en su territorio. 
Cuadro 6. Especies de Liliopsida endémicas por estado.

\begin{tabular}{|c|c|c|c|}
\hline Estado & $\begin{array}{l}\text { Núm. de endémicas } \\
\text { exclusivas por estado }\end{array}$ & $\begin{array}{l}\text { Núm. de } \\
\text { endémicas por } \\
\text { estado }\end{array}$ & $\begin{array}{c}\text { Exclusividad } \\
(\%)\end{array}$ \\
\hline Oaxaca & 193 & 952 & 20.27 \\
\hline Jalisco & 118 & 680 & 17.35 \\
\hline Chiapas & 102 & 766 & 13.31 \\
\hline Guerrero & 81 & 535 & 15.14 \\
\hline Veracruz & 55 & 499 & 11.02 \\
\hline Estado de México & 39 & 429 & 9.09 \\
\hline Durango & 37 & 322 & 11.49 \\
\hline Michoacán & 34 & 519 & 6.55 \\
\hline Nuevo León & 30 & 205 & 14.63 \\
\hline San Luis Potosí & 28 & 303 & 9.24 \\
\hline Baja California Sur & 26 & 66 & 39.39 \\
\hline Baja California & 23 & 42 & 40.47 \\
\hline Coahuila & 20 & 206 & 9.7 \\
\hline Chihuahua & 19 & 291 & 6.52 \\
\hline Morelos & 18 & 315 & 5.71 \\
\hline Nayarit & 18 & 298 & 6.04 \\
\hline Hidalgo & 17 & 292 & 5.8 \\
\hline Sonora & 17 & 184 & 9.23 \\
\hline Colima & 16 & 153 & 10.45 \\
\hline Puebla & 15 & 410 & 3.65 \\
\hline Tamaulipas & 12 & 204 & 5.88 \\
\hline Sinaloa & 9 & 178 & 5.05 \\
\hline Querétaro & 8 & 148 & 5.4 \\
\hline Guanajuato & 7 & 178 & 3.93 \\
\hline Quintana Roo & 6 & 60 & 10 \\
\hline Zacatecas & 4 & 142 & 2.81 \\
\hline Aguascalientes & 4 & 115 & 3.47 \\
\hline Yucatán & 3 & 52 & 5.76 \\
\hline Tabasco & 2 & 79 & 2.53 \\
\hline Campeche & 1 & 57 & 1.75 \\
\hline Distrito Federal & 0 & 214 & 0 \\
\hline Tlaxcala & 0 & 50 & 0 \\
\hline
\end{tabular}


Por último, es conveniente señalar que de las 2,010 especies de Liliopsida endémicas al territorio nacional, 261 (12.98\%) se encuentran enlistadas en alguna categoría de protección de la Norma Oficial Mexicana NOM-059-SEMARNAT (Norma Oficial Mexicana, 2010) (Cuadro 7). Furcraea macdougallii Matuda, Echinodorus virgatus (Hook. et Arn.) Micheli y Laelia gouldiana Rchb.f., se consideran extintas en el medio silvestre. La gran mayoría de especies categorizadas bajo alguna forma de protección se concentran en tres familias: Orchidaceae, Arecaceae y Agavaceae, aunque las Bromeliaceae, Nolinaceae, Poaceae e Iridaceae también cuentan con un número importante de elementos amenazados (Cuadro 7).

Mora et al. (2011) estimaron que se han catalogado un total de 215,644 especies de plantas terrestres a nivel mundial y predijeron, mediante métodos estadísticos, que el total de las mismas en el planeta estará cercano a las 298,000 (+- 8,200). Es decir que, de acuerdo con dichos cálculos, quedarían por describir aproximadamente entre 74,156 y 90,556 especies de plantas terrestres (briofitas a angiospermas).

Cuadro 7. Número de especies de Liliopsida endémicas de México incluidas en la NOM-059SEMARNAT-2010.

\begin{tabular}{lccccc}
\hline Familia & Amenazadas & $\begin{array}{c}\text { Probablemente } \\
\text { extinta en el } \\
\text { medio silvestre }\end{array}$ & $\begin{array}{c}\text { En peligro } \\
\text { de extinción }\end{array}$ & $\begin{array}{c}\text { Sujetas a } \\
\text { protección } \\
\text { especial }\end{array}$ & Total \\
\hline Orchidaceae & 42 & 1 & 14 & 60 & 117 \\
Arecaceae & 32 & 0 & 3 & 9 & 44 \\
Agavaceae & 13 & 1 & 3 & 22 & 39 \\
Bromeliaceae & 13 & 0 & 0 & 0 & 13 \\
Nolinaceae & 11 & 0 & 0 & 1 & 12 \\
Poaceae & 2 & 0 & 5 & 3 & 10 \\
Iridaceae & 2 & 0 & 0 & 7 & 9 \\
Amaryllidaceae & 3 & 0 & 2 & 0 & 5 \\
Melanthiaceae & 0 & 0 & 0 & 4 & 4 \\
Alismaceae & 1 & 1 & 0 & 0 & 2 \\
Liliaceae & 0 & 0 & 0 & 2 & 2 \\
Alliacaee & 0 & 0 & 1 & 0 & 1 \\
Araceae & 1 & 0 & 0 & 0 & 1 \\
Pontederiaceae & 0 & 0 & 1 & 0 & 1 \\
Triuridaceae & 0 & 0 & 0 & 1 & 1 \\
\hline
\end{tabular}


Si consideramos que México alberga entre 10 y $11 \%$ de la riqueza vegetal del mundo (Espejo-Serna et al., 2004a,b, Magaña y Villaseñor, 2002), entonces restarían por describir para el país entre 7,415 y 9,055 taxa específicos, de los cuales entre 423 y 516 corresponderían a briofitas, entre 312 y 380 a pteridofitas, entre 47 y 57 a gimnospermas, entre 1,373 y 1,679 a monocotiledóneas y entre 5,260 y 6,423 a dicotiledóneas. En promedio nos faltarían por describir cerca de 8,235 especies de plantas terrestres, lo cual representa $30.79 \%$ de las 26,743 ya conocidas para México.

Las cifras anteriores parecen exageradas o cuando menos elevadas, sin embargo, es posible corroborarlas, revisando la página web de The Plant List (The Plant List, 2012). Allí se puede comprobar que en vez de las 215,644 especies encontradas por Mora et al. (2011), existen 289,900 especies reconocidas a nivel mundial y un número considerable $(247,880)$ de nombres sin evaluar, de los cuales con seguridad una buena parte corresponderá a especies válidas. Si hacemos un ejercicio sencillo redondeando el número de especies aceptadas a 325,000 y consideramos que $10 \%$ de las mismas corresponde a México, entonces esperaríamos para el país un total cercano o mayor a los 32,500 taxa, número que supera las 30,000 que alguna vez estimó Toledo (1988).

En el Cuadro 8 se recopila la información relativa al número de especies descritas con material mexicano desde el 1 de enero de 2000 hasta la fecha (e. gr.: Espejo Serna et al., 2001; Espejo Serna et al., 2002; López-Ferrari y Espejo Serna, 2002; Carrillo-Reyes et al., 2003; Rodríguez y Ortiz-Catedral, 2003; Espejo Serna et al., 2005; Espejo-Serna et al., 2007; Hunt, 2007; Téllez Valdés y Geeta, 2007; Alvarado-Cárdenas y García-Mendoza, 2008; Castillejos-Cruz y Solano, 2008; Espejo et al., 2008; Hernández-Sandoval et al., 2008; López-Ferrari et al., 2009; Rodríguez, 2009; Rosen, 2009; Croat et al., 2010; García-Mendoza, 2010; Saarela et al., 2010; Sánchez-Ken, 2010; López-Ferrari et al., 2011; Solano y Ríos-Gómez, 2011; Webb

Cuadro 8. Especies de plantas vasculares que se han descrito con material procedente de México desde el 1 de enero del 2000 hasta la fecha.

\begin{tabular}{lll}
\hline Grupo & Especies & Familias / Géneros \\
\hline Pteridofitas & $43(10 \mathrm{ssp})$. & en 12 familias y 22 géneros \\
Gimnospermas & $8(2 \mathrm{ssp})$. & en 3 familias y 4 géneros \\
Monocotiledóneas & $244(19 \mathrm{ssp})$. & en 15 familias y 83 géneros \\
Dicotiledóneas & $616(103 \mathrm{ssp})$. & en 75 familias y 248 géneros \\
TOTAL & $911(134 \mathrm{ssp})$.1045 taxa & en 108 familias y 355 géneros \\
\hline
\end{tabular}


y Salazar-Ceseña, 2011). En dicho período de tiempo se han dado a conocer 911 especies (más 134 subespecies y variedades) nuevas de plantas vasculares, repartidas, como se muestra en el Cuadro 8, en un promedio de 76 taxa por año. En lo que toca a las Liliopsida, han sido descritas 244 especies (más 19 subespecies y/o variedades) (Cuadro 9), es decir, cerca de 20 por año. Seguramente las exploraciones botánicas a regiones poco conocidas del país darán como resultado el hallazgo y la descripción de numerosas nuevas especies que serán, al menos en su mayoría, especies endémicas. Si seguimos al mismo ritmo y considerando que aún restan por describir cerca de 8,235 especies de plantas terrestres, entonces tomará 108 años terminar la descripción de los taxa faltantes de la flora nativa de México.

Sin embargo, existen diversos factores que tenemos que considerar en estos cálculos, como son entre otros, el bajo número de taxónomos con el que contamos, el costo que representa la descripción de cada especie nueva y los fondos disponibles para hacerlo, la dificultad de acceso a las regiones que quedan por explorar, los factores socioeconómicos y políticos de las regiones por estudiar, las políticas nacionales educativas y de investigación que con frecuencia subestiman la importancia del trabajo florístico y el apoyo a las colecciones científicas, etc. Debemos tomar en cuenta también, que las tasas de exterminio, de especies y de biomas completos, superan con mucho a las de descripción de las mismas. Es también importante señalar en este momento, que la gran mayoría de los taxa que se describirán en los próximos años, presentan con seguridad algún grado de rareza: o sus poblaciones son escasas y/o muy localizadas o bien son especies microendémicas, lo cual hace la tarea más importante pero más difícil.

Cuadro 9. Número de especies y de taxa subespecíficos de Liliopsida descritos con material procedente de México desde el 1 enero de 2000 hasta la fecha.

\begin{tabular}{lcc|lcc}
\hline Familia & Especies & Taxa & Familia & Especies & Taxa \\
\hline Agavaceae & 18 & 5 & Iridaceae & 10 & \\
Amaryllidaceae & 7 & & Liliaceae & 1 & \\
Araceae & 1 & & Marantaceae & 3 & \\
Bromeliaceae & 53 & 4 & Melanthiaceae & 1 & \\
Commelinaceae & 4 & & Nolinaceae & 5 & \\
Cyperaceae & 8 & & Orchidaceae & 110 & 9 \\
Dioscoreaceae & 1 & & Poaceae & 21 & 1 \\
Hypoxidaceae & 1 & & & & \\
\cline { 3 - 5 } & & & TOTAL & 244 & 19 \\
\hline
\end{tabular}




\section{AGRADECIMIENTOS}

Quiero agradecer al doctor Jerzy Rzedowski por la amable invitación que me hizo para publicar el presente trabajo en el número 100 de la revista Acta Botanica Mexicana, así como por la revisión crítica del trabajo. Deseo también expresar mi más sincera gratitud a la maestra Ana Rosa López-Ferrari quien, como siempre, aportó sus valiosas sugerencias y revisó cuidadosamente la versión final del manuscrito. A los curadores y/o personal de los siguientes herbarios A, AMES, AMO, BM, BR, CAS, CH, CHAP, CHIP, CICY, CIIDIR, CODAGEM, ECON, EBUM, ENCB, FCME, GH, HAL, HEM, HUAA, HUMO, IBUG, IEB, IZTA, JBSD, K, LE, LL, MA, MEXU, MICH, MINN, MO, NY, OAX, P, QMEX, SEL, SERO, TEX, UAMIZ, US, XAL, VT, W y WU por las facilidades brindadas para consultar el material depositado en sus colecciones.

\section{LITERATURA CITADA}

Alvarado-Cárdenas, L. O. y A. García-Mendoza. 2008. Una especie nueva de Habranthus (Amaryllidaceae, Hippeastreae) para la flora del valle de Tehuacán-Cuicatlán. Novon 18: 283-286.

Andersson, L. y M. W. Chase. 2001. Phylogeny and classification of Marantaceae. Bot. J. Linnean Soc. 135: 275-287.

Barfuss M. H. J., R. Samuel, W. Till y T. F. Stuessy. 2005. Phylogenetic relationships in subfamily Tillandsioideae (Bromeliaceae) based on DNA sequence data from seven plastid regions. Amer. J. Bot. 92: 337-351.

Caddick, L. R., P. Wilkin, P. J. Rudall, T. A. J. Hedderson y M. W. Chase 2002. Yams reclassified: a recircumscription of Dioscoreaceae and Dioscoreales. Taxon 51: 103114.

Carrillo-Reyes, P., R. Vega Aviña y R. Ramírez-Delgadillo. 2003. Agave rzedowskiana, a new species in subgenus Littaea (Agavaceae) from western Mexico. Brittonia 55: 240-244.

Castillejos-Cruz, C. y E. Solano. 2008. Manfreda bulbulifera (Agavaceae), especie nueva de México. Acta Bot. Mex. 82: 67-73.

Castillo-Campos, G., A. P. Vovides y M. Vázquez Torres. 1998. Una nueva especie de Stromanthe (Marantaceae) de Veracruz, México. Polibotánica 8: 13-19.

Castillo-Campos, G., M. E. Medina Abreo, P. D. Dávila Aranda y J. A. Zavala Hurtado. 2005. Contribución al conocimiento del endemismo de la flora vascular en Veracruz, México. Acta Bot. Mex. 73: 19-57.

Croat, T. B. 1997. A revision of Philodendron subgenus Philodendron (Araceae) for Mexico and Central America. Ann. Missouri Bot. Gard. 84: 311-704. 
Croat, T. B., T. Krömer y A. Acebey. 2010. Monstera florescanoana (Araceae), a new species from central Veracruz, Mexico. Rev. Mex. Biodiv. 81: 225-228.

Dahlgren, R. M. T., H. T. Clifford y P. F. Yeo. 1985. The families of the Monocotyledons. Springer Verlag. Berlín, Alemania. 520 pp.

Davidse, G., M. Sousa S. y A. O. Chater (eds.). 1994. Flora Mesoamericana. V. 6. Alismataceae a Cyperaceae. Instituto de Biología, Universidad Nacional Autónoma de México, Missouri Botanical Garden y The Natural History Museum (Londres). St. Louis, Missouri, USA. 543 pp.

Dávila, P., M. D. C. Arizmendi, A. Valiente-Banuet, J. L. Villaseñor, A. Casas y R. Lira. 2002. Biological diversity in the Tehuacán-Cuicatlán Valley, Mexico. Biodiv. Conserv. 11: 421-442.

Dávila, P., M. T. Mejía-Saules, M. Gómez-Sánchez, J. Valdés-Reyna, J. J. Ortiz, C. Morín, J. Castrejón y A. Ocampo. 2006. Catálogo de las gramíneas de México. Universidad Nacional Autónoma de México - Comisión Nacional para el Conocimiento y Uso de la Biodiversidad. México, D.F., México. 671 pp.

Diego, N. y S. González. 2008. Ciperáceas (Monocotiledóneas). In: Ocegueda, S. y J. Llorente-Bousquets (coords.). Capital natural de México. Catálogo taxonómico de especies de México. Vol. I. Conocimiento actual de la biodiversidad. Comisión Nacional para el Conocimiento y Uso de la Biodiversidad. México, D.F., México. cd1.

Dirzo, R. y G. Gómez 1996. Ritmos temporales de la investigación taxonómica de plantas vasculares en México y una estimación del número de especies conocidas. Ann. Missouri Bot. Gard. 83: 396-403.

Espejo-Serna, A. y A. R. López-Ferrari. 1993a. Las monocotiledóneas mexicanas, una sinopsis florística 1. Lista de referencia, parte I. Agavaceae, Alismaceae, Alliaceae, Alstroemeriaceae y Amaryllidaceae. Consejo Nacional de la Flora de México y Universidad Autónoma Metropolitana. México, D.F., México. 76 pp.

Espejo-Serna, A. y A. R. López-Ferrari. 1993b. Las monocotiledóneas mexicanas, una sinopsis florística 1. Lista de referencia, parte II. Anthericaceae, Araceae, Arecaceae, Asparagaceae Asphodelaceae y Asteliaceae. Consejo Nacional de la Flora de México, Universidad Autónoma Metropolitana y Comisión Nacional para el Conocimiento y Uso de la Biodiversidad. México, D.F., México. 70 pp.

Espejo-Serna, A. y A. R. López-Ferrari. 1994. Las monocotiledóneas mexicanas, una sinopsis florística 1. Lista de referencia, parte III. Bromeliaceae, Burmanniaceae, Calochortaceae y Cannaceae. Consejo Nacional de la Flora de México, Universidad Autónoma Metropolitana y Comisión Nacional para el Conocimiento y Uso de la Biodiversidad. México, D.F., México. 73 pp.

Espejo-Serna, A. y A. R. López-Ferrari. 1995. Las monocotiledóneas mexicanas, una sinopsis florística 1. Lista de referencia, parte IV. Commelinaceae, Convallariaceae, Costaceae, Cyclanthaceae y Cymodoceaceae. Consejo Nacional de la Flora de México, Universidad Autónoma Metropolitana y Comisión Nacional para el Conocimiento y Uso de la Biodiversidad. México, D.F., México. 49 pp.

Espejo-Serna, A. y A. R. López-Ferrari. 1996. Las monocotiledóneas mexicanas, una sinopsis florística 1. Lista de referencia, parte VI. Dioscoreaceae a Nolinaceae. Consejo Nacional de la Flora de México, Universidad Autónoma Metropolitana y Comisión Nacional para el Conocimiento y Uso de la Biodiversidad. México, D.F., México. 116 pp. 
Espejo-Serna, A. y A. R. López-Ferrari. 1997a. Las monocotiledóneas mexicanas, una sinopsis florística 1. Lista de referencia, parte V. Cyperaceae. Consejo Nacional de la Flora de México, Universidad Autónoma Metropolitana y Comisión Nacional para el Conocimiento y Uso de la Biodiversidad. México, D.F., México. 98 pp.

Espejo-Serna, A. y A. R. López-Ferrari. 1997b. Las monocotiledóneas mexicanas, una sinopsis florística 1. Lista de referencia, parte VII. Orchidaceae I. Consejo Nacional de la Flora de México, Universidad Autónoma Metropolitana y Comisión Nacional para el Conocimiento y Uso de la Biodiversidad. México, D.F., México. 90 pp.

Espejo-Serna, A. y A. R. López-Ferrari. 1998. Las monocotiledóneas mexicanas, una sinopsis florística 1. Lista de referencia, parte VIII. Orchidaceae II. Consejo Nacional de la Flora de México, Universidad Autónoma Metropolitana y Comisión Nacional para el Conocimiento y Uso de la Biodiversidad. México, D.F., México. 115 pp.

Espejo-Serna, A. y A. R. López-Ferrari. 2000. Las monocotiledóneas mexicanas, una sinopsis florística 1. Lista de referencia, partes IX-XI. Pandanaceae a Zosteraceae. Consejo Nacional de la Flora de México, Universidad Autónoma Metropolitana y Comisión Nacional para el Conocimiento y Uso de la Biodiversidad. México, D.F., México. 337 pp.

Espejo Serna, A., A. R. López-Ferrari y J. Ceja Romero. 2001. Two new species of Mexican Iridaceae. Acta Bot. Mex. 55: 21-27.

Espejo-Serna, A. 2002. Viridantha, un género nuevo de Bromeliaceae (Tillandsioideae) endémico de México. Acta Bot. Mex. 60: 25-35.

Espejo Serna, A., A. R. López-Ferrari, R. Jiménez Machorro, L. Sánchez Saldaña y J. García Cruz. 2002. Dos especies nuevas de orquídeas del estado de Morelos, México. Acta Bot. Mex. 61: 27-33.

Espejo-Serna, A., A. R. López-Ferrari e I. Salgado Ugarte. 2004a. A current estimate of angiosperm diversity in Mexico. Taxon 53: 127-130.

Espejo-Serna, A., A. R. López-Ferrari, I. Ramírez-Morillo, B. K. Holst, H. E. Luther y W. Till. 2004b. Checklist of Mexican Bromeliaceae with notes on species distribution and levels of endemism. Selbyana 25: 33-86.

Espejo Serna, A., A. R. López-Ferrari y J. Ceja Romero. 2005. Calochortus mendozae (Calochortaceae), una nueva especie de San Luis Potosí, México. Novon 15: 279281.

Espejo-Serna, A., A. R. López-Ferrari, I. Ramírez-Morillo y N. Martínez-Correa. 2007. Dos nuevas especies de Hechtia (Bromeliaceae) de México. Acta Bot. Mex. 78: 97-109.

Espejo, A. y A. R. López-Ferrari, 2008. Monocotiledóneas. In: Ocegueda, S. y J. LlorenteBousquets (coords.). Capital natural de México, catálogo taxonómico de especies de México. Vol. I. Conocimiento actual de la biodiversidad. Comisión Nacional para el Conocimiento y Uso de la Biodiversidad. México, D.F., México. cd1.

Espejo, A., A. R. López-Ferrari y W. Till. 2008. Dos nuevas especies de Tillandsia (Bromeliaceae) de México. Acta Bot. Mex. 85: 45-62.

Estrada-Castillón, E., L. Scott-Morales, J. Á. Villareal-Quintanilla, E. Jurado-Ybarra, M. Cotera-Correa, C. Cantú-Ayala y J. García-Pérez. 2010. Clasificación de los pastizales halófilos del noreste de México asociados con perrito de las praderas (Cynomys mexicanus): diversidad y endemismo de especies. Rev. Mex. Biodiv. 81: 401-416. 
Finot, V. L., P. M. Peterson, R. J. Soreng y F. O. Zuloaga. 2004. A revision of Tristeum, Peyritschia, and Sphenopholis (Poaceae: Aveninae) in Mexico and Central America. Ann. Missouri Bot. Gard. 91: 1-30.

Flagg, R. O., G. L. Smith y A. W. Meerow. 2010. New combinations in Habranthus (Amaryllidaceae) in Mexico and Southwestern U.S.A. Novon 20: 33-34.

Frame, D., A. Espejo y A. R. López-Ferrari. 1999. A conspectus of Mexican Melanthiaceae including a description of new taxa of Schoenocaulon and Zigadenus. Acta Bot. Mex. 48: 27-50.

García Mendoza, A. 2000. Revisión taxonómica de las especies arborescentes de Furcraea (Agavaceae) en México y Guatemala. Bol. Soc. Bot. Méx. 66: 113-129.

García-Mendoza, A. J. 2010. Especie nueva de Hymenocallis (Amaryllidaceae) de Oaxaca y Puebla, México. Rev. Mex. Biodiv. 81: 625-628.

García Mendoza, A., P. Tenorio Lezama y J. Reyes Santiago. 1994. El endemismo en la flora fanerogámica de la Mixteca Alta, Oaxaca, Puebla, México. Acta Bot. Mex. 27: 53-73.

González Elizondo, M. S., M. González Elizondo, J. A. Tena Flores, I. L. López Enríquez, A. A. Reznicek y N. Diego-Pérez. 2008. Sinopsis de Scirpus s. 1. (Cyperaceae) para México. Acta Bot. Mex. 82: 15-41.

Hágaster, E. y G. A. Salazar (eds.). 1990. Icones Orchidacearum. Fasc. 1. Orchids of Mexico. Part 1. Herbario AMO, México, D.F. http://www.herbarioamo.org/index_archivos/ Page284.htm

Hágsater, E. y M. Soto (eds.). 2003. Icones Orchidacearum. Fasc. 5 y 6. Orchids of Mexico Parts 2 y 3. Herbario AMO, México, D.F. http://www.herbarioamo.org/index_ archivos/Page284.htm

Hágsater, E. y M. Soto (eds.). 2008. Icones Orchidacearum. Fasc. 10. Orchids of Mexico Part 4. Herbario AMO. México, D.F. http://www.herbarioamo.org/index_archivos/ Page284.htm

Hernández-Sandoval, L., R. Orellana y G. Carnevali. 2008. Two new species of Manfreda Salisb. (Agavaceae) from the Yucatán Península, Mexico. J. Torrey Bot. Soc. 135: 168-177.

Hunt, D. 2007. A new species of Tradescantia (Commelinaceae) from Mexico. Kew Bull. 62: $141-142$.

Judziewicz, E. J., R. J. Soreng, G. Davidse, P. M. Peterson, T. S. Filgueiras y F. O. Zuloaga. 2000. Catalogue of New World grasses (Poaceae): I. Subfamilies Anomochlooideae, Bambusoideae, Ehrhartoideae, and Pharoideae. Contr. U.S. Natl. Herb. 39: 1-128.

Kennedy, H. y F. R. Ganders. 2011. Calathea matudae and C. sousandradeana, new species of Marantaceae from Mexico and Northern Central America. Novon 21: 58-65.

Lascurain, M., H. Oliva Rivera y S. Avendaño. 2011. Calathea leonoriae (Marantaceae), una nueva especie de Veracruz, México. Novon 21: 66-68.

Lopez-Ferrari, A. R., A. Espejo Serna y J. Ceja Romero 1997. Dos nuevas especies de Commelina (Commelinaceae) mexicanas. Acta Bot. Mex. 41: 9-16.

López-Ferrari, A. R. y A. Espejo Serna. 2002. Tigridia potosina (Iridaceae: Tigridieae) una nueva especie de la Sierra de Álvarez, San Luis Potosí, México. Acta Bot. Mex. 61: 35-40.

López-Ferrari, A. R., A. Espejo-Serna y J. Ceja-Romero. 2009. Tres nuevas especies de Commelina (Commelinaceae) del centro de México. Acta Bot. Mex. 87: 71-81. 
López-Ferrari, A. R ., A. Espejo-Serna, J. Ceja-Romero y A. Mendoza-Ruiz. 2011. Aechmea aenigmatica (Bromeliaceae; Bromelioideae) una nueva especie del estado de Oaxaca, México. Acta Bot. Mex. 95: 1-9.

NORMA OFICIAL MEXICANA NOM-059-SEMARNAT-2010. Protección ambientalEspecies nativas de México de flora y fauna silvestres-Categorías de riesgo y especificaciones para su inclusión, exclusión o cambio-Lista de especies en riesgo. 2010. DIARIO OFICIAL. Jueves 30 de diciembre de 2010 (segunda sección): 1-77.

Magaña, P. y J. L. Villaseñor. 2002. La flora de México ¿Se podrá conocer completamente? Ciencias 66: 24-26.

Méndez-Larios, I., E. Ortiz y J. L. Villaseñor. 2004. Las Magnoliophyta endémicas de la porción xerofítica de la provincia florística del Valle de Tehuacán-Cuicatlán, México. Anales Inst. Biol. Univ. Nac. México. Ser. Bot. 75: 87-104.

Mittermeier, R. y C. Goettsch. 1992. La importancia de la diversidad biológica de México. In: Sarukhán, J. y R. Dirzo (comps.). México ante los retos de la biodiversidad. Comisión Nacional para el Conocimiento y Uso de la Biodiversidad. México, D.F., México. pp. 43-55.

Mora, C., D. P. Tittensor, S. Adl, A. G. B. Simpson y B. Worm. 2011. How many species are there on Earth and in the Ocean? PloS Biology 9: 1-8.

Muthama Muasya, A., D. A. Simpson, G. A. Verboom, P. Goetghebeur, R. F. C. Naczi, M. W. Chase y E. Smets. 2009. Phylogeny of Cyperaceae based on DNA sequence data: Current progress and future prospects. Bot. Rev. 75: 2-21.

Peterson, P. M., R. J. Soreng, G. Davidse, T. S. Filgueiras, F. O. Zuloaga y E. J. Judziewicz. 2001. Catalogue of New World grasses (Poaceae): II. Subfamily Chloridoideae. Contr. U.S. Natl. Herb. 41: 1-255.

Reznicek, A. A. y M. S. González Elizondo. 2008. Cypringlea (Cyperaceae) revisited, a new combination and status. Acta Bot. Mex. 83: 13-23.

Rodríguez, A. 2009. Manfreda parva (Agavaceae), especie nueva del estado de Guerrero, México. Acta Bot. Mex. 88: 1-8.

Rodríguez, A. y L. Ortiz-Catedral. 2003. Colima (Tigridieae: Iridaceae), a new genus from Western Mexico and a new species Colima tuitensis from Jalisco. Acta Bot. Mex. 65: 51-60.

Rodríguez, A., L. Ortiz-Catedral y E. Heaton. 2003. Tres nuevas localidades de tigridias endémicas de México: Tigridia bicolor, T. matudae y T. vanhouttei ssp. roldanii. Acta Bot. Mex. 62: 1-8.

Rodríguez-Jiménez, C., R. Fernández-Nava, M. L. Arreguín-Sánchez y A. RodríguezJiménez. 2005. Plantas vasculares endémicas de la cuenca del río Balsas, México. Polibotánica 20: 73-99.

Rosen, D. J. 2009. Eleocharis gonzaleziae (Cyperaceae), a new species from Northern Mexico. Novon 19: 511-514.

Ruiz-Sanchez, E., V. Sosa, M. T. Mejía-Saules, X. Londoño y L. G. Clark. 2011. A taxonomic revision of Otatea (Poaceae: Bambusoideae: Bambuseae) including four new species. Syst. Bot. 36: 314-336.

Rzedowski, J. 1962. Contribuciones a la fitogeografía florística e histórica de México. I. Algunas consideraciones acerca del elemento endémico en la flora mexicana. Bol. Soc. Bot. Méx. 27: 52-56. 
Rzedowski, J. 1978. La vegetación de México. Limusa. México, D.F., México. 432 pp.

Rzedowski, J. 1991a. Diversidad y orígenes de la flora fanerogámica de México. Acta Bot. Mex. 14: 3-21.

Rzedowski, J. 1991b. El endemismo en la flora fanerogámica mexicana: una apreciación analítica preliminar. Acta Bot. Mex. 15: 47-64.

Rzedowski, J. 1993. Diversity and origins of the phanerogamic flora of Mexico. In: Ramamoorthy, T. P., R. Bye, A. Lot y J. Fa (eds.). Biological diversity of Mexico: Origins and distribution. Oxford Univ. Press, New York, USA. pp. 129-144.

Saarela, J. M., P. M. Peterson, S. González Elizondo y D. J. Rosen. 2010. Eleocharis cryptica (Cyperaceae), a dwarf new species from Durango, Mexico. Brittonia 62: 233-238.

Salazar, G. A. 1999. Novelties in Mexican Orchidaceae, mainly from the UxpanapaChimalapa region, Veracruz and Oaxaca. Anales Inst. Biol. Univ. Nac. México, ser. Bot. 70: 1-12.

Salazar, G. A. y C. Ballesteros-Barrera. 2010. Sotoa, a new genus of Spiranthinae (Orchidaceae) from Mexico and the southern United States. Lankesteriana 9: 491504.

Salazar, G. A., L. I. Cabrera y C. Figueroa. 2011. Molecular phylogenetics, floral convergence and systematics of Dichromanthus and Stenorrhynchos (Orchidaceae: Spiranthinae). Bot. J. Linn. Soc. 167: 1-18.

Sánchez-Ken, J. G. 2010 Hypoxis colliculata (Hypoxidaceae), a new species from Mexico and a key to the American species with black seeds. Acta Bot. Mex. 92: 1-9.

Smith, S. G. 1995. New combinations in North American Schoenoplectus, Bolboschoenus, Isolepis, and Trichophorum (Cyperaceae). Novon 5: 97-102.

Solano, E. y R. Ríos-Gómez. 2011. Polianthes zapopanensis (Agavaceae), una especie nueva de Jalisco, México. Brittonia 63: 70-74.

Soreng, R. J., P. M. Peterson, G. Davidse, E. J. Judziewicz, F. O. Zuloaga, T. S. Filgueiras y O. Morrone. 2003. Catalogue of New World grasses (Poaceae): IV. Subfamily Pooideae. Contr. U.S. Natl. Herb. 48: 1-730.

Soreng, R. J., G. Davidse, P. M. Peterson, F. O. Zuloaga, E. J. Judziewicz, T. S. Filgueiras y O. Morrone. First published 13 January 2000. Data will be under continuous revision after this time. This page last revised 6 Jan. 2009. Catalogue of new world grasses (Poaceae). On line version: http://www.tropicos.org/Project/CNWG

Soto-Arenas, M. A., E. Hágsater, R. Jiménez Machorro, G. A. Salazar Chávez, R. Solano Gómez, R. Flores González e I. Ruiz Contreras. sin fecha (2007). Las orquídeas de México. Catálogo Digital. Interactive Multimedi CD, Win-Mac. Herbario AMO. Instituto Chinoín. México, D.F., México.

Starr, G. 1997. A revision of the genus Hesperaloe (Agavaceae). Madroño 44: 282-296.

Stevens, P. F. (2001 onwards). Angiosperm Phylogeny Website. Version 9, June 2008 [and more or less continuously updated since]. http://www.mobot.org/MOBOT/research/ APweb/. Page last updated: 03/22/2012 14:51:20

Strong, M. T. 2003. Cypringlea, a new genus of Cyperaceae from Mexico. Novon 13: 123132.

Téllez Valdés, O. y R. Geeta. 2007. Dioscorea howardiana, a new species in Dioscorea section Trigonobasis (Dioscoreaceae). Brittonia 59: 370-373. 
Téllez, O. 2008. Dioscoreáceas (Monocotiledóneas). In: Ocegueda, S. y J. LlorenteBousquets (coords.). Capital natural de México, catálogo taxonómico de especies de México. Vol. I: Conocimiento actual de la biodiversidad. Comisión Nacional para el Conocimiento y Uso de la Biodiversidad. México, D.F., México. cd1.

The Plant List. 2012. Version 1. Published on the Internet. http://www.theplantlist.org/ (accessed March-April).

Thiede, J. 2001. Agavaceae, In: Eggli, U. (ed.). Illustrated handbook of succulent plants. Springer-Verlag. Berlin-Heidelberg, Alemania. pp. 5-100.

Thomas, W. W., A. C. Araújo y M. V. Alves. 2009. A preliminary molecular phylogeny of the Rhynchosporeae (Cyperaceae). Bot. Rev. 75: 22-29.

Toledo, V. M. 1988. La diversidad biológica de México. Ciencia y Desarrollo 81: 17-30.

Toledo, V. M. 1994. La diversidad biológica de México, nuevos retos para la investigación en los noventas. Ciencias 34: 43-59.

Tropicos.org. Feb. 2012. Missouri Botanical Garden. http://www.tropicos.org

Tucker, G. C. 1994. Revision of the Mexican species of Cyperus (Cyperaceae). Syst. Bot. Monogr. 43: 1-213.

Vega Aviña, R., H. Aguiar Hernández, J. A. Gutiérrez García, J. A. Hernández Vizcarra, I. F. Vega López y J. L. Villaseñor. 2000. Endemismo regional presente en la flora del municipio de Culiacán, Sinaloa, México. Acta Bot. Mex. 53: 1-15.

Villarreal-Quintanilla, J. Á. y J. A. Encina Domínguez. 2005. Plantas vasculares endémicas de Coahuila y algunas áreas adyacentes, México. Acta Bot. Mex. 70: 1-46.

Villaseñor, J. L. 1991. Las Heliantheae endémicas a México: una guía hacia la conservación. Acta Bot. Mex. 15: 29-46.

Villaseñor, J. L. 2003. Diversidad y distribución de las Magnoliophyta de México. INCI 28: 160-167.

WCSP. 2012. World Checklist of Selected Plant Families. Facilitated by the Royal Botanic Gardens, Kew. Published on the Internet; http://apps.kew.org/wcsp/ Retrieved 201103-1 - 04-30.

Webb, R. H. y J. M. Salazar-Ceseña. 2011. Agave turneri (Agavaceae), a new species from northeastern Baja California, Mexico. Brittonia 63: 203-210.

Zomlefer, W. F. y W. S. Judd. 2002. Resurrection of segregates of the polyphyletic Genus Zigadenus s. 1. (Liliales: Melanthiaceae) and resulting new combinations. Novon 12: 299-308.

Zuloaga, F. O., O. Morrone, G. Davidse, T. S. Filgueiras, P. M. Peterson, R. J. Soreng y E. Judziewicz. 2003. Catalogue of New World grasses (Poaceae): III. Subfamilies Panicoideae, Aristidoideae, Arundinoideae, and Danthonioideae. Contr. U. S. Natl. Herb. 46: 1-662. 


\section{APÉNDICE 1}

Catálogo de las Liliopsida endémicas de México

Simbología: Megaméxico 1 $=*$ Megaméxico $2=* * \quad$ Megaméxico $3=* * *$ Aguascalientes (AGS), Baja California (BC), Baja California Sur (BCS), Campeche (CAM), Chiapas (CHIS), Chihuahua (CHIH), Coahuila (COAH), Colima (COL), Distrito Federal (DF), Durango (DGO), Estado de México (MÉX), Guanajuato (GTO), Guerrero (GRO), Hidalgo (HGO), Jalisco (JAL), Michoacán (MICH), Morelos (MOR), Nayarit (NAY), Nuevo León (NL), Oaxaca (OAX), Puebla (PUE), Querétaro (QRO), Quintana Roo (QROO), San Luis Potosí (SLP), Sinaloa (SIN), Sonora (SON), Tabasco (TAB), Tamaulipas (TAM), Tlaxcala (TLX), Veracruz (VER), Yucatán (Yuc), Zacatecas (ZAC).

Categorías de protección en la NOM-059-SEMARNAT-2010: Probablemente extinta en el medio silvestre (E); En peligro de extinción (P); Amenazadas (A); Sujetas a protección especial (Pr).

\begin{tabular}{|c|c|}
\hline Agavaceae & Agave attenuata Salm-Dyck \\
\hline Agave aktites Gentry & Agave aurea Brandegee (BCS) \\
\hline Agave albopilosa I. Cabral, Villareal et A.E. & Agave avellanidens Trel. (BC) \\
\hline Estrada & Agave bovicornuta Gentry \\
\hline * Agave americana L. ssp. protamericana Gentry & Agave bracteosa S. Watson ex Engelm. (A) \\
\hline Agave americana L. var. oaxacensis Gentry & ** Agave cantala Roxb. var. acuispina (Trel.) \\
\hline Agave angustiarum Trel. & Gentry \\
\hline $\begin{array}{l}\text { Agave angustifolia Haw. var. deweyana (Trel.) } \\
\text { Gentry }\end{array}$ & $\begin{array}{l}\text { Agave capensis Gentry (BCS) } \\
\text { Agave cerulata Trel. ssp. cerulata }\end{array}$ \\
\hline $\begin{array}{l}\text { Agave angustifolia Haw. var. rubescens (Salm- } \\
\text { Dyck) Gentry }\end{array}$ & $\begin{array}{l}\text { Agave cerulata Trel. ssp. dentiens (Trel.) Gentry } \\
\text { Agave cerulata Trel. ssp. nelsonii (Trel.) Gentry }\end{array}$ \\
\hline Agave angustifolia Haw. var. sargentii Trel. & (BC) \\
\hline Agave applanata K. Koch ex Jacobi & Agave cerulata Trel. ssp. subcerulata Gentry \\
\hline $\begin{array}{l}\text { Agave arcedianoensis Cházaro, O.M. Valencia et } \\
\text { Vázquez (JAL) }\end{array}$ & $\begin{array}{l}\text { (BCS) } \\
\text { Agave chazaroi Vázquez et O.M. Valencia (JAL) }\end{array}$ \\
\hline Agave asperrima Jacobi ssp. maderensis (Gentry) & Agave chiapensis Jacobi (CHIS) (Pr) \\
\hline B. Ull & Agave chrysoglossa I.M. Johnst. \\
\hline * Agave asperrima Jacobi ssp. asperrima & Agave colimana Gentry \\
\hline $\begin{array}{l}\text { Agave asperrima Jacobi ssp. potosiensis (Gentry) } \\
\text { B. Ullrich }\end{array}$ & $\begin{array}{l}\text { Agave collina Greenm. } \\
\text { Agave colorata Gentry }\end{array}$ \\
\hline perrima Jacobi ssp. zarcensis (Gentry) & gesta Gentry (CHIS) (Pr) \\
\hline B. Ullrich (DGO) & Agave cupreata Trel. et A. Berger \\
\hline $\begin{array}{l}\text { Agave atrovirens Karw. ex Salm-Dyck var. } \\
\text { atrovirens }\end{array}$ & $\begin{array}{l}\text { ** Agave dasylirioides Jacobi et C.D. Bouché (A) } \\
\text { Agave datylio F.A.C. Weber var. datylio (BCS) }\end{array}$ \\
\hline $\begin{array}{l}\text { Agave atrovirens Karw. ex Salm-Dyck var. } \\
\text { mirabilis (Trel.) Gentry }\end{array}$ & $\begin{array}{l}\text { Agave datylio F.A.C. Weber var. vexans (Trel.) } \\
\text { I.M. Johnst. (BCS) }\end{array}$ \\
\hline
\end{tabular}


Apéndice 1. Continuación.

*Agave deserti Engelm. ssp. deserti
* Agave deserti Engelm. ssp. pringlei (Engelm.
ex Baker) Gentry
* Agave deserti Engelm. ssp. simplex Gentry
Agave desmetiana Jacobi (SIN)
Agave difformis A. Berger
Agave durangensis Gentry
Agave ellemeetiana Jacobi (VER)
Agave ensifera Jacobi
Agave felgeri Gentry (SON)
Agave filifera Salm-Dyck ssp. schidigera (Lem.)
B. Ullrich

Agave filifera Salm-Dyck ssp. filifera

Agave filifera Salm-Dyck ssp. microcreps

Kimnach (SIN)

Agave filifera Salm-Dyck ssp. multifilifera

(Gentry) B. Ullrich

Agave flexispina Trel.

Agave fortiflora Gentry (SON)

Agave funkiana K. Koch et Bouché

Agave garcia-mendozae Galván et L. Hern.

Agave geminiflora (Tagl.) Ker Gawl. (NAY)

Agave gentry $\mathrm{B}$. Ullrich

** Agave ghiesbreghtii Jacobi

Agave gigantensis Gentry (BCS)

* Agave glomeruliflora (Engelm.) A. Berger

* Agave gracilipes Trel.

Agave grijalvensis B. Ullrich (CHIS)

Agave guadalajarana Trel.

Agave guiengola Gentry (OAX) (A)

Agave gypsophila Gentry (Pr)

* Agave havardiana Trel.

** Agave hiemiflora Gentry

Agave hookeri Jacobi

Agave horrida Lem. ex Jacobi ssp. horrida

Agave horrida Lem. ex Jacobi ssp. perotensis B. Ullrich

Agave impressa Gentry (A)

Agave inaequidens $\mathrm{K}$. Koch ssp. barrancensis

Gentry (DGO)

Agave inaequidens K. Koch ssp. inaequidens

Agave isthmensis García-Mend. et Palma

Agave jaiboli Gentry

Agave karwinskii Zucc.

Agave kerchovei Lem.

* Agave lechugilla Torr.
* Agave lophantha Schiede

Agave lurida Aiton (P)

Agave macroacantha Zucc.

Agave mapisaga Trel. var. lisa Gentry (QRO)

Agave mapisaga Trel. var. mapisaga

Agave margaritae Brandegee (BCS)

Agave marmorata Roezl

Agave maximiliana Baker var. katharinae (A.

Berger) Gentry

Agave maximiliana Baker var. maximiliana

Agave microceps (Kimnach) A. Vázquez et

Cházaro

Agave mitis Mart. var. albidior (Salm-Dyck) B.

Ullrich (HGO)

Agave mitis Mart. var. mitis

Agave montana Villareal (NL)

Agave montium-sancticaroli García-Mend.

(TAM)

Agave moranii Gentry (BC)

* Agave murpheyi F. Gibson

Agave nayaritensis Gentry (NAY)

* Agave neomexicana Wooton et Standl.

Agave nizandensis Cutak (OAX) (P)

Agave nuusaviorum García-Mend. ssp. deltoidea García-Mend. (OAX)

Agave nuusaviorum García-Mend. ssp. nuusaviorum (OAX)

Agave obscura Schiede ex Schltdl.

Agave ocahui Gentry var. longifolia Gentry

(SON)

Agave ocahui Gentry var. ocahui (SON)

Agave ornithobroma Gentry (Pr)

Agave oroensis Gentry (ZAC)

Agave ovatifolia G.D. Starr et Villareal (NL)

** Agave pachycentra Trel.

* Agave palmeri Engelm.

Agave parrasana A. Berger (Pr)

Agave parryi Engelm. var. huachucensis (Baker)

Little

* Agave parryi Engelm. var. parryi

Agave parryi Engelm. var. truncata Gentry

Agave parviflora Torr. ssp. flexiflora Gentry

(SON) (A)

* Agave parviflora Torr. ssp. parviflora (A)

Agave peacockii Croucher (Pr)

Agave pedunculifera Trel. 
Apéndice 1. Continuación.

Agave pelona Gentry (SON)

Agave pendula Schnittsp.

Agave petrophila García-Mend. et E. Martínez

Agave pintilla S. González, M. González et L.

Reséndiz (DGO)

Agave polianthiflora Gentry (A)

Agave polyacantha Haw.

Agave potatorum Zucc.

Agave potrerana Trel.

Agave promontorii Trel. (BCS)

Agave pumila hort. ex Baker (COAH)

Agave rhodacantha Trel.

Agave rzedowskiana P. Carrillo, Vega et R. Delgad.

Agave salmiana Otto ex Salm-Dyck ssp.

crassispina (Trel.) Gentry

Agave salmiana Otto ex Salm-Dyck var. ferox (K.

Koch) Gentry

Agave salmiana Otto ex Salm-Dyck var. salmiana

Agave scaposa Gentry

Agave schneideriana A. Berger (PUE)

* Agave schottii Engelm. var. schottii

** Agave schottii Engelm. var. treleasei (Toumey)

Kearney et Peebles

Agave sebastiana Greene

** Agave seemanniana Jacobi

Agave shawii Engelm. ssp. goldmaniana (Trel.)

Gentry (BC)

* Agave shawii Engelm. ssp. shawii

Agave shrevei Gentry ssp. magna Gentry

Agave shrevei Gentry ssp. matapensis Gentry

(SON)

Agave shrevei Gentry ssp. shrevei

Agave sobria Brandegee ssp. frailensis Gentry

(BCS)

Agave sobria Brandegee ssp. roseana (Trel.)

Gentry (BCS)

Agave sobria Brandegee ssp. sobria (BCS)

Agave spicata Cav. (HGO)

Agave striata Zucc. ssp. striata

Agave striata Zucc. var. falcata (Engelm.) Gentry

Agave stricta Salm-Dyck

Agave stringens Trel. (JAL)

Agave subsimplex Trel. (SON)

Agave tenuifolia Zamudio et E. Sánchez

Agave tequilana F.A.C. Weber

Agave titanota Gentry (OAX) (Pr)
Agave triangularis Jacobi

Agave turneri R.H. Webb et Salazar-Ceseña (BC)

Agave valenciana Cházaro et A. Vázquez (JAL)

Agave vazquezgarciae Cházaro et J.A. Lomelí

(JAL)

Agave victoriae-reginae T. Moore (P)

Agave vilmoriniana A. Berger

Agave vizcainoensis Gentry (BCS) (Pr)

* Agave weberi Cels ex Poisson

Agave wendtii Cházaro (VER)

Agave wocomahi Gentry

Agave xylonacantha Salm-Dyck

Agave zebra Gentry (SON)

** Beschorneria albiflora Matuda (Pr)

Beschorneria calcicola García-Mend. (Pr)

* Beschorneria dubia Carrièrre

Beschorneria rigida Rose

Beschorneria septentrionalis García-Mend.

Beschorneria tubiflora (Kunth et Bouché) Kunth

(Pr)

Beschorneria wrightii Hook. (MÉX) (Pr)

Beschorneria yuccoides K. Koch var. dekosteriana (K. Koch) García-Mend.

Beschorneria yuccoides K. Koch var. yuccoides (HGO)

Furcraea cahum Trel.

** Furcraea guatemalensis Trel.

Furcraea guerrerensis Matuda

Furcraea longaeva Karw. et Zucc.

Furcraea macdougallii Matuda (E)

Furcraea martinezii García-Mend. et L. de la

Rosa (GRO)

Furcraea niquivilensis Matuda ex García-Mend.

(CHIS)

Furcraea parmentieri (Roezl ex Ortgies) GarcíaMend. (A)

** Furcraea quicheensis Trel.

** Furcraea samalana Trel.

Furcraea selloa K. Koch (HGO)

Furcraea undulata Jacobi

Hesperaloe campanulata G.D. Starr (NL)

Hesperaloe chiangii (G.D. Starr) B.L. Turner

(SLP)

* Hesperaloe funifera (K. Koch) Trel.

Hesperaloe malacophylla Hochstätter et Mart.Aval. (TAM) 
Apéndice 1. Continuación.

\begin{tabular}{l}
\hline Hesperaloe nocturna Gentry (SON) \\
Hesperaloe parviflora (Torr.) J.M. Coult. ssp. \\
betcholdii Hochstätter (COAH) \\
* Hesperaloe parviflora (Torr.) J.M. Coult. ssp. \\
parviflora \\
Hesperaloe tenuifolia G.D. Starr (SON) \\
Hesperoyucca whipplei Torr. ssp. eremica Epling \\
et A.L. Haines \\
Hesperoyucca whipplei Torr. ssp. rigata Afferni \\
et Drovandi (BC) \\
Manfreda brunnea (S. Watson) Rose (A) \\
Manfreda bulbulifera Castillejos et E. Solano \\
(GRO)
\end{tabular}

Manfreda chamelensis Lott et Verh.-Will. (JAL)

Manfreda elongata Rose

Manfreda galvaniae A. Castañeda, S. Franco et García-Mend. (MÉX)

Manfreda guerrerensis Matuda (GRO) (Pr)

Manfreda guttata (Jacobi et Bouché) Rose

Manfreda hauniensis (J. Petersen) Verh.-Will.

Manfreda involuta McVaugh (JAL)

Manfreda jaliscana Rose

Manfreda littoralis García-Mend., A. Castañeda et S. Franco

Manfreda longibracteata Verh.-Will.

* Manfreda longiflora (Rose) Verh.-Will. (A)

Manfreda maculata (Mart.) Rose

* Manfreda maculosa (Hook.) Rose

Manfreda malinaltenangensis Matuda (MÉX)

Manfreda nanchichitlensis Matuda (MÉX) (A)

Manfreda paniculata L.Hern., R.A. Orellana et Carnevali

Manfreda parva Aarón Rodr. (GRO)

Manfreda petskinil R.A. Orellana, L. Hern. et Carnevali (YUC)

Manfreda planifolia (S. Watson) Rose (Pr)

Manfreda potosina (B.L. Rob. et Greenm.) Rose (Pr)

Manfreda pringlei Rose

Manfreda pubescens (Regel et Ortgies) Verh.Will. ex Piña

Manfreda revoluta (Klotzsch) Rose (MÉX)

Manfreda rubescens Rose

** Manfreda scabra (Ortega) McVaugh

* Manfreda sileri Verh.-Will.

Manfreda singuliflora (S. Watson) Rose
* Manfreda variegata (Jacobi) Rose

Polianthes bicolor E. Solano et García-Mend. (OAX)

Polianthes densiflora (B.L. Rob. et Fernald) Shinners (CHIH) (Pr)

Polianthes durangensis Rose

Polianthes elongata Rose (GRO)

Polianthes geminiflora (La Llave) Rose var. clivicola McVaugh

Polianthes geminiflora (La Llave) Rose var. geminiflora

Polianthes geminiflora (La Llave) Rose var. graminifolia (Rose) McVaugh

Polianthes geminiflora (La Llave) Rose var. pueblensis E. Solano et García-Mend. (PUE)

Polianthes howardii Verh.-Will. (Pr)

Polianthes longiflora Rose (Pr)

Polianthes montana Rose (NAY)

Polianthes multicolor E. Solano et Dávila (GTO)

Polianthes nelsonii Rose (DGO)

Polianthes oaxacana García-Mend. et E. Solano (OAX)

Polianthes palustris Rose (Pr)

Polianthes platyphylla Rose (Pr)

Polianthes pringlei Rose

Polianthes sessiliflora (Hemsl.) Rose

Polianthes zapopanensis E. Solano et RíosGómez (JAL)

Prochnyanthes mexicana (Zucc.) Rose

Yucca capensis L.W. Lenz (BCS)

* Yucca carnerosana (Trel.) McKelvey

Yucca coahuilensis Matuda et Piña (COAH)

Yucca decipiens Trel.

Yucca declinata Laferr. (SON)

Yucca desmettiana Baker (CHIH)

* Yucca elata (Engelm.) Engelm.

** Yucca elephantipes Regel

Yucca endlichiana Trel. (COAH) (Pr)

* Yucca faxoniana (Trel.) Sarg.

* Yucca filifera Chabaud

Yucca grandiflora Gentry (SON) (Pr)

Yucca jaliscensis (Trel.) Trel.

Yucca lacandonica Gómez Pompa et J. Valdés (A)

Yucca linearifolia Clary

* Yucca madrensis Gentry

Yucca mixtecana García-Mend. 
Apéndice 1. Continuación.

\footnotetext{
Yucca periculosa Baker

Yucca potosina Rzed. (SLP)

Yucca queretaroensis Piña (A)

* Yucca reverchonii Trel.

Yucca rigida (Engelm.) Trel.

* Yucca rostrata Engelm. ex Trel.

* Yucca rupicola Scheele

* Yucca schottii Engelm.

* Yucca thompsoniana Trel.

* Yucca torreyi Shafer

* Yucca treculeana Carrière

Yucca valida Brandegee
}

\section{Alismaceae}

Echinodorus virgatus (Hook. et Arn.) Micheli (NAY) (E)

Sagittaria demersa J.G. Sm.

Sagittaria macrophylla Zucc. (A)

\author{
Alliaceae \\ Allium durangoense Traub \\ Allium eurotophilum Wiggins (BC) \\ Allium fantasmosense Traub (SLP) \\ *** Allium glandulosum Link et Otto \\ Allium hintoniorum B.L. Turner (NL) \\ Allium huntiae Traub (DGO) \\ Allium kunthii G. Don \\ Allium mannii Traub et T.M. Howard (DGO) \\ Allium melliferum Traub (JAL) \\ Allium mexicanum Traub (TAM) \\ Allium michoacanum Traub (MICH) \\ Allium ownbeyi Traub (NL) \\ Allium peninsulare Lemmon ex Greene \\ * Allium plummerae S. Watson \\ Allium potosiense Traub (SLP) \\ Allium pueblanum Traub (PUE) \\ * Allium runyonii Ownbey \\ Allium stoloniferum T.D. Jacobsen \\ Allium telaponense Traub (MÉX) \\ Allium traubii T.M. Howard (NL) \\ Bessera elegans Schult.f. \\ Bessera tenuiflora (Greene) J.F. MacBr. (BCS) \\ Dandya balsensis López-Ferrari et Espejo \\ Dandya hannibalii L.W. Lenz (MICH) \\ Dandya purpusii (Brandegee) H.E. Moore \\ (COAH)
}

Dandya thadhowardii L.W. Lenz

Diphalangium graminifolium S. Schauer (VER)

Jaimehintonia gypsophila B.L. Turner (NL)

*** Milla biflora Cav.

Milla bryanii I.M. Johnst. (COAH)

Milla delicata H.E. Moore (GRO)

Milla filifolia T.M. Howard

Milla magnifica H.E. Moore

Milla mexicana T.M. Howard

Milla mortoniana H.E. Moore (GRO)

Milla oaxacana Ravenna (OAX)

Milla potosina T.M. Howard

Milla rosea H.E. Moore (NL)

Petronymphe decora H.E. Moore (GRO) (P)

Triteleia guadalupensis L.W. Lenz (BC)

Triteleiopsis palmeri (S. Watson) Hoover (BC)

\section{Alstroemeriaceae}

Bomarea acutifolia (Link et Otto) Herb.

Bomarea gloriosa (Schltdl. et Cham.) M. Roem.

Amaryllidaceae

Chlidanthus ehrenbergii (Klotzsch) Kunth (OAX)

Habranthus arenicola (Brandegee) Flagg, G.

Lom. Sm. \& Meerow (BC)

Habranthus chichimeca (T.M. Howard et Ogden)

Flagg, G. Lom. Sm. \& Meerow

Habranthus conzattii (Greenm.) Flagg, G. Lom.

Sm. \& Meerow (OAX)(A)

* Habranthus longifolia (Hemsl.) Flagg, G. Lom.

Sm. \& Meerow (SLP)

Habranthus medinae L.O. Alvarado et García-

Mend. (PUE)

Habranthus mexicanus T.M. Howard

Habranthus tepicensis Greenm. ex Flagg et G.

Lom. Sm. (NAY)

Habranthus vittatus T.M. Howard (OAX)

Hymenocallis acutifolia (Herb.) Sweet

Hymenocallis araniflora T.M. Howard

Hymenocallis astrostephana T.M. Howard

(GRO)

Hymenocallis azteciana Traub

Hymenocallis baumlii Ravenna (CHIS)

Hymenocallis cleo Ravenna (CHIS)

Hymenocallis clivorum Laferr. (SON) 
Apéndice 1. Continuación.

Hymenocallis concinna Baker (P)

Hymenocallis cordifolia Micheli (GRO)

Hymenocallis durangoensis T.M. Howard (DGO)

(P)

Hymenocallis eucharidifolia Baker (GRO)

Hymenocallis glauca (Herb.) Baker ex Benth. et

Hook.f.

Hymenocallis graminifolia Greenm. (MOR)

Hymenocallis guerreroensis T.M. Howard

(GRO) (A)

Hymenocallis harrisiana Herb.

Hymenocallis howardii Bauml

Hymenocallis imperialis T.M. Howard

Hymenocallis jaliscensis M.E. Jones

Hymenocallis leavenworthii (Standl. et Steyerm.)

Bauml (MICH) (A)

Hymenocallis lehmilleri T.M. Howard (GRO)

Hymenocallis longibracteata Hochr. (VER)

Hymenocallis maximilianii T.M. Howard (GRO)

Hymenocallis partita Ravenna (CHIS)

Hymenocallis phalangidis Bauml (NAY)

Hymenocallis pimana Laferr. (CHIH)

Hymenocallis portamonetensis Ravenna (CHIS)

Hymenocallis proterantha $\mathrm{Bauml}$

Hymenocallis pumila Bauml

Hymenocallis sonorensis Standl.

Hymenocallis vasconcelosii García-Mend. (OAX)

Hymenocallis woelfleana T.M. Howard

** Sprekelia formosissima (L.) Herb.

Sprekelia howardii Lehmiller

Zephyranthes bella T.M. Howard et Ogden

** Zephyranthes brevipes (Baker ex Donn. Sm.)

Standl.

** Zephyranthes chlorosolen (Herb.) D. Dietr.

** Zephyranthes chrysantha Greenm. et C.H.

Thomps.

Zephyranthes clintiae Traub

Zephyranthes concolor (Lind1.) Benth. et Hook. f.
Zephyranthes crociflora T.M. Howard et Ogden

\section{(COAH)}

Zephyranthes dichromantha T.M. Howard (SLP)

** Zephyranthes drummondii D. Don

** Zephyranthes erubescens S. Watson

Zephyranthes fosteri Traub

Zephyranthes howardii Traub

Zephyranthes katheriniae Spencer (HGO)
Zephyranthes latissimifolia Spencer (JAL)

Zephyranthes leucantha T.M. Howard (HGO)

Zephyranthes longituba Flory ex Flagg et G.

Lom.Sm.

Zephyranthes macrosiphon Baker

Zephyranthes minuta (Kunth) D. Dietr.

Zephyranthes miradorensis (Kraenzl.) Espejo et

López-Ferr. (VER)

Zephyranthes moctezumae T.M. Howard (SLP)

Zephyranthes morrisclintii Traub et T.M. Howard (NL)

Zephyranthes nelsonii Greenm.

Zephyranthes nymphaea T.M. Howard et Ogden

Zephyranthes orellanae Carnevali, Duno et J.L.

Tapia (YUC)

Zephyranthes primulina T.M. Howard et Ogden

(SLP)

Zephyranthes reginae T.M. Howard et Ogden

(SLP)

** Zephyranthes sessilis Herb.

Zephyranthes subflava Spencer (SLP)

Anthericaceae

Echeandia albiflora (Schltdl. et Cham.) M.

Martens et Galeotti (VER)

Echeandia atoyacana Cruden

Echeandia attenuata Cruden

Echeandia breedlovei Cruden

Echeandia campechiana Cruden

* Echeandia chandleri (Greenm. et C.H.

Thomps.) Cruden

Echeandia chiapensis Cruden

Echeandia coalcomanensis Cruden (MICH)

Echeandia confertiflora Cruden (OAX)

Echeandia conzattii Cruden (OAX)

Echeandia drepanoides (Greenm.) Cruden

(OAX)

Echeandia durangensis (Greenm.) Cruden

Echeandia echeandioides (Schltdl.) Cruden

Echeandia elegans Cruden

Echeandia falcata Cruden

* Echeandia flavescens (Schult. et Schult.f.)

Cruden

Echeandia flexuosa Greenm.

Echeandia gentryi Cruden

Echeandia gracilis Cruden 
Apéndice 1. Continuación.

\begin{tabular}{|c|c|}
\hline \multirow{2}{*}{\multicolumn{2}{|c|}{$\begin{array}{l}\text { Echeandia grandiflora Cruden (OAX) } \\
\text { Echeandia hallbergii Cruden (OAX) }\end{array}$}} \\
\hline & \\
\hline Echeandia hintonii Cruden (GRO) & Araceae \\
\hline Echeandia hirticaulis Cruden & Anthurium andicola Liebm. \\
\hline Echeandia imbricata Cruden & ** Anthurium armeniense Croat \\
\hline Echeandia llanicola Cruden (OAX) & Anthurium berriozabalenze Matuda \\
\hline Echeandia longifolia (Weath.) Cruden & Anthurium cerrobaulense Matuda \\
\hline ** Echeandia longipedicellata Cruden & Anthurium cerropelonense Matuda (OAX) \\
\hline ** Echeandia luteola Cruden & Anthurium chamulense Matuda ssp. chamulense \\
\hline Echeandia macrophylla Rose ex Weath. (SLP) & (CHIS) \\
\hline Echeandia mcvaughii Cruden & Anthurium chamulense Matuda ssp. oaxacanum \\
\hline Echeandia magnifica López-Ferr., Espejo et Ceja & Croat $(\mathbf{O A X})$ \\
\hline (GRO) & ** Anthurium chiapasense Standl. ssp. \\
\hline ** Echeandia matudae Cruden & chiapasense \\
\hline Echeandia mexiae Cruden & Anthurium chiapasense Standl. ssp. tlaxiacense \\
\hline Echeandia mexicana Cruden & (Matuda) Croat (OAX) \\
\hline $\begin{array}{l}\text { Echeandia michoacensis (Poelln.) Cruden } \\
\text { (MICH) }\end{array}$ & $\begin{array}{l}\text { Anthurium clarinervium Matuda (CHIS) } \\
\text { ** Anthurium cordatotriangylum Matuda }\end{array}$ \\
\hline Echeandia mirandae Cruden & Anthurium faustomirandae Pérez-Far. et Croat \\
\hline Echeandia montealbanensis Cruden (OAX) & (CHIS) \\
\hline Echeandia nana (Baker) Cruden & ** Anthurium flexile Schott ssp. muelleri (J.F. \\
\hline Echeandia nayaritensis Cruden & MacBr.) Croat et R.A. Baker \\
\hline Echeandia oaxacana Cruden (OAX) & Anthurium hagsaterianum J.R. Haager (GRO) \\
\hline Echeandia occidentalis Cruden & Anthurium halmoorei Croat \\
\hline Echeandia palmeri Cruden & Anthurium huautlense Matuda (OAX) \\
\hline Echeandia paniculata Rose & ** Anthurium huixtlense Matuda \\
\hline Echeandia parva Cruden & Anthurium leuconeurum Lem. (CHIS) \\
\hline Echeandia parvicapsulata Cruden & Anthurium lezamae Matuda \\
\hline ** Echeandia parviflora Baker & Anthurium longipeltatum Matuda \\
\hline Echeandia pihuamensis Cruden (JAL) & ** Anthurium lucens Standl. \\
\hline Echeandia platyphylla (Greenm.) Cruden (PUE) & Anthurium machetioides Matuda \\
\hline Echeandia pseudopetiolata Cruden (GRO) & ** Anthurium montanum Hemsl. \\
\hline Echeandia pseudoreflexa Cruden (CHIS) & Anthurium nakamurae Matuda (CHIS) \\
\hline Echeandia ramosissima (C. Presl) Cruden & Anthurium nelsonii Croat (OAX) \\
\hline * Echeandia reflexa (Cav.) Rose & Anthurium nizandense Matuda \\
\hline Echeandia robusta Cruden & Anthurium ocotepecense Matuda \\
\hline Echeandia sanmiguelensis Cruden (GTO) & Anthurium pedatoradiatum Schott ssp. \\
\hline Echeandia scabrella (Benth.) Cruden & helleborifolium (Schott) Croat \\
\hline Echeandia sinaloensis Cruden & Anthurium pedatoradiatum Schott ssp. \\
\hline Echeandia smithii Cruden (OAX) & pedatoradiatum \\
\hline Echeandia tamaulipensis Cruden (TAM) & Anthurium podophyllum (Cham. et Schltdl.) \\
\hline Echeandia taxcana Cruden & Kunth (A) \\
\hline Echeandia tenuifolia Cruden (OAX) & Anthurium riograndicolum Matuda (OAX) \\
\hline Echeandia tenuis (Weath.) Cruden (GRO) & Anthurium rionegrense Matuda \\
\hline Echeandia udipratensis Cruden (JAL) & Anthurium rzedowskii Croat \\
\hline Echeandia vaginata Cruden (OAX) & Anthurium sarukhanianum Croat et Haager (GRO) \\
\hline
\end{tabular}


Apéndice 1. Continuación.

\begin{tabular}{|c|c|}
\hline Anthurium schlechtendalii Kunth ssp. jimenezii & Brahea aculeata (Brandegee) H.E. Moore (A) \\
\hline ** Anthurium seleri Engl. & Brahea berlandieri Bartlett (Pr) \\
\hline Anthurium subovatum Matuda (OAX) & Brahea brandegeei (Purpus) H.E. Moore \\
\hline ** Anthurium titanium Standl. et Steyerm. & Brahea calcarea Liebm. (VER) \\
\hline Anthurium umbrosum Liebm. & Brahea decumbens Rzed. \\
\hline ** Anthurium verapazense Engl. & ** Brahea dulcis (Kunth) Mart. \\
\hline Anthurium yetlense Matuda (OAX) & Brahea edulis H. Wendl. ex S. Watson (BC)(Pr) \\
\hline $\begin{array}{l}\text { Arisaema macrospathum Benth. } \\
\text { ** Monstera acacoyaguensis Matuda }\end{array}$ & $\begin{array}{l}\text { Brahea elegans (Franceschi ex Becc.) H.E. Moore } \\
\text { (SON) }\end{array}$ \\
\hline $\begin{array}{l}\text { Monstera florescanoana Croat, Krömer et A. } \\
\text { Acebey (VER) }\end{array}$ & $\begin{array}{l}\text { Brahea moorei L.H. Bailey (Pr) } \\
\text { Brahea nitida André (Pr) }\end{array}$ \\
\hline ** Philodendron advena Schott & Brahea pimo Becc. \\
\hline Philodendron bazii Matuda & ** Brahea salvadorensis $\mathrm{H}$. Wendl. ex Becc. \\
\hline Philodendron breedlovei Croat (CHIS) & Brahea sarukhanii H.J. Quero \\
\hline Philodendron dressleri G.S. Bunting & Chamaedorea affinis Liebm. ex Mart. (OAX) \\
\hline Philodendron escuintlense Matuda & Chamaedorea alternans $\mathrm{H}$. Wendl. (A) \\
\hline $\begin{array}{l}\text { Philodendron hederaceum (Willd.) Schott et } \\
\text { Endl. var. oxycardium (Schott) Croat }\end{array}$ & $\begin{array}{l}\text { ** Chamaedorea arenbergiana } \mathrm{H} \text {. Wendl. (A) } \\
\text { Chamaedorea atrovirens Mart. (OAX) (A) }\end{array}$ \\
\hline ** Philodendron purulhense Croat & ** Chamaedorea carchensis Standl. et Steyerm. \\
\hline $\begin{array}{l}\text { Philodendron radiatum Schott var. } \\
\text { pseudoradiatum (Matuda) Croat (CHIS) }\end{array}$ & $\begin{array}{l}\text { (A) } \\
\text { Chamaedorea cataractarum Mart. (A) }\end{array}$ \\
\hline Philodendron seguine Schott & ** Chamaedorea elatior Mart. (A) \\
\hline ** Philodendron smithii Engl. & ** Chamaedorea elegans Mart. \\
\hline Philodendron sousae Croat (CHIS) & ** Chamaedorea ernesti-augusti $\mathrm{H}$. Wendl. (A) \\
\hline $\begin{array}{l}\text { Philodendron subincisum Schott (VER) } \\
\text { ** Philodendron verapazense Croat }\end{array}$ & $\begin{array}{l}\text { ** Chamaedorea fractiflexa Hodel et J.J. Castillo } \\
\text { (A) }\end{array}$ \\
\hline ** Spathiphyllum blandum Schott & Chamaedorea glaucifolia H. Wendl. (CHIS) (P) \\
\hline Spathiphyllum brevirostre (Liebm.) Schott & Chamaedorea hooperiana Hodel (VER) (A) \\
\hline ** Spathiphyllum cochlearispathum (Liebm.) & Chamaedorea ibarrae Hodel \\
\hline Engl. & ** Chamaedorea keeleriorum Hodel et J.J. \\
\hline ** Spathiphyllum ortgiesii Regel & Castillo \\
\hline Spathiphyllum uxpanapense Matuda (VER) & Chamaedorea klotzschiana H. Wendl. (Pr) \\
\hline ** Syngonium chiapense Matuda & Chamaedorea liebmannii Mart. (A) \\
\hline ** Syngonium neglectum Schott & Chamaedorea martiana $\mathrm{H}$. Wendl. (CHIS) \\
\hline $\begin{array}{l}\text { Syngonium sagittatum G.S. Bunting } \\
* * \text { Syngonium salvadorense Schott }\end{array}$ & $\begin{array}{l}\text { Chamaedorea metallica O.F. Cook ex H.E. } \\
\text { Moore (P) }\end{array}$ \\
\hline ** Syngonium steyermarkii Croat & Chamaedorea microspadix Burret (A) \\
\hline Xanthosoma mendozae Matuda & ** Chamaedorea neurochlamys Burret \\
\hline Xanthosoma yucatanense Engl. & $\begin{array}{l}\text { ** Chamaedorea nubium Standl. et Steyerm. (A) } \\
\text { ** Chamaedorea oblongata Mart. }\end{array}$ \\
\hline Arecaceae & Chamaedorea oreophila Mart. (A) \\
\hline ** Astrocaryum mexicanum Liebm. ex Mart. & ** Chamaedorea parvisecta Burret (A) \\
\hline Attalea guacuyule (Liebm. ex Mart.) Zona & Chamaedorea plumosa Hodel (CHIS) \\
\hline $\begin{array}{l}\text { ** Bactris mexicana Liebm. ex Mart. var. } \\
\text { trichophylla Burret }\end{array}$ & $\begin{array}{l}\text { Chamaedorea pochutlensis Liebm. (A) } \\
\text { Chamaedorea pringlei } \mathrm{S} \text {. Watson }\end{array}$ \\
\hline
\end{tabular}


Apéndice 1. Continuación.

Chamaedorea queroana Hodel (OAX) (A)

Chamaedorea radicalis Mart.

Chamaedorea rhizomatosa Hodel (OAX) (A)

Chamaedorea rigida $\mathrm{H}$. Wendl. ex Dammer

(OAX) (A)

** Chamaedorea rojasiana Standl. et Steyerm.

(A)

** Chamaedorea sartorii Liebm. (A)

Chamaedorea schiedeana Mart. (A)

** Chamaedorea schippii Burret

** Chamaedorea seifrizii Burret

** Chamaedorea simplex Burret (A)

Chamaedorea stolonifera $\mathrm{H}$. Wendl. ex Hook.f.

(A)

** Chamaedorea tuerckheimii (Dammer) Burret

(P)

** Chamaedorea vulgata Standl. et Steyerm. (A)

** Chamaedorea whitelockiana Hodel et N.W.

Uhl (A)

Coccothrinax readii $\mathrm{H}$.J. Quero (A)

Cryosophila nana (Kunth) Blume ex Salomon (A)

** Cryosophila stauracantha (Heynh.) R.J. Evans (A)

** Desmoncus ferox Bartlett

** Desmoncus quasillarius Bartlett

Gaussia gomez-pompae (H.J. Quero) H.J. Quero

(A)

** Gaussia maya (O.F. Cook) H.J. Quero et Read

(A)

** Reinhardtia elegans Liebm. (A)

** Roystonea dunlapiana P.H. Allen (Pr)

Sabal gretherae H.J. Quero (QROO) (Pr)

*** Sabal mexicana Mart.

Sabal pumos (Kunth) Burret (Pr)

Sabal rosei (O.F. Cook) Becc.

Sabal uresana Trel. (Pr)

\section{Bromeliaceae}

Aechmea aenigmatica López-Ferr., Espejo, Ceja et Mendoza (OAX)

** Aechmea matudae L.B. Sm.

Aechmea mexicana Baker

** Billbergia pallidiflora Liebm.

** Billbergia viridiflora $\mathrm{H}$. Wendl.

Bromelia hemisphaerica Lam.

Bromelia palmeri $\mathrm{Mez}$
Catopsis compacta $\mathrm{Mez}$

** Catopsis delicatula L.B. Sm.

Catopsis mexicana L.B. Sm.

Catopsis minimiflora Matuda (CHIS)

** Catopsis subulata L.B. Sm.

Catopsis wawranea Mez (VER)

** Fosterella micrantha (Lind1.) L.B. Sm.

Greigia juareziana L.B. Sm.

Greigia van-hyningii L.B. Sm.

Hechtia bracteata Mez (VER)

Hechtia carlsoniae Burt-Utley et Utley (GRO)

Hechtia caudata L.B. Sm. (OAX)

Hechtia caulescens López-Ferr., Espejo et Mart.-

Correa

Hechtia chichinautzensis Mart.-Correa, Espejo et

López-Ferr. (MOR)

Hechtia colossa Mart.-Correa, Espejo et López-

Ferr. (OAX)

Hechtia confusa L.B. Sm. (PUE)

Hechtia conzattiana L.B. Sm. (OAX)

Hechtia edulis I. Ramírez, Espejo et López-Ferr.

(CHIH)

Hechtia elliptica L.B. Sm. (COAH)

Hechtia epigyna Harms

Hechtia fosteriana L.B. Sm. (OAX)

Hechtia fragilis Burt-Utley et Utley

Hechtia galeottii Mez (OAX)

Hechtia gayorum L.W. Lenz (BCS)

Hechtia ghiesbreghtii Lem. (CHIS)

Hechtia glabra Brandegee (VER)

Hechtia glauca Burt-Utley et Utley (MICH)

Hechtia glomerata Zucc.

** Hechtia guatemalensis Mez

Hechtia hintoniana Burt-Utley, Utley et GarcíaMend. (MÉX)

Hechtia iltisii Burt-Utley et Utley (JAL)

Hechtia jaliscana L.B. Sm. (JAL)

Hechtia laevis L.B. Sm. (COL)

Hechtia lanata L.B. Sm. (OAX)

Hechtia laxissima L.B. Sm. (MICH)

Hechtia lundelliorum L.B. Sm.

Hechtia lyman-smithii Burt-Utley et Utley (OAX)

Hechtia marnier-lapostollei L.B. Sm. (OAX)

Hechtia matudae L.B. Sm.

Hechtia melanocarpa L.B. Sm. (GRO)

Hechtia mexicana L.B. Sm. (SLP) 
Apéndice 1. Continuación.

\author{
Hechtia michoacana Burt-Utley, Utley et García- \\ Mend. (MICH) \\ Hechtia montana Brandegee \\ Hechtia mooreana L.B. Sm. (GRO) \\ Hechtia myriantha Mez (VER) \\ Hechtia nuusaviorum Espejo et López-Ferr. \\ (OAX) \\ Hechtia oaxacana Burt-Utley, Utley et García- \\ Mend. (OAX) \\ Hechtia pedicellata S. Watson (JAL)
}

Hechtia perotensis I. Ramírez et Mart.-Correa

Hechtia podantha Mez

Hechtia pretiosa Espejo et López-Ferr. (GTO)

Hechtia pringlei B.L. Rob. et Greenm. (OAX)

Hechtia pumila Burt-Utley et Utley (GRO)

Hechtia purpusii Brandegee (VER)

Hechtia reflexa L.B. Sm.

Hechtia reticulata L.B. Sm.

Hechtia rosea E. Morren ex Baker

Hechtia roseana L.B. Sm.

** Hechtia schottii Baker

Hechtia sphaeroblasta B.L. Rob.

Hechtia stenopetala Klotzsch (VER)

Hechtia suaveolens E. Morren ex Mez

Hechtia subalata L.B. Sm. (DGO)

Hechtia tehuacana B.L. Rob. (PUE)

* Hechtia texensis S. Watson

Hechtia tillandsioides (André) L.B. Sm.

Hechtia zamudioi Espejo, López-Ferr. et I.

Ramírez (QRO)

Hohenbergia mesoamericana I. Ramírez,

Carnevali et Cetzal (QROO)

** Hohenbergiopsis guatemalensis (L.B. Sm.)

L.B. Sm. et Read

Pepinia amblyosperma (L.B. Sm.) G.S. Varad. et Gilmartin

** Pepinia punicea (Scheidw.) Brongn. ex André

Pitcairnia abundans L.B. Sm. (NAY)

Pitcairnia breedlovei L.B. Sm. (CHIS)

** Pitcairnia calderonii Standl. et L.B. Sm.

** Pitcairnia carioana Wittm.

** Pitcairnia chiapensis Miranda

Pitcairnia colimensis L.B. Sm.

Pitcairnia compostelae McVaugh

Pitcairnia cylindrostachya L.B. Sm.

Pitcairnia densiflora Brongn. ex Lem.
Pitcairnia flavescentia Matuda (GRO)

Pitcairnia flexuosa L.B. Sm.

Pitcairnia foliacea L.B. Sm. (MICH)

Pitcairnia hintoniana L.B. Sm.

Pitcairnia jaliscana S. Watson (JAL)

Pitcairnia karwinskyana Schult. et Schult.f.

Pitcairnia leprosa L.B. Sm. (GRO)

Pitcairnia loki-schmidtii Rauh et Barthlott (JAL)

Pitcairnia matudae L.B. Sm. (CHIS)

Pitcairnia micheliana André

Pitcairnia micropoda L.B. Sm. (MÉX)

Pitcairnia mirandae Utley et Burt-Utley (CHIS)

Pitcairnia modesta L.B. Sm. (GRO)

Pitcairnia monticola Brandegee (SIN)

Pitcairnia mooreana L.B. Sm. (GRO)

Pitcairnia oaxacana L.B. Sm.

Pitcairnia ocotensis Beutelsp. et López Vel. (CHIS)

Pitcairnia palmeri S. Watson

Pitcairnia pteropoda L.B. Sm. (MICH)

** Pitcairnia puberula Mez et Donn. Sm.

** Pitcairnia recurvata (Scheidw.) K. Koch

Pitcairnia ringens Klotzsch

Pitcairnia roseana L.B. Sm.

Pitcairnia schiedeana Baker

Pitcairnia secundiflora L.B. Sm. (CHIS)

Pitcairnia sordida L.B. Sm. (GRO)

Pitcairnia tabuliformis Linden (CHIS)

Pitcairnia tillandsioides L.B. Sm. (GRO)

** Pitcairnia tuerckheimii Donn. Sm.

Pitcairnia undulata (hort. ex Beer) Schiedw.

Pitcairnia undulatosepala Rauh (OAX)

Pitcairnia vallisoletana Lex.

Pitcairnia virginalis Utley et Burt-Utley (OAX)

Pitcairnia xanthocalyx Mart.

Pitcairnia yocupitziae Espejo et López-Ferr. (GRO)

Racinaea adscendens (L.B. Sm.) M.A. Spencer et L.B. Sm.

** Racinaea ghiesbreghtii (Baker) M.A. Spencer et L.B. Sm.

Tillandsia achyrostachys E. Morren ex Baker var. achyrostachys

Tillandsia achyrostachys E. Morren ex Baker var. stenolepis L.B. Sm.

Tillandsia aesii I. Ramírez et Carnevali 
Apéndice 1. Continuación.

Tillandsia aguascalientensis C.S. Gardner (AGS)
Tillandsia albida Mez et Purpus
Tillandsia alfredo-lauii Rauh et J. Lehm. (HGO)
Tillandsia alvareziae Rauh (VER)
Tillandsia andrieuxii (Mez) L.B. Sm.
Tillandsia arroyoensis (W. Weber et Ehlers)
$\quad$ Espejo et López-Ferr.
Tillandsia atenangoensis Ehlers et Wulfinghoff
$\quad(\mathbf{O A X )}$

Tillandsia atroviolacea Ehlers et Koide (OAX)

* Tillandsia baileyi Rose ex Small

Tillandsia belloensis W. Weber

Tillandsia beutelspacheri Matuda (CHIS)

Tillandsia bochilensis Ehlers (CHIS)

Tillandsia boqueronensis Ehlers (OAX)

Tillandsia borealis López-Ferr. et Espejo

Tillandsia botterii E. Morren ex Baker (VER)

Tillandsia bourgaei Baker

Tillandsia caballosensis Ehlers (GRO)

Tillandsia califanii Rauh

Tillandsia callichroma L. Hrom. (OAX)

Tillandsia calothyrsus Mez

Tillandsia capistranoensis Ehlers et W. Weber

Tillandsia carlos-hankii Matuda (OAX) (A)

Tillandsia carlsoniae L.B. Sm. (CHIS)

Tillandsia celata Ehlers et Lautner (OAX)

Tillandsia chaetophylla Mez

Tillandsia chapalillaensis Ehlers et Lautner

(NAY)

Tillandsia chiapensis C.S. Gardner (CHIS) (A)

** Tillandsia chlorophylla L.B. Sm.

Tillandsia circinnatioides Matuda

Tillandsia coalcomanensis Ehlers (MICH)

Tillandsia comitanensis Ehlers (CHIS)

Tillandsia concolor L.B. Sm. (A)

Tillandsia copalaensis Ehlers (OAX)

Tillandsia cossonii Baker

Tillandsia cretacea L.B. Sm.

Tillandsia crista-galli Ehlers (CHIS)

Tillandsia cryptantha Baker

** Tillandsia cucaensis Wittm.

Tillandsia X cuchnichim R. Guess et V. Guess

(CHIS)

** Tillandsia dasyliriifolia Baker

** Tillandsia deflexa L.B. Sm.

Tillandsia delicata Ehlers (OAX)
Tillandsia deppeana Steud.

Tillandsia diguetii Mez et Rol.-Goss.

Tillandsia dugesii Baker

Tillandsia durangensis Rauh et Ehlers (DGO)

Tillandsia ehlersiana Rauh (CHIS) (A)

Tillandsia eistetteri Ehlers

** Tillandsia eizii L.B. Sm.

Tillandsia elizabethae Rauh

Tillandsia elusiva Pinzón, I. Ramírez et

Carnevali (CHIS)

Tillandsia erubescens Schltdl.

Tillandsia escahuascensis Espejo, López-Ferr.,

Ceja et A. Mend. (PUE)

Tillandsia exserta Fernald

Tillandsia fascifolia Flores Cruz et Diego-Esc.

(GRO)

Tillandsia ferrisiana L.B. Sm.

** Tillandsia flabellata Baker

Tillandsia flavobracteata Matuda (VER)

Tillandsia floresensis Ehlers (CHIS)

Tillandsia foliosa M. Martens et Galeotti

Tillandsia fresnilloensis W. Weber et Ehlers

Tillandsia fuchsii $\mathrm{W}$. Till var. fuchsii

Tillandsia fuchsii $\mathrm{W}$. Till var. stephani $\mathrm{W}$. Till

Tillandsia glabrior (L.B. Sm.) López-Ferr.,

Espejo et I. Ramírez (OAX)

Tillandsia gracillima L.B. Sm. (PUE)

Tillandsia graebeneri Mez (VER)

** Tillandsia grandis Schltdl.

Tillandsia grandispica Ehlers (GRO)

Tillandsia grossispicata Espejo, López-Ferr. et

W. Till

** Tillandsia guatemalensis L.B. Sm.

Tillandsia guenther-nolleri Ehlers

Tillandsia guerreroensis Rauh (GRO)

Tillandsia gymnobotrya Baker

Tillandsia hammeri Rauh et Ehlers

Tillandsia heterophylla E. Morren

Tillandsia hintoniana L.B. Sm.

Tillandsia huajuapanensis Ehlers et Lautner

(OAX)

Tillandsia huamelulaensis Ehlers

Tillandsia hubertiana Matuda (GRO)

Tillandsia ilseana W. Till, Halbritter et Zecher

(GRO)

Tillandsia imperialis E. Morren ex Mez (A) 
Apéndice 1. Continuación.

Tillandsia inopinata Espejo, López-Ferr. et W. Till

Tillandsia intermedia $\mathrm{Mez}$

Tillandsia intumescens L.B. Sm.

Tillandsia jaguactalensis I. Ramírez, Carnevali et

F. Chi (QROO)

Tillandsia jaliscomonticola Matuda

Tillandsia jaliscopinicola L. Hrom. et P. Schneid. (JAL)

Tillandsia juerg-rutschmannii Rauh (CHIS)

Tillandsia kalmbacheri Matuda

Tillandsia karwinskyana Schult. et Schult.f.

Tillandsia kirchhoffiana Wittm.

Tillandsia klausii Ehlers (CHIS)

** Tillandsia kolbii W. Till et Schatzl

Tillandsia lagunaensis Ehlers (OAX)

** Tillandsia lampropoda L.B. Sm. var. major

L.B. Sm. (A)

Tillandsia langlasseana $\mathrm{Mez}$

Tillandsia laui Matuda

** Tillandsia lautneri Ehlers

Tillandsia leucolepis L.B. Sm. (OAX)

Tillandsia limbata Schltdl.

Tillandsia loma-blancae Ehlers et Lautner (JAL)

Tillandsia loxichaensis Ehlers (OAX)

** Tillandsia lucida E. Morren ex Baker

Tillandsia lydiae Ehlers (GRO)

Tillandsia macdougallii L.B. Sm.

Tillandsia macrochlamys Baker

** Tillandsia macropetala Wawra

Tillandsia macvaughii Espejo et López-Ferr.

Tillandsia magnispica Espejo et López-Ferr.

(OAX)

** Tillandsia magnusiana Wittm.

Tillandsia makoyana Baker

Tillandsia makrinii L. Hrom. (OAX)

Tillandsia marabascoensis Ehlers et Lautner

Tillandsia maritima Matuda

** Tillandsia mateoensis Ehlers

** Tillandsia matudae L.B. Sm.

Tillandsia maya I. Ramírez et Carnevali (YUC)

Tillandsia may-patii I. Ramírez et Carnevali

(QROO)

Tillandsia mazatlanensis Rauh (SIN)

Tillandsia mirabilis L. Hrom. (GRO)

Tillandsia mitlaensis W. Weber et Ehlers (OAX)
Tillandsia mixtecorum Ehlers et Koide (OAX)

Tillandsia mooreana L.B. Sm.

Tillandsia moronesensis Ehlers (ZAC)

Tillandsia nicolasensis Ehlers (JAL)

Tillandsia nidus Rauh et J. Lehm.

Tillandsia nolleriana Ehlers

Tillandsia novakii $\mathrm{H}$. Luther (VER)

Tillandsia nuyooensis Ehlers (OAX)

Tillandsia oaxacana L.B. Sm.

Tillandsia occulta H. Luther (SIN)

** Tillandsia orogenes Standl. et L.O. Williams

Tillandsia ortgiesiana E. Morren ex Mez (A)

Tillandsia pacifica Ehlers (JAL)

Tillandsia pamelae Rauh (JAL)

Tillandsia paraisoensis Ehlers (GRO)

Tillandsia parryi Baker

Tillandsia penascoensis Ehlers et Lautner (OAX)

Tillandsia pentasticha Rauh et Wülfinghoff

(GRO)

Tillandsia pinicola I. Ramírez et Carnevali

(OAX)

Tillandsia polita L.B. Sm. var. elongata Ehlers

(A)

** Tillandsia polita L.B. Sm. var. polita (A)

** Tillandsia ponderosa L.B. Sm. (A)

Tillandsia pringlei $\mathrm{S}$. Watson

Tillandsia prodigiosa (Lem.) Baker

** Tillandsia pseudobaileyi C.S. Gardner ssp. pseudobaileyi

Tillandsia pseudobaileyi C.S. Gardner ssp.

yucatanensis I. Ramírez, Carnevali et Olmsted

Tillandsia pseudooaxacana Ehlers

Tillandsia pseudosetacea Ehlers et Rauh (OAX)

Tillandsia pueblensis L.B. Sm. (A)

Tillandsia quaquaflorifera Matuda (GRO)

Tillandsia rayonesnsis Ehlers (NL)

Tillandsia rectifolia C.A. Wiley

** Tillandsia remota Wittm.

Tillandsia rettigiana $\mathrm{Mez}$

Tillandsia rhodocephala Ehlers et Koide (OAX)

** Tillandsia rodrigueziana $\mathrm{Mez}$

Tillandsia roland-gosselinii Mez (COL)

Tillandsia roseoscapa Matuda (PUE)

Tillandsia roseospicata Matuda

Tillandsia rothii Rauh

** Tillandsia rotundata (L.B. Sm.) C.S. Gardner 
Apéndice 1. Continuación.

Tillandsia rubrispica Ehlers et Koide (OAX)
Tillandsia salmonea Ehlers (CHIS)
Tillandsia santosiae Ehlers (OAX)
Tillandsia schatzlii Rauh (OAX)
Tillandsia schusteri Rauh $(\mathbf{O A X )}$
** Tillandsia seleriana Mez
Tillandsia sessemocinoi López-Ferr., Espejo et P.
$\quad$ Blanco
Tillandsia setiformis Ehlers (OAX)

Tillandsia sierrahalensis Espejo et López-Ferr. (JAL)

Tillandsia socialis L.B. Sm. (A)

** Tillandsia streptophylla Scheidw. ex E.

Morren

Tillandsia subinflata L.B. Sm. (ZAC)

Tillandsia suesilliae W.Till, López-Ferr. et Espejo

Tillandsia superinsignis Matuda (MÉX)

Tillandsia supermexicana Matuda var. pendula

L. Hrom. (OAX)

Tillandsia supermexicana Matuda var. saxicola

L. Hrom. (GRO)

Tillandsia supermexicana Matuda var.

supermexicana

Tillandsia takizawae Ehlers et H. Luther (PUE)

Tillandsia taxcoensis Ehlers (GRO)

Tillandsia tehuacana I. Ramírez et Carnevali

Tillandsia teloloapanensis Ehlers et Lautner

(GRO)

Tillandsia thyrsigera E. Morren ex Baker (MÉX)

Tillandsia tillii Ehlers (JAL)

Tillandsia tonalaensis Ehlers (OAX)

Tillandsia trauneri L. Hrom.

Tillandsia ulrici Ehlers (OAX)

** Tillandsia velickiana L.B. Sm.

** Tillandsia velutina Ehlers

Tillandsia ventanaensis Ehlers et Koide (DGO)

** Tillandsia verapazana Ehlers

Tillandsia vernardoi Rauh (OAX)

** Tillandsia vicentina Standl.

Tillandsia violacea Baker

** Tillandsia viridiflora (Beer) Baker

Tillandsia vriesioides Matuda (CHIS)

Tillandsia weberi L. Hrom. et P. Schneid. (JAL)

Tillandsia wuelfinghoffii Ehlers (OAX)

** Tillandsia xerographica Rohweder

Tillandsia yerba-santae Ehlers (OAX)
** Tillandsia yunckeri L.B. Sm.

Tillandsia yutaninoensis Ehlers et Lautner

(OAX)

Tillandsia zacualpanensis Ehlers et Wülfingh. (MÉX)

Tillandsia zaragozaensis Ehlers (NL)

Tillandsia zoquensis Ehlers (CHIS)

Ursulaea macvaughii (L.B. Sm.) Read et Baensch

Ursulaea tuitensis (Magaña et E.J. Lott) Read et

Baensch

Viridantha atroviridipetala (Matuda) Espejo

Viridantha curvifolia (Ehlers et Rauh) López-

Ferr. et Espejo

Viridantha ignesiae (Mez) Espejo

Viridantha lepidosepala (L.B. Sm.) Espejo

Viridantha mauryana (L.B. Sm.) Espejo

Viridantha plumosa (Baker) Espejo

Viridantha tortilis (Klotzsch ex Baker) Espejo

Vriesea malzinei E. Morren (A)

** Werauhia noctiflorens Krömer, Espejo, López-

Ferr. et Acebey

Werauhia nocturna (Matuda) J.R. Grant

** Werauhia pectinata (L.B. Sm.) J.R. Grant

** Werauhia pycnantha (L.B. Sm.) J.R. Grant

Werauhia vanhyningii (L.B. Sm.) J.R. Grant

\section{Commelinaceae}

Callisia fragrans (Lindl.) Woodson

** Callisia gentlei Matuda var. elegans

(Alexander) D.R. Hunt

** Callisia gentlei Matuda var. gentlei

Callisia gentlei Matuda var. macdougallii

(Miranda) D.R. Hunt (CHIS)

Callisia hintoniorum B.L. Turner (NL)

Callisia insignis C.B. Clarke

Callisia laui (D.R. Hunt) D.R. Hunt

* Callisia micrantha (Torr.) D.R. Hunt

** Callisia multiflora (M. Martens et Galeotti)

Standl.

Callisia navicularis (Ortgies) D.R. Hunt

** Callisia soconucensis Matuda

Callisia tehuantepecana Matuda (OAX)

** Callisia warscewicziana (Kunth et Bouché)

D.R. Hunt

Commelina bambusifolia Matuda (MÉX)

Commelina bravoa Matuda 
Apéndice 1. Continuación.

Commelina congestispatha López-Ferr., Espejo
et Ceja
* Commelina dianthifolia Delile
Commelina jaliscana Matuda
Commelina nivea López-Ferrari, Espejo et Ceja (DGO)

Commelina pallida Willd.

Commelina queretarensis López-Ferr., Espejo et Ceja (QRO)

Commelina ramosissima López-Ferr., Espejo et Ceja (GTO)

Commelina rzedowskii López-Ferrari, Espejo et Ceja (MÉX)

Commelina scabra Benth.

Commelina socorrogonzaleziae Espejo et LópezFerr.

Gibasis chihuahuensis (Standl.) Rohweder

Gibasis consobrina D.R. Hunt

Gibasis gypsophila B.L. Turner (NL)

Gibasis hintoniorum B.L. Turner (NL)

Gibasis karwinskyana (Schult. et Schult.f.)

Rohweder ssp. karwinskyana

Gibasis karwinskyana (Schult. et Schult.f.)

Rohweder ssp. palmeri D.R. Hunt

Gibasis linearis (Benth.) Rohweder ssp. linearis

Gibasis linearis (Benth.) Rohweder ssp.

rhodantha (Torr.) D.R. Hunt

Gibasis lundellii (Standl.) López-Ferr. et Espejo

Gibasis matudae D.R. Hunt

Gibasis oaxacana D.R. Hunt (OAX)

** Gibasis pellucida (M. Martens et Galeotti)

D.R. Hunt

Gibasis pulchella (Kunth) Raf.

** Gibasis triflora (M. Martens et Galeotti) D. R. Hunt

Gibasis venustula (Kunth) D.R. Hunt ssp. peninsulae D.R. Hunt (BCS)

Gibasis venustula (Kunth) D.R. Hunt ssp. robusta D.R. Hunt

Gibasis venustula (Kunth) D.R. Hunt ssp. venustula

Gibasoides laxiflora (C.B. Clarke) D.R. Hunt

Matudanthus nanus (M. Martens et Galeotti) D.R. Hunt (OAX)

Thyrsanthemum floribundum (M. Martens et Galeotti) Pichon
Thyrsanthemum goldianum D.R. Hunt

Thyrsanthemum macrophyllum (Greenm.)

Rohweder

Tinantia macrophylla S. Watson

Tinantia pringlei (S. Watson) Rohweder

Tradescantia andrieuxii C.B. Clarke

Tradescantia brachyphylla Greenm.

* Tradescantia brevifolia (Torr.) Rose

* Tradescantia buckleyi (I.M. Johnst.) D.R. Hunt

Tradescantia burchii D.R. Hunt (JAL)

Tradescantia crassifolia Cav. ssp. acaulis (M.

Martens et Galeotti) C.B. Clarke

*** Tradescantia crassifolia Cav. ssp. crassifolia

Tradescantia exaltata D.R. Hunt

Tradescantia gentryi D.R. Hunt (SIN)

** Tradescantia guatemalensis C.B. Clarke

Tradescantia guiengolensis Matuda (OAX)

Tradescantia gypsophila B.L. Turner

Tradescantia hirta D.R. Hunt

** Tradescantia huehueteca (Standl. et Steyerm.)

D.R. Hunt

* Tradescantia leiandra Torr.

Tradescantia llamasii Matuda (MÉX)

Tradescantia macvaughii D.R. Hunt

Tradescantia masonii Matuda (SIN)

Tradescantia maysillesii Matuda (DGO)

Tradescantia mirandae Matuda (GRO)

Tradescantia monosperma Brandegee

Tradescantia nuevoleonensis Matuda

Tradescantia orchidophylla Rose et Hemsl.

Tradescantia peninsularis Brandegee (BCS)

* Tradescantia pinetorum Greene

Tradescantia plusiantha Standl.

Tradescantia pygmaea D.R. Hunt (DGO)

Tradescantia rozynskii Matuda

Tradescantia sillamontana Matuda (NL)

Tradescantia stenophylla Brandegee

Tradescantia tepoxtlana Matuda

* Tradescantia wrightii Rose et Bush

** Tradescantia zebrina hort. ex Bosse var.

flocculosa (G. Brückn.) D.R. Hunt

Tradescantia zebrina hort. ex Bosse var. mollipila D.R. Hunt (CHIS)

Tripogandra amplexans Handlos

** Tripogandra amplexicaulis (Klotzsch ex C.B.

Clarke) Woodson 
Apéndice 1. Continuación.

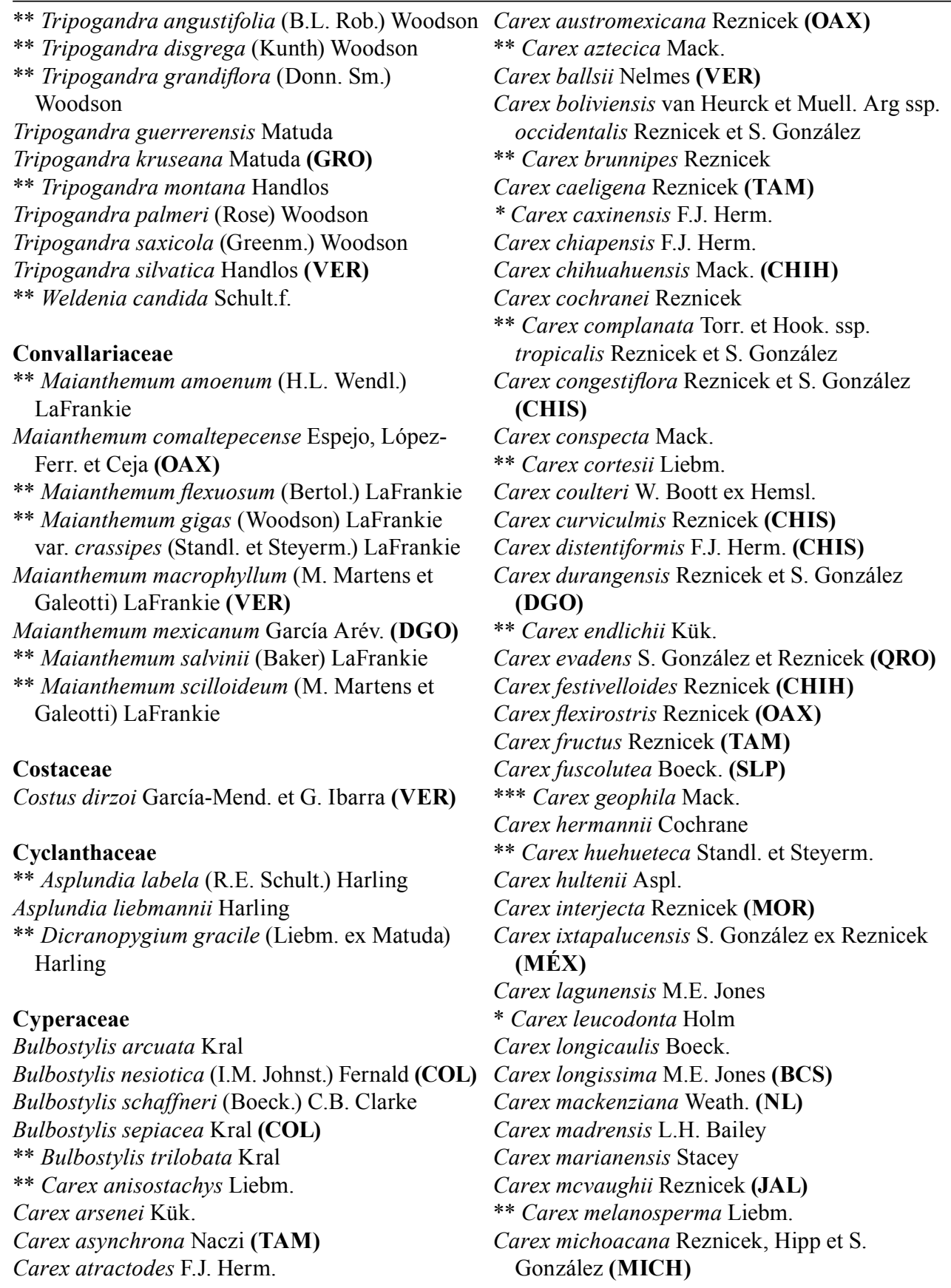


Apéndice 1. Continuación.

\author{
Carex novogaliciana Reznicek (JAL) \\ ** Carex orizabae Liebm. \\ Carex percostata F.J. Herm. \\ Carex perlonga Fernald \\ ** Carex pertenuis L.H. Bailey \\ ** Carex peucophila Holm \\ Carex pinophila Reznicek et S. González (QRO) \\ * Carex planostachys Kunze \\ Carex potosina Hemsl. \\ Carex pringlei L.H. Bailey \\ ** Carex psilocarpa Steud. \\ Carex pubigluma Reznicek (CHIS) \\ Carex queretarensis Reznicek et S. González \\ (QRO)
}

Carex rhynchoperigynium S.D. Jones et Reznicek (HGO)

Carex rzedowskii Reznicek et S. González (MICH)

Carex schaffneri Boeck.

Carex schiedeana Kunze

Carex spissa L.H. Bailey var. seatoniana (L.H. Bailey) Kük.

** Carex steyermarkii Standl.

Carex tenejapensis Reznicek et S. González

\section{(CHIS)}

Carex tolucensis (F.J. Herm.) Reznicek (MÉX)

** Carex townsendii Mack.

Carex tuberculata Liebm.

** Carex tunimanensis Standl. et Steyerm.

Carex turbinata Liebm.

Carex vallicola Dewey var. hidalgensis F.J. Herm.

* Carex wootonii Mack.

** Carex xalapensis Kunth

Cyperus arsenei O'Neill et Ben. Ayers

Cyperus breedlovei G.C. Tucker (CHIS)

Cyperus calderoniae S. González

Cyperus ciliatus Jungh.

Cyperus dioicus I.M. Johnst. (BCS)

* Cyperus dipsaceus Liebm.

Cyperus duripes I.M. Johnst. (COL)

* Cyperus fendlerianus Boeck.

* Cyperus hypopitys G.C. Tucker

** Cyperus lentiginosus Millsp. et Chase

** Cyperus lundellii O'Neill

Cyperus matudae G.C. Tucker (CHIS)

** Cyperus megalanthus (Kük.) G.C. Tucker
Cyperus michoacanensis Britton ex C.B. Clarke

Cyperus nayaritensis G.C. Tucker

Cyperus penicillatus Conz. (OAX)

Cyperus pennellii O’Neill et Ben. Ayers var. australis S. González et Rzed.

Cyperus pennellii O’Neill et Ben. Ayers var. pennellii (DGO)

* Cyperus perennis (M.E. Jones) O’Neill

Cyperus pycnostachyus (Kunth) Kunth

Cyperus sanguineo-ater Boeck.

Cyperus schaffneri Boeck.

** Cyperus semiochraceus Boeck.

* Cyperus sphaerolepis Boeck.

** Cyperus svensonii G.C. Tucker

Cyperus tempeae G.C. Tucker

Cyperus wilburii G.C. Tucker (OAX)

Cypringlea analecta (Beetle) M.T. Strong

Cypringlea coahuilensis (Svenson) M.T. Strong

(COAH)

Cypringlea evadens (C.D. Adams) Reznicek et S. González

Eleocharis arsenifera S. González, J.A. Tena et T. Alarcón (CHIH)

* Eleocharis brachycarpa Svenson

* Eleocharis cancellata S. Watson

Eleocharis cryptica Saarela, P.M. Petersen, S. González et D.J. Rosen (DGO)

Eleocharis densa Benth.

Eleocharis gonzaleziae D.J. Rosen (DGO)

Eleocharis ignota S. González et Reznicek (JAL)

Eleocharis moorei M. Strong et S. González

Eleocharis reznicekii S. González, D.J. Rosen, R. Carter et P.M. Petersen (DGO)

Eleocharis rzedowskii S. González (NL)

Eleocharis subcancellata C.B. Clarke (JAL)

Eleocharis svensoniana S. González

Eleocharis tenarum S. González et M. González

** Eleocharis urceolata (Liebm.) Svenson

Eleocharis yecorensis Roalson

Fimbristylis argillicola $\mathrm{Kral}$

Fimbristylis mexicana Palla

Fimbristylis pentastachya Boeck.

Fuirena repens Boeck.

Fuirena stephani Ramos et Diego (CAM)

Karinia mexicana (C.B. Clarke ex Britton)

Reznicek et McVaugh 
Apéndice 1. Continuación.

Pycreus aschenbornianus (Boeck.) C.B. Clarke Rhynchospora angosturensis W.W. Thomas (SLP)

Rhynchospora aristata Boeck. var. suberecta Kük.

Rhynchospora durangensis Kral et W.W. Thomas (DGO)

Rhynchospora jaliscensis McVaugh (JAL)

Rhynchospora jubata Liebm.

Rhynchospora oaxacana Kral et W.W. Thomas Rhynchospora rosae W.W. Thomas (CHIS)

Rhynchospora zacualtipanensis M. Strong (HGO)

Scleria bourgeaui Boeck. Scleria hirta Boeck. (VER)

** Scleria scabriuscula Schltdl.

** Scleria schiedeana Schltdl.

\section{Dioscoreaceae}

** Dioscorea bartlettii C.V. Morton

Dioscorea berenicea McVaugh (JAL)

** Dioscorea carpomaculata O. Téllez et B.G. Schub. var. carpomaculata

Dioscorea carpomaculata O. Téllez et B.G. Schub. var. cinerea (Uline) O. Téllez et B.G. Schub.

** Dioscorea chiapasensis Matuda

Dioscorea convolvulacea Schltdl. et Cham. ssp. grandifolia (Schltdl.) Uline ex R. Knuth

Dioscorea conzattii R. Knuth (OAX)

Dioscorea cruzensis R. Knuth (VER)

Dioscorea cyphocarpa B.L. Rob. ex R. Knuth

** Dioscorea dodecasemina Caddick et Wilkin

Dioscorea dugesii B.L. Rob.

** Dioscorea escuintlensis Matuda

Dioscorea fasciculocongesta (Sosa et B.G.

Schub.) O. Téllez

** Dioscorea floribunda M. Martens et Galeotti Dioscorea galeottiana Kunth

Dioscorea gallegosii Matuda

** Dioscorea gomez-pompae O. Téllez

Dioscorea guerrerensis R. Knuth

Dioscorea hintonii R. Knuth (MÉX)

Dioscorea igualamontana Matuda (GRO)

Dioscorea insignis C.V. Morton et B.G. Schub.

Dioscorea jaliscana S. Watson
Dioscorea juxtlahuacensis (O. Téllez et Dávila)

Caddick et Wilkin (OAX)

Dioscorea longirhiza Caddick et Wilkin (GRO)

Dioscorea longituba Uline

Dioscorea matudae O. Téllez et B.G. Schub.

(QRO)

Dioscorea mcvaughii B.G. Schub. (NAY)

Dioscorea mesoamericana O. Téllez et Mart.Rodr.

Dioscorea militaris B.L. Rob.

Dioscorea minima B.L. Rob. et Seaton

Dioscorea mitis C.V. Morton

Dioscorea morelosana (Uline) Matuda

Dioscorea multinervis Benth.

** Dioscorea nelsonii Uline ex R. Knuth

Dioscorea nematodes Uline ex R. Knuth

Dioscorea oaxacensis Uline (OAX)

Dioscorea omiltemensis O. Téllez et B.G. Schub.

(GRO)

Dioscorea oreodoxa B.G. Schub. (COL)

Dioscorea orizabensis Uline ex R. Knuth

Dioscorea pallens Schltdl.

Dioscorea palmeri R. Knuth (COL)

Dioscorea pantojensis R. Knuth (MÉX)

Dioscorea platycolpota Uline ex B.L. Rob.

Dioscorea plumifera B.L. Rob.

Dioscorea pringlei B.L. Rob.

Dioscorea pumicicola Uline (MOR)

Dioscorea remotiflora Kunth

Dioscorea sanchez-colinii Matuda

Dioscorea sessiliflora McVaugh (NAY)

Dioscorea spiculoides Matuda (VER)

Dioscorea subtomentosa Miranda

Dioscorea sumiderensis B.G. Schub. et O. Téllez (CHIS)

** Dioscorea tacanensis Lundell

Dioscorea tancitarensis Matuda (MICH)

Dioscorea temascaltepecensis R. Knuth (MÉX)

Dioscorea tubiperianthia Matuda (MÉX)

Dioscorea ulinei Greenm. ex R. Knuth

Dioscorea urceolata Uline

Dioscorea uruapanensis Matuda (MICH)

Eriocaulaceae

Eriocaulon benthamii Kunth

** Eriocaulon bilobatum Morong 
Apéndice 1. Continuación.

Eriocaulon capitulatum Moldenke

** Eriocaulon ehrenbergianum Klotzsch ex Körn.

Eriocaulon guadalajarense Ruhland (JAL)

Eriocaulon jaliscanum S. Watson

Eriocaulon mexicanum Moldenke (JAL)

Eriocaulon palmeri Ruhland (DGO)

Eriocaulon pringlei S. Watson (CHIH)

Eriocaulon schiedeanum Körn.

Eriocaulon tepicanum Moldenke (NAY)

Paepalanthus chiapensis Moldenke (CHIS)

Paepalanthus mellii Moldenke (VER)

Syngonanthus davidsei Huft (CHIS)

\section{Heliconiaceae \\ ** Heliconia adflexa (Griggs) Standl. \\ ** Heliconia bourgeana Petersen \\ ** Heliconia collinsiana Griggs \\ ** Heliconia librata Griggs \\ Heliconia mooreana R.R. Sm. \\ ** Heliconia schiedeana Klotzsch \\ Heliconia uxpanapensis C. Gut.-Báez}

\section{Hyacinthaceae}

Hemiphylacus alatostylus L. Hern.

Hemiphylacus hintoniorum L. Hern. (NL)

Hemiphylacus latifolius S. Watson (COAH)

Hemiphylacus mahindae L. Hern.

Hemiphylacus novogalicianus L. Hern. (AGS)

\section{Hypoxidaceae}

Hypoxis colliculata Sánckez-Ken

Hypoxis lucens McVaugh

Hypoxis mexicana Schult. et Schult.f.

Hypoxis potosina Brackett

Hypoxis pulchella G.L. Nesom

Hypoxis tepicensis Brackett

\section{Iridaceae}

Ainea conzattii (R.C. Foster) Ravenna (OAX) (A) * Alophia drummondii (Graham) R.C. Foster Alophia intermedia (Ravenna) Goldblatt (SIN) Alophia veracruzana Goldblatt et T.M. Howard (VER)

Calydorea longispatha (Herb.) Baker var. longispatha
Calydorea mexicana (R.C. Foster) Goldblatt et Henrich (MÉX)

Colima convoluta (Ravenna) Aarón Rodr. et L. Ortiz-Cat.

Colima tuitensis Aarón Rodr. et L. Ortiz-Cat. (JAL)

Cypella mexicana C.V. Morton et R.C. Foster

Cypella rosei R.C. Foster

Fosteria oaxacana Molseed (OAX) (A)

Nemastylis tenuis (Herb.) Baker var. caerulescens (Greenm.) R.C. Foster (MOR)

Nemastylis tenuis (Herb.) Baker var. nana (S. Watson) R.C. Foster

Nemastylis tenuis (Herb.) Baker var. pringlei (S. Watson) R.C. Foster

Nemastylis tenuis (Herb.) Baker var. purpusii R.C. Foster (SLP)

** Nemastylis tenuis (Herb.) Baker var. tenuis

Orthrosanthus exsertus (R.C. Foster) Ravenna

Rigidella flammea Lindl. (MICH) (Pr)

** Rigidella immaculata Herb.

Rigidella inusitata Cruden (GRO) (Pr)

** Rigidella orthantha Lem. (Pr)

Sessilanthera citrina Cruden (GRO)

Sessilanthera heliantha (Ravenna) Cruden (Pr)

** Sessilanthera latifolia (Weath.) Molseed et Cruden

Sisyrinchium abietum McVaugh (JAL)

Sisyrinchium angustissimum (B.L. Rob. et Greenm.) Greenm. et C.H. Thomps.

Sisyrinchium arguellesiae Ceja, Espejo et LópezFerr.

* Sisyrinchium arizonicum Rothr.

*** Sisyrinchium biforme E.P. Bicknell

Sisyrinchium bracteatum Greenm. (OAX)

Sisyrinchium cernuum (E.P. Bicknell) Kearney

Sisyrinchium cholewae Espejo, López-Ferr. et Ceja (DGO)

Sisyrinchium conzattii Calderón et Rzed.

Sisyrinchium coulterianum Klatt (HGO)

* Sisyrinchium demissum Greene

** Sisyrinchium exalatum B.L. Rob. et Greenm.

Sisyrinchium guanajuatense Ceja, Espejo et López-Ferr. (GTO)

Sisyrinchium hintoniorum G.L. Nesom (NL)

Sisyrinchium longipes (E.P. Bicknell) Kearney et Peebles 
Apéndice 1. Continuación.

Sisyrinchium longispathum Conz.

Sisyrinchium macrophyllum Greenm.

Sisyrinchium microbracteatum G.L. Nesom (NL)

Sisyrinchium novoleonense G.L. Nesom et L.

Hern.

Sisyrinchium palmeri Greenm.

Sisyrinchium parvum (E.P. Bicknell) Espejo et López-Ferr. (COAH)

Sisyrinchium planicola Ceja et Cholewa (OAX)

Sisyrinchium platyphyllum S. Watson (JAL)

Sisyrinchium polycladon B.L. Rob. et Greenm.

Sisyrinchium pringlei B.L. Rob. et Greenm.

Sisyrinchium quadrangulatum Klatt

Sisyrinchium scabrum Schltdl. et Cham.

Sisyrinchium schaffneri S. Watson

Sisyrinchium serrulatum (E.P. Bicknell) Espejo et López Ferr.

** Sisyrinchium subcernuum (E.P. Bicknell)

Henrich et Goldblatt

Sisyrinchium tolucense Peyr.

Sisyrinchium translucens (E.P. Bicknell) Espejo et López Ferr. (BCS)

Sisyrinchium zamudioi Espejo, López-Ferr. et Ceja (SLP)

Tigridia alpestris Molseed

Tigridia amatlanensis Aarón Rodr. et García-

Mend. (OAX)

Tigridia augusta Drapiez

Tigridia bicolor Molseed (OAX) (Pr)

Tigridia catarinensis Cruden (SLP)

Tigridia chiapensis Molseed ex Cruden (CHIS)

Tigridia chrysantha Cruden et S.J. Walker (JAL)

Tigridia dugesii S. Watson

Tigridia durangensis Molseed ex Cruden (DGO)

Tigridia ehrenbergii (Schltdl.) Molseed

Tigridia estelae López-Ferr. et Espejo (DGO)

Tigridia galanthoides Molseed

Tigridia gracielae Aarón Rodr. et L. Ortiz-Cat. (MÉX)

** Tigridia hallbergii Molseed

Tigridia hintonii Molseed (GRO) (Pr)

Tigridia huajuapanensis Molseed ex Cruden (Pr)

Tigridia illecebrosa Cruden

Tigridia mariaetrinitatis Espejo et López-Ferr.

(OAX)
Tigridia martinezii Calderón (HGO)

Tigridia matudae Molseed (MÉX)

** Tigridia meleagris (Lindl.) G. Nicholson

Tigridia mexicana Molseed

** Tigridia molseediana Ravenna

Tigridia mortonii Molseed (MÉX)

Tigridia multiflora (Herb.) Ravenna

** Tigridia pavonia (L.f.) DC.

Tigridia potosina López-Ferr. et Espejo (SLP)

Tigridia pugana Aarón Rodr. et L. Ortiz-Cat.

(JAL)

Tigridia pulchella B.L. Rob.

Tigridia purpusii Molseed (PUE)

Tigridia rzedowskiana Aarón Rodr. et L. Ortiz-

Cat. (QRO)

** Tigridia seleriana (Loes.) Ravenna

Tigridia suarezii Aarón Rodr. et L. Ortiz-Cat.

(JAL)

Tigridia tepoxtlana Ravenna (MOR)

Tigridia vanhouttei (Baker) Espejo et López Ferr. ssp. roldanii Molseed (HGO)

Tigridia vanhouttei (Baker) Espejo et López Ferr. ssp. vanhouttei

Tigridia venusta Cruden (MICH)

Trimezia sobolifera Ravenna

\section{Juncaceae}

** Juncus aemulans Liebm.

Juncus albicans Fernald (CHIH)

Juncus chiapasensis Balslev (CHIS)

** Luzula caricina E. Mey.

\section{Liliaceae}

Calochortus balsensis García-Mend.

Calochortus barbatus (Kunth) J.H. Painter ssp. barbatus

Calochortus barbatus (Kunth) J.H. Painter ssp. chihuahuanus J.H. Painter

Calochortus cernuus J.H. Painter

Calochortus exilis J.H. Painter (HGO)

Calochortus foliosus Ownbey (MICH) (Pr)

Calochortus fuscus Schult.f.

** Calochortus ghiesbreghtii S. Watson

Calochortus hartwegii Benth.

Calochortus hintonii Bullock ex Ownbey (MÉX)

Calochortus marcellae G.L. Nesom 
Apéndice 1. Continuación.

\author{
Calochortus mendozae Espejo, López-Ferr. et \\ Ceja (SLP) \\ Calochortus nigrescens Ownbey (Pr) \\ Calochortus pringlei B.L. Rob. \\ Calochortus purpureus (Kunth) Baker \\ Calochortus venustulus Greene
}

\section{Marantaceae}

Calathea atropurpurea Matuda

Calathea leonoriae Lascur., H. Oliva et Avendaño (VER)

** Calathea matudae H. Kenn. et Ganders

Calathea misantlensis Lascur. (VER)

Calathea ovandensis Matuda

** Calathea soconuscum Matuda

Calathea sousandreana H. Kenn. et Ganders

Stromanthe popolucana Cast.-Campos, Vovides et Vázq. Torres (VER)

\section{Melanthiaceae}

Anticlea frigida (Schltdl. et Cham.) Zomlefer et Judd

Anticlea hintoniorum (B.L. Turner) Zomlefer et Judd

Anticlea neglecta (Espejo, López-Ferr. et Ceja)

Zomlefer et Judd

* Anticlea virescens (Kunth) Rydb. (Pr)

** Anticlea volcanica (Benth.) Baker

Schoenocaulon calcicola Greenm. (OAX)

Schoenocaulon caricifolium (Schltdl.) A. Gray

Schoenocaulon comatum Brinker

Schoenocaulon conzattii Brinker (OAX)

Schoenocaulon framei Zomlefer et Judd

Schoenocaulon ghiesbreghtii Greenm.

Schoenocaulon ignigenum Frame

Schoenocaulon intermedium Baker

Schoenocaulon jaliscense Greenm. var. jaliscense (Pr)

Schoenocaulon jaliscense Greenm. var. regulare (Brinker) Frame (Pr)

Schoenocaulon macrocarpum Brinker

Schoenocaulon madidorum Frame

Schoenocaulon megarrhizum M.E. Jones

Schoenocaulon mortonii Brinker

Schoenocaulon oaxacense (Frame) Zomlefer et Judd (OAX)
Schoenocaulon obtusum Brinker (HGO)

Schoenocaulon pellucidum Frame

Schoenocaulon plumosum Frame

Schoenocaulon pringlei Greenm. (Pr)

Schoenocaulon rzedowskii Frame

Schoenocaulon tenorioi Frame

Schoenocaulon tenue Brinker

Schoenocaulon tenuifolium (M. Martens et

Galeotti) B.L. Rob. et Greenm.

* Schoenocaulon texanum Scheele

Schoenocaulon tigrense Frame (JAL)

\section{Nolinaceae}

Beaucarnea compacta L. Hern. et Zamudio (GTO)

** Beaucarnea goldmanii Rose (A)

Beaucarnea gracilis Lem. (A)

Beaucarnea hiriartiae L. Hern. (GRO) (A)

** Beaucarnea pliabilis (Baker) Rose (A)

Beaucarnea recurvata Lem. (A)

Beaucarnea sanctomariana L. Hern. (OAX) (A)

Beaucarnea stricta Lem. (A)

Calibanus glassianus L. Hern. et Zamudio

(GTO)

Calibanus hookeri (Lem.) Trel. (A)

Dasylirion acrotriche (Schiede) Zucc. (A)

Dasylirion berlandieri S. Watson (NL) (Pr)

Dasylirion cedrosanum Trel.

Dasylirion gentry Bogler

Dasylirion glaucophyllum Hook. (HGO)

Dasylirion graminifolium (Zucc.) Karw. et Zucc.

* Dasylirion leiophyllum Engelm. ex Trel.

Dasylirion longissimum Lem. (A)

Dasylirion longistylum J.F. MacBr. (SLP)

Dasylirion lucidum Rose

Dasylirion miquihuanense Bogler

Dasylirion occidentalis Bogler ex Hochstätter

\section{(AGS)}

Dasylirion palaciosii Rzed. (SLP)

Dasylirion parryanum Trel. (SLP)

Dasylirion quadrangulatum S. Watson (A)

Dasylirion sereke Bogler (CHIH)

Dasylirion serratifolium (Karw.) Zucc. (OAX)

Dasylirion simplex Trel. (DGO)

* Dasylirion texanum Scheele

* Dasylirion wheeleri S. Watson 
Apéndice 1. Continuación.

Nolina azureogladiata D. Donati (OAX)

Nolina beldingii Brandegee (BCS)

* Nolina bigelovii (Torr.) S. Watson

Nolina cespitifera Trel.

Nolina durangensis Trel.

Nolina elegans Rose

* Nolina erumpens (Torr.) S. Watson

Nolina hibernica Hochstätter et D. Donati (TAM)

Nolina humilis S. Watson

Nolina juncea (Zucc.) J.F. MacBr.

Nolina longifolia (Karw.) Hemsl.

Nolina matapensis Wiggins (SON)

Nolina micrantha I.M. Johnst.

* Nolina microcarpa S. Watson

Nolina nelsonii Rose (TAM)

Nolina palmeri S. Watson (BC)

Nolina parviflora (Kunth) Hemsl.

Nolina pumila Rose

Nolina rigida (Brongn.) Trel.

* Nolina texana $\mathrm{S}$. Watson

\section{Orchidaceae}

** Acianthera angustifolia (Lindl.) Luer

** Acianthera angustisepala (Ames et Correll) Pridgeon et M.W. Chase

** Acianthera breedlovei Soto Arenas, Solano et Salazar

Acianthera chrysantha (Lindl.) Pridgeon et M.W. Chase

Acianthera eximia (L.O. Williams) Solano (OAX)

(A)

Acianthera greenwoodii Soto Arenas (OAX)

Acianthera hartwegiifolia (H. Wendl. et Kraenzl.)

Solano et Soto Arenas

Acianthera majakoluckae Soto Arenas et Solano

Acianthera martinezii (Luer) Luer (CHIS)

Acianthera obscura (A. Rich. et Galeotti)

Pridgeon et M.W. Chase

Acianthera sotoana Solano

** Acianthera tikalensis (Correll et C. Schweinf.) Pridgeon et M.W. Chase

Acianthera unguicallosa (Ames et C. Schweinf.)

Solano (COL) (Pr)

** Acianthera violacea (A. Rich. et Galeotti)

Pridgeon et M.W. Chase (Pr)

** Acineta barkeri (Bateman) Lindl. (A)
Acineta hagsateri Salazar et Soto Arenas (GRO)

** Acineta salazarii Soto Arenas

Alamania punicea Lex. ssp. greenwoodiana Soto

Arenas et R. Jiménez

Alamania punicea Lex. ssp. punicea

** Anathallis comayaguensis (Ames) Pridgeon et M.W. Chase

Anathallis greenwoodii Soto Arenas et Salazar

(OAX)

Anathallis involuta (L.O. Williams) Solano et Soto Arenas

** Anathallis lewisae (Ames) Solano et Soto Arenas

** Anathallis minutalis (Lindl.) Pridgeon et M.W. Chase

Anathallis oblanceolata (L.O. Williams) Solano et Soto Arenas (A)

Anathallis scariosa (Lex.) Pridgeon et M.W.

Chase

Arpophyllum laxiflorum Pfitzer

** Arpophyllum medium Rchb.f.

Arpophyllum spicatum Lex.

Artorima erubescens (Lindl.) Dressler et G.E.

Pollard

** Aulosepalum hemichrea (Lind1.) Garay

** Aulosepalum nelsonii (Greenm.) Garay

Aulosepalum oestlundii (Burns-Bal.) Catling

(GRO)

** Aulosepalum pyramidale (Lind1.) M.A. Dix et M.W. Dix

Aulosepalum ramentaceum (Lindl.) Garay

Aulosepalum riodelayense (Burns-Bal.) Salazar

(OAX)

Aulosepalum tenuiflorum (Greenm.) Garay

Barkeria barkeriola Rchb.f.

Barkeria dorotheae Halb. (A)

Barkeria fritz-halbingeriana Soto Arenas (OAX)

Barkeria melanocaulon A. Rich. et Galeotti

(OAX) (A)

Barkeria naevosa (Lindl.) Schltr.

Barkeria palmeri (Rolfe) Schltr.

Barkeria scandens (Lex.) Dressler et Halb. (Pr)

Barkeria shoemakeri Halb. (Pr)

** Barkeria skinneri (Bateman ex Lindl.) A.

Rich. et Galeotti (Pr)

** Barkeria spectabilis Bateman ex Lindl. 
Apéndice 1. Continuación.

\begin{tabular}{|c|c|}
\hline Barkeria strophinx (Rchb.f.) Halb. (MICH) (A) & ** Camaridium pulchrum Schltr. \\
\hline Barkeria uniflora (Lex.) Dressler et Halb. & Camaridium rhombeum (Lindl.) M.A. Blanco \\
\hline Barkeria vanneriana Rchb.f. & Camaridium soconuscanum (Breedlove et Mally) \\
\hline Barkeria whartoniana (C. Schweinf.) Soto & M.A. Blanco (CHIS) \\
\hline Arenas $(\mathbf{O A X})(\mathbf{P r})$ & ** Campylocentrum microphyllum Ames et \\
\hline ** Beloglottis mexicana Garay et Hamer & Correll \\
\hline Bletia adenocarpa Rchb.f. & ** Catasetum integerrimum Hook. \\
\hline Bletia amabilis C. Schweinf. & Catasetum laminatum Lindl. \\
\hline Bletia coccinea Lex. & Catasetum pendulum Dodson \\
\hline Bletia concolor Dressler & ** Chondrorhyncha lendyana Rchb.f. \\
\hline Bletia ensifolia L.O. Williams & Chysis addita Dressler (CHIS) \\
\hline ** Bletia gracilis G. Lodd. & ** Chysis bractescens Lindl. (A) \\
\hline Bletia greenmaniana L.O. Williams & ** Chysis laevis Lindl. \\
\hline Bletia greenwoodiana Sosa (DGO) & Chysis limminghei Linden et Rchb.f. (TAB) (A) \\
\hline ** Bletia jucunda Linden et Rchb.f. & Clowesia dodsoniana E. Aguirre \\
\hline Bletia lilacina A. Rich. et Galeotti & Clowesia glaucoglossa (Rchb.f.) Dodson \\
\hline Bletia macristhmochila Greenm. & (MICH) (Pr) \\
\hline Bletia neglecta Sosa & Clowesia rosea Lindl. (A) \\
\hline Bletia nelsonii Ames & Clowesia thylaciochila (Lem.) Dodson \\
\hline Bletia parkinsonii Hook. & ** Coelia densiflora Rolfe (Pr) \\
\hline Bletia punctata Lex. & ** Coelia guatemalensis Rchb.f. \\
\hline ** Bletia reflexa Lindl. & ** Corallorhiza bulbosa A. Rich. et Galeotti \\
\hline Bletia riparia Sosa et Palestina (VER) & Corallorhiza ehrenbergii Rchb.f. \\
\hline ** Bletia roezlii Rchb.f. & Corallorhiza fimbriata Schltr. \\
\hline Bletia similis Dressler (MICH) & Corallorhiza macrantha Schltr. (Pr) \\
\hline ** Bletia tenuifolia Ames et C. Schweinf. & Corallorhiza pringlei Greenm. \\
\hline Bletia urbana Dressler (A) & Corallorhiza williamsii Correll (MOR) \\
\hline Bletia villae Soto Arenas (DGO) & ** Cranichis apiculata Lindl. \\
\hline Bletia warfordiana Sosa (JAL) & Cranichis ciliilabia C. Schweinf. \\
\hline $\begin{array}{l}\text { Brachystele luzmariana Szlach. et R. González } \\
\text { (JAL) }\end{array}$ & $\begin{array}{l}\text { ** Cranichis cochleata Dressler } \\
\text { Cranichis gracilis L.O. Williams }\end{array}$ \\
\hline $\begin{array}{l}\text { Brachystele tamayoana Szlach., Rutk. et Mytnik } \\
\text { (BC) }\end{array}$ & $\begin{array}{l}\text { ** Cranichis hieroglyphica Ames et Correll } \\
\text { ** Cranichis revoluta Hamer et Garay }\end{array}$ \\
\hline ** Brassia brachiata Lindl. & Cranichis subumbellata A. Rich. et Galeotti \\
\hline Brassia signata Rchb.f. & ** Cranichis sylvatica A. Rich. et Galeotti \\
\hline Bulbophyllum cirrhosum L.O. Williams & ** Cuitlauzina candida (Lindl.) Dressler et N.H. \\
\hline Bulbophyllum nagelii L.O. Williams & Williams (A) \\
\hline Bulbophyllum solteroi $\mathrm{R}$. González & Cuitlauzina dubia (S. Rosillo) E. Yáñez et Soto \\
\hline Camaridium atratum (Lex.) M.A. Blanco & Arenas ex Solano \\
\hline ** Camaridium densum (Lindl.) M.A. Blanco & Cuitlauzina pendula Lex. (A) \\
\hline $\begin{array}{l}\text { ** Camaridium hagsaterianum (Soto Arenas) } \\
\text { M.A. Blanco }\end{array}$ & $\begin{array}{l}\text { ** Cuitlauzina pulchella (Bateman ex Lindl.) } \\
\text { Dressler et N.H. Williams }\end{array}$ \\
\hline ** Camaridium meleagris (Lindl.) M.A. Blanco & Cuitlauzina pygmaea (Lindl.) M.W. Chase et N.H. \\
\hline Camaridium oestlundianum (L.O. Williams) & Williams \\
\hline M.A. Blanco (GRO) & ** Cyclopogon comosus (Rchb.f.) Burns-Bal. et \\
\hline ** Camaridium praestans (Rchb.f.) M.A. Blanco & E.W. Greenw. \\
\hline
\end{tabular}


Apéndice 1. Continuación.

Cyclopogon luteo-albus (A. Rich. et Galeotti)

Schltr.

** Cyclopogon obliquus (J.J. Sm.) Szlach.

** Cyclopogon papilio Szlach.

Cyclopogon pringlei (S. Watson) Soto Arenas

Cyclopogon saccatus A. Rich. et Galeotti

** Cycnoches ventricosum Bateman (A)

** Cypripedium dickinsonianum Hágsater (Pr)

** Cypripedium irapeanum Lex. (A)

Cypripedium molle Lindl.

Deiregyne albovaginata (C. Schweinf.) Garay

Deiregyne chartacea (L.O. Williams) Garay (JAL)

Deiregyne densiflora (C. Schweinf.) Salazar et Soto Arenas

Deiregyne diaphana (Lindl.) Garay (OAX)

** Deiregyne eriophora (B.L. Rob. et Greenm.) Garay

Deiregyne falcata (L.O. Williams) Garay

Deiregyne nonantzin (R. González ex McVaugh)

Catling (JAL)

Deiregyne pandurata Garay (DGO)

Deiregyne pseudopyramidalis (L.O. Williams) Garay

Deiregyne ramirezii R. González (JAL)

Deiregyne rhombilabia Garay

Deiregyne sheviakiana (Szlach.) Espejo et López

Ferr. (CHIS)

Deiregyne tenorioi Soto Areanas et Salazar

Deiregyne velata (B.L. Rob. et Fernald) Garay

(CHIH)

** Dichaea intermedia Ames et Correll

** Dichromanthus aurantiacus (Lex.) Salazar et

Soto Arenas

*** Dichromanthus cinnabarinus (Lex.) Garay

** Dichromanthus michuacanus (Lex.) Salazar et Soto Arenas

Dichromanthus yucundaa Salazar et García-

Mend. (OAX)

Domingoa gemma (Rchb.f.) van der Berg et Soto Arenas (PUE)

Domingoa kienastii (Rchb.f.) Dressler

** Domingoa purpurea Lindl.

** Dryadella greenwoodiana Soto Arenas,

Salazar et Solano

** Dryadella linearifolia (Ames) Luer
Elleanthus teotepecensis Soto Arenas (GRO)

Encyclia adenocarpa (Lex.) Schltr.

Encyclia adenocaula (Lex.) Schltr. ssp. adenocaula (A)

Encyclia adenocaula (Lex.) Schltr. ssp. kennedyi (Fowlie et Withner) Soto Arenas (A) Encyclia aenicta Dressler et G.E. Pollard

** Encyclia ambigua (Lindl.) Schltr.

** Encyclia asperula Dressler et G.E. Pollard Encyclia atrorubens (Rolfe) Schltr. (Pr)

** Encyclia belizensis (Rchb.f.) Schltr.

** Encyclia bractescens (Lindl.) Hoehne

Encyclia calderoniae Soto Arenas (OAX)

Encyclia candollei (Lindl.) Schltr.

Encyclia chiapasensis Withner et D.G. Hunt (CHIS)

Encyclia contrerasii R. González (COL)

** Encyclia diota (Lind1.) Schltr.

Encyclia flabellata (Lindl.) B. Thurst. et W.

Thurst. (VER)

** Encyclia guatemalensis (Klotzsch) Dressler et G.E. Pollard

Encyclia halbingeriana Hágsater et Soto Arenas (MICH)

** Encyclia hanburyi (Lindl.) Schltr.

Encyclia huertae Soto Arenas et R. Jiménez (MICH)

** Encyclia incumbens (Lindl.) Mabb.

Encyclia kienastii (Rchb.f.) Dressler et G.E. Pollard (OAX) (P)

Encyclia lorata Dressler et G.E. Pollard (GRO) (Pr)

Encyclia meliosma (Rchb.f.) Schltr.

Encyclia microbulbon (Hook.) Schltr.

Encyclia nizandensis Pérez-García et Hágsater (OAX)

Encyclia oestlundii (Ames, F.T. Hubb. et C.

Schweinf.) Hágsater et Stermitz (GRO)

Encyclia ovulum (Lindl.) Schltr. (SIN)

** Encyclia papillosa (Bateman) Aguirre-Olav.

Encyclia parviflora (Regel) Whitner

Encyclia pollardiana (Withner) Dressler et G.E. Pollard (Pr)

Encyclia rzedowskiana Soto Arenas (OAX)

Encyclia spatella (Rchb.f.) Schltr. (JAL)

Encyclia suaveolens Dressler 
Apéndice 1. Continuación.

Encyclia trachycarpa (Lindl.) Schltr.

Encyclia uxpanapensis Salazar (VER)

Epidendrum alabastrialatum G.E. Pollard ex

Hágsater (Pr)

** Epidendrum alticola Ames et Correll (A)

** Epidendrum alvarezdeltoroi Hágsater

Epidendrum anisatum Lex.

** Epidendrum arbusculum Lindl.

** Epidendrum atroscriptum Hágsater

** Epidendrum beharorum Hágsater

Epidendrum camposii Hágsater (OAX)

** Epidendrum cerinum Schltr. (Pr)

Epidendrum chimalapense Hágsater et Salazar

** Epidendrum chloe Rchb.f. (Pr)

Epidendrum chlorops Rchb.f.

Epidendrum cilioccidentale Hágsater et L.

Sánchez

Epidendrum citrosmum Hágsater

** Epidendrum clowesii Bateman ex Lindl.

** Epidendrum cnemidophorum Lindl. (A)

Epidendrum costatum A. Rich. et Galeotti (OAX)

** Epidendrum culmiforme Schltr.

Epidendrum cusii Hágsater

** Epidendrum cystosum Ames (Pr)

** Epidendrum dixiorum Hágsater

Epidendrum dorsocarinatum Hágsater (MÉX) (Pr)

Epidendrum dressleri Hágsater (Pr)

Epidendrum erectifolium Hágaster et L. Sánchez

(CHIS)

** Epidendrum eustirum Ames, F.T. Hubb. et C.

Schweinf.

Epidendrum examinis S. Rosillo (JAL)

** Epidendrum eximium L.O. Williams

Epidendrum falcatum Lindl.

** Epidendrum fruticosum Pav. ex Lindl.

** Epidendrum galeottianum A.Rich. et Galeotti

Epidendrum gasteriferum Scheeren (OAX)

Epidendrum gomezii Schltr.

Epidendrum gonzalez-tamayoi Hágsater

Epidendrum greenwoodii Hágsater (OAX)

Epidendrum guerrerense Hágsater et GarcíaCruz (GRO)

Epidendrum hagsateri Christenson

Epidendrum hueycatenangense Hágsater et

García-Cruz (GRO)
Epidendrum ibarrae R. González (JAL)

Epidendrum incomptoides Ames, F.T. Hubb. et C. Schweinf. (CHIS) (Pr)

Epidendrum juergensenii Rchb.f. (OAX)

** Epidendrum lacertinum Lindl.

Epidendrum lignosum Lex.

Epidendrum longicaule (L.O. Williams) L.O.

Williams

Epidendrum longipetalum A. Rich. et Galeotti

Epidendrum lowilliamsii García-Cruz

Epidendrum macdougallii (Hágsater) Hágsater

Epidendrum macroclinium Hágsater

Epidendrum magnificum Schltr. (GRO)

Epidendrum marmoratum A. Rich. et Galeotti

** Epidendrum martinezii L. Sánchez et

Carnevali

Epidendrum matudae L.O. Williams

Epidendrum melistagoides Hágstaer et L.

Sánchez

** Epidendrum melistagum Hágsater

** Epidendrum mesocarpum Hágsater

** Epidendrum microcharis Rchb.f.

Epidendrum miserum Lindl.

Epidendrum mixtecanum Hágsater et García-

Cruz (OAX)

** Epidendrum mixtum Schltr.

Epidendrum mocinoi Hágsater

Epidendrum motozintlensis Hágstaer et L.

Sánchez (CHIS)

** Epidendrum myrianthum Lindl.

Epidendrum nagelii L.O. Williams (GRO)

** Epidendrum nelsonii Hágsater

Epidendrum neogaliciense Hágsater et R.

González (JAL)

** Epidendrum nitens Rchb.f.

Epidendrum oaxacanum Rolfe (OAX)

Epidendrum pastranae Hágsater

Epidendrum pollardii Hágsater

** Epidendrum polychromum Hágsater

** Epidendrum propinquum A. Rich. et Galeotti

** Epidendrum radioferens (Ames, F.T. Hubb. et

C. Schweinf.) Hágsater

** Epidendrum roseoscriptum Hágsater

Epidendrum rosilloi Hágsater

Epidendrum rowleyi Withner et G.E. Pollard

** Epidendrum singuliflorum Schltr. 
Apéndice 1. Continuación.

** Epidendrum skutchii Ames, F.T. Hubb. et C. Schweinf. (Pr)

** Epidendrum sobralioides Ames et Correll

(A)

Epidendrum stallforthianum Kraenzl. (VER)

Epidendrum succulentum Hágsater

** Epidendrum tacanaense Hágsater, Soto

Arenas et E. Santiago A.

Epidendrum tortipetalum Scheeren

Epidendrum tuxtlense Hágsater, García-Cruz et

L. Sánchez

Epidendrum tziscaoense Hágsater (CHIS)

Epidendrum vandifolium Lindl.

** Epidendrum veroscriptum Hágsater

** Epidendrum verrucipes Schltr.

** Epidendrum wendtii Hágsater et Salazar

Erycina echinata (Kunth) Lindl.

Erycina hyalinobulbon (Lex.) N.H. Williams et M.W. Chase

** Eurystyles borealis A.H. Heller (Pr)

** Funkiella hyemalis (A. Rich. et Galeotti) Schltr.

Funkiella laxispica (Catling) Salazar et Soto Arenas (OAX)

Funkiella markowskiana (Szlach.) Salazar et Soto Arenas (OAX)

Funkiella porphyricola (Schltr.) Salazar et Soto Arenas

Funkiella rubrocallosa (B.L. Rob. et Greenm.)

Salazar et Soto Arenas

Galeandra greenwoodiana Warford

Galeoglossum cactorum Salazar et ChávezRendón (OAX)

Galeoglossum prescottioides A. Rich. et Galeotti (OAX)

Galeoglossum thysanochilum (B.L. Rob. et Greenm.) Salazar (OAX)

Galeoglossum tubulosum (Lindl.) Salazar et Soto Arenas

Galeottia grandiflora A. Rich. (P)

Galeottiella sarcoglossa (A. Rich. et Galeotti)

Schltr. (Pr)

** Gongora galeata (Lindl.) Rchb.f.

Gongora galeottiana A. Rich.

** Gongora tridentata Whitten (Pr)

** Gongora truncata Lindl.
Goodyera brachyceras (A. Rich. et Galeotti)

Garay et G.A. Romero (OAX)

Goodyera dolabripetala (Ames) Schltr.

Goodyera fimbrilabia Ormerod (CHIS)

Goodyera purpusii Ormerod (VER)

Goodyera zacuapanensis Ormerod (VER)

Govenia alba A. Rich. et Galeotti

Govenia bella E.W. Greenw. (OAX)

Govenia capitata Lindl.

** Govenia dresslerana E.W. Greenw.

Govenia elliptica S. Watson (NL)

** Govenia greenwoodii Dressler et Soto Arenas (GRO)

Govenia jouyana R. González (JAL)

** Govenia lagenophora Lindl.

** Govenia liliacea (Lex.) Lindl.

** Govenia matudae E.W. Greenw. et Soto

Arenas

** Govenia mutica Rchb.f.

Govenia praecox Salazar et E.W. Greenw. (VER)

Govenia purpusii Schltr.

Govenia rubellilabia García-Cruz (CHIS)

Govenia tequilana Dressler et Hágsater (Pr)

** Guarianthe aurantiaca (Bateman ex Lindl.)

Dressler et W.E. Higgins

** Guarianthe bowringiana (H.J. Veitch ex

O'Brien) Dressler et W.E. Higgins

Habenaria acalcarata Espejo et López Ferr.

(CHIS)

Habenaria agapitae R. González et Reynoso

(JAL)

Habenaria agrestis R. González et Cuev.-Fig.

(JAL)

Habenaria atrata R. González et Cuev.-Fig. (JAL)

Habenaria brevilabiata A. Rich. et Galeotti

** Habenaria brownelliana Catling

Habenaria calicis R. González (MOR)

Habenaria carvajaliana R. González et Cuev.-

Fig. (JAL)

Habenaria castroi R. González et Cuev.-Fig.

(ZAC)

Habenaria cortesii R. González et Cuev.-Fig.

(JAL)

** Habenaria crassicornis Lindl.

Habenaria cuevasiana R. González et Cuev.-Fig.

(JAL) 
Apéndice 1. Continuación.

\begin{tabular}{l}
\hline Habenaria diffusa A. Rich et Galeotti (MÉX) \\
Habenaria entomantha (Lex.) Lindl. \\
Habenaria felipensis Ames (OAX) \\
Habenaria filifera S. Watson \\
Habenaria flexuosa Lindl. (OAX) \\
Habenaria gonzaleztamayoi García-Cruz, R. \\
$\quad$ Jiménez et L. Sánchez \\
Habenaria greenwoodiana R. González (JAL) \\
** Habenaria guadalajarana S. Watson \\
Habenaria horaliae R. González (MICH) \\
Habenaria ibarrae R. González (JAL) \\
Habenaria ixtlanensis E.W. Greenw. (OAX) \\
** Habenaria jaliscana S. Watson \\
Habenaria jardeliana R. González et Cuev.-Fig. \\
(JAL)
\end{tabular}

Habenaria kariniae R. González et Cuev.-Fig.

Habenaria leon-ibarrae R. Jiménez et Carnevali

(QROO)

Habenaria lizbethae R. González et Cuev.-Fig.

(JAL)

Habenaria luzmariana R. González (JAL)

Habenaria mariae R. González et Cuev.-Fig. (JAL)

Habenaria matudae Salazar

Habenaria mcvaughiana R. González

Habenaria micheliana R. González et Cuev.-Fig. (JAL)

Habenaria mitodes Garay et W. Kittr.

Habenaria nogeirana R. González et Cuev.-Fig.

(JAL)

** Habenaria novemfida Lindl.

** Habenaria odontopetala Rchb.f.

Habenaria oreophila Greenm.

Habenaria ortiziana R. González (MÉX)

Habenaria pinzonii R. González et Cuev.-Fig.

Habenaria pseudofilifera R. González et Cuev.-

Fig. (JAL)

Habenaria rosilloana R. González (MICH)

Habenaria rosulifolia Espejo et López-Ferr.

(MOR)

Habenaria rotundifolia Conz. (OAX)

Habenaria ruizii R. González (JAL)

Habenaria rzedowskiana R. González

Habenaria rzedowskii R. González (MICH)

Habenaria schaffneri $\mathrm{S}$. Watson

Habenaria socorroae R. González et Cuev.-Fig.

(JAL)
** Habenaria spithamaea Schltr.

** Habenaria strictissima Rchb.f.

Habenaria subauriculata B.L. Rob. et Greenm.

Habenaria szlachetkoana R. González et Cuev.-

Fig. (JAL)

Habenaria talaensis R. González et Cuev.-Fig.

(JAL)

Habenaria tequilana R. González et Cuev.-Fig. (JAL)

** Habenaria tetranema Schltr.

Habenaria uncata R. Jiménez, L. Sánchez et García-Cruz (MOR)

Habenaria virens A. Rich. et Galeotti

Habenaria xochitliae R. González (JAL)

Habenaria zamudioana R. González (MICH)

Hagsatera brachycolumna (L.O. Williams) R. González (Pr)

Hagsatera rosilloi R. González (JAL)

Helleriella guerrerensis Dressler et Hágsater

(GRO)

Hexalectris brevicaulis L.O. Williams

Hexalectris fallax M.I. Rodríguez et R. González

* Hexalectris grandiflora (A. Rich. et Galeotti)

L.O. Williams

* Hexalectris nitida L.O. Williams

Hexalectris parviflora L.O. Williams

Hexalectris revoluta Correll

* Hexalectris spicata (Walter) Barnhart var. arizonica (S. Watson) Catling et V.S. Engel

* Hexalectris warnockii Ames et Correll

Hintonella mexicana Ames

Homalopetalum pachyphyllum (L.O. Williams)

Dressler

Homalopetalum pumilum (Ames) Dressler

** Isochilus alatus Schltr.

** Isochilus aurantiacus Hamer et Garay

Isochilus bracteatus (Lex.) López-Ferr. et Espejo

Isochilus langlassei Schltr.

Isochilus oaxacanus Salazar et Soto Arenas

(OAX)

Isochilus unilateralis B.L. Rob.

Jacquiniella cernua (Lindl.) Dressler

** Jacquiniella cobanensis (Ames et Schltr.) Dressler

** Jacquiniella gigantea Dressler, Salazar et

García-Cruz (Pr) 
Apéndice 1. Continuación.

** Kefersteinia tinschertiana Pupulin (Pr)

Kionophyon pollardiana Szlach., Rutk., et Mytnik (OAX)

Kionophyton sawyeri (Standl. et L.O. Williams) Garay

** Kionophyton seminuda (Schltr.) Garay

Kraenzlinella hintonii (L.O. Williams) Solano (GRO) (Pr)

Kreodanthus casillasii R. González (JAL)

** Kreodanthus ovatilabius (Ames et Correll) Garay

** Lacaena bicolor Lindl. (A)

Laelia albida Bateman ex Lindl.

Laelia anceps Lindl. (P)

Laelia aurea Navarro

Laelia autumnalis (Lex.) Lindl.

Laelia crawshayana Rchb. (JAL)

Laelia eyermaniana Rchb.f.

Laelia furfuracea Lindl. (OAX)

Laelia gouldiana Rchb.f. (HGO) (E)

Laelia speciosa (Kunth) Schltr. (Pr)

** Laelia superbiens Lindl. (A)

Leochilus carinatus (Knowles et Westc.) Lindl.

Leochilus crocodiliceps (Rchb.f.) Kraenzl.

** Leochilus johnstonii Ames et Correll

** Leochilus oncidioides Knowles et Westc.

** Lepanthes acuminata Schltr. ssp. acuminata

Lepanthes acuminata Schltr. ssp. ernestii Salazar et Soto Arenas (CHIS)

** Lepanthes almololongae Luer et Béhar

Lepanthes ancylopetala Dressler (CHIS) (Pr)

** Lepanthes appendiculata Ames

Lepanthes aprica Catling et V.R. Catling (OAX)

Lepanthes attenuata Salazar, Soto Arenas et O. Suárez

Lepanthes avis Rchb.f.

Lepanthes brachystele Salazar et Soto Arenas

Lepanthes breedlovei Salazar et Soto Arenas

Lepanthes calopetala Salazar et Soto Arenas

Lepanthes camposii Salazar et Soto Arenas

Lepanthes catlingii Salazar, Soto Arenas et O. Suárez (OAX)

Lepanthes chiangii Salazar, Soto Arenas et O. Suárez (OAX)

Lepanthes cryptostele Salazar et Soto Arenas (GRO)
** Lepanthes denticulata Luer et Béhar

Lepanthes erythroxantha Salazar et Soto Arenas (OAX)

Lepanthes gabriellae Salazar et Soto Arenas (OAX)

Lepanthes galeottiana Salazar et Soto Arenas (OAX)

Lepanthes greenwoodii Salazar et Soto Arenas (OAX)

** Lepanthes guatemalensis Schltr. (Pr)

Lepanthes guerrerensis Salazar et Soto Arenas

Lepanthes hagsateri Salazar et Soto Arenas

(GRO)

** Lepanthes johnsonii Ames

** Lepanthes lenticularis Luer et Béhar

Lepanthes machorroi Salazar et Soto Arenas

(OAX)

Lepanthes maldonadoae Soto Arenas (CHIS)

Lepanthes mariae Salazar et Soto Arenas (OAX)

Lepanthes martinezii Salazar et Soto Arenas

Lepanthes matudana Salazar et Soto Arenas

(CHIS)

Lepanthes maxima Salazar et Soto Arenas (CHIS)

Lepanthes mazatlanensis Solano et Reynaud (OAX)

Lepanthes minima Salazar, Soto Arenas et O. Suárez (OAX)

Lepanthes mixe Salazar et Soto Arenas (OAX)

Lepanthes moorei C. Schweinf.

Lepanthes motozintlensis Salazar et Soto Arenas

(CHIS)

Lepanthes nagelii Salazar et Soto Arenas

Lepanthes nigriscapa R.E. Schult. et G.W. Dillon (OAX)

Lepanthes oaxacana Salazar, Soto Arenas et O. Suárez (OAX)

** Lepanthes oreocharis Schltr.

Lepanthes oreophila Catling et V.R. Catling (OAX)

Lepanthes papilionacea Salazar, Soto Arenas et O. Suárez (OAX)

** Lepanthes papillipetala Dressler

** Lepanthes parvula Dressler (Pr)

Lepanthes pollardii Hespenh. (OAX)

Lepanthes rekoi R.E. Schult. 
Apéndice 1. Continuación.

** Lepanthes samacensis Ames
Lepanthes schiedei Rchb.f.
Lepanthes schultesii Salazar et Soto Arenas
(OAX)
** Lepanthes scopula Schltr.
Lepanthes sousae Salazar et Soto Arenas (OAX)
** Lepanthes stenophylla Schltr.
Lepanthes suarezii Salazar et Soto Arenas (OAX)
** Lepanthes tecpanica Luer et Béhar
** Lepanthes tenuiloba R.E. Schult. et G.W.
Dillon
Lepanthes thurstoniorum Salazar, Soto Arenas et
O. Suárez

Lepanthes totontepecensis Salazar et Soto Arenas (OAX)

Lepanthes vivipara Salazar et Soto Arenas (CHIS)

Lepanthes wendtii Salazar et Soto Arenas (OAX)

** Lepanthes williamsii Salazar et Soto Arenas

** Lepanthes yunckeri Ames

Lepanthes yuvilensis Catling

Liparis cordiformis C. Schweinf.

Liparis draculoides E.W. Greenw.

** Liparis fantastica Ames et C. Schweinf.

Liparis greenwoodiana Espejo

Liparis lindeniana (A. Rich. et Galeotti) Hemsl. (VER)

Liparis madrensis Soto Arenas, Salazar et R. Jiménez (DGO)

Liparis volcanica $\mathrm{R}$. González et Zamudio Lockhartia galeottiana A. Rich. ex Soto Arenas

** Lockhartia oerstedii Rchb.f.

** Lockhartia verrucosa Lindl. ex Rchb.f.

** Lycaste aromatica (Graham) Lindl.

** Lycaste cochleata Lindl.

** Lycaste consobrina Rchb.f.

Lycaste crinita Lindl.

** Lycaste cruenta (Lindl.) Lindl.

** Lycaste deppei (Lodd.) Lindl.

** Lycaste lassioglossa Rchb.f. (P)

** Lycaste skinneri (Bateman ex Lindl.) Lindl. (P)

** Macroclinium bicolor (Lindl.) Dodson

Macroclinium lexarzanum (Hágsater et R. González) Dodson

Macroclinium pachybulbon (Hágsater et R. González) Dodson
Malaxis abieticola Salazar et Soto Arenas

** Malaxis acianthoides Schltr.

Malaxis adenotropa R. González, Lisb. Hern. et E. Ramírez (JAL)

Malaxis alvaroi García-Cruz, R. Jiménez et L. Sánchez

Malaxis amplexicolumna E. Greenw. et R. González (CHIS)

Malaxis andersoniana R. González, Lisb. Hern. et E. Ramírez (MÉX)

Malaxis brachystachya (Rchb.f.) Kuntze

Malaxis casillasii R. González (JAL)

Malaxis chiarae R. González, Lisb. Hern. et E.

Ramírez (JAL)

Malaxis chica Todzia (NL)

Malaxis contrerasii R. González (JAL)

** Malaxis corymbosa (S. Watson) Kuntze

Malaxis elliptica A. Rich. et Galeotti (MOR)

Malaxis elviae R. González (JAL)

Malaxis espejoi R. González, Lizb. Hern. \& E. Ramírez (JAL)

** Malaxis fastigiata (Rchb.f.) Kuntze

Malaxis greenwoodiana Salazar et Soto Arenas (CHIS) (Pr)

Malaxis hagsateri Salazar (GRO) (Pr)

Malaxis hintonii Todzia

Malaxis javesiae (Rchb.f.) Ames

** Malaxis lepanthiflora (Schltr.) Ames

** Malaxis lepidota (Finet) Ames

Malaxis lizbethiae R. González, Lisb. Hern. et E. Ramírez (JAL)

Malaxis luceroana R. González

Malaxis lyonnetii Salazar (MOR)

Malaxis macrostachya (Lex.) Kuntze

Malaxis macvaughiana R. González, Lisb. Hern. et E. Ramírez (JAL)

Malaxis marthaleidae R. González, Lisb. Hern. et E. Ramírez (JAL)

Malaxis martinezii R. González (JAL)

Malaxis micheliana R. González, Lisb. Hern. et

E. Ramírez (JAL)

Malaxis molotensis Salazar et de Santiago (GRO)

Malaxis myurus (Lindl.) Kuntze

Malaxis nelsonii Ames (DGO)

Malaxis novogaliciana McVaugh

Malaxis ocreata (S. Watson) Ames 
Apéndice 1. Continuación.

Malaxis perezii R. González (JAL)

Malaxis pringlei (S. Watson) Ames

Malaxis ramirezii R. González (JAL)

Malaxis reichei (Schltr.) Ames et C. Schweinf.

Malaxis ribana Espejo et López-Ferr. (MOR)

Malaxis roblesgilana R. González (NAY)

Malaxis rodrigueziana $\mathrm{R}$. González

Malaxis rosei Ames

Malaxis rosilloi R. González et E.W. Greenw.

Malaxis rositae R. González, Lisb. Hern. et E. Ramírez (JAL)

Malaxis ruizii R. González (JAL)

Malaxis rzedowskiana R. González (MÉX)

Malaxis salazarii Catling

Malaxis streptopetala (B.L. Rob. et Greenm.)

Ames

Malaxis stricta L.O. Williams (MOR)

Malaxis tamayoana Garay et W. Kittr. (JAL)

Malaxis tepicana Ames

Malaxis tequilensis R. González, Lisb. Hern. et

E. Ramírez (JAL)

** Malaxis triangularis Dressler

Malaxis urbana E.W. Greenw. (OAX)

Malaxis wendtii Salazar

Malaxis xerophila Salazar et L.I. Cabrera

Malaxis zempoalensis López-Ferr. et Espejo (MOR)

** Masdevallia adamsii Luer

** Masdevallia floribunda Lindl.

** Masdevallia tuerckheimii Ames

** Maxillariella cobanensis (Schltr.) M.A. Blanco et Carnevali

** Maxillariella houtteana (Rchb.f.) M.A. Blanco et Carnevali

Maxillariella mexicana (J.T. Atwood) M.A.

Blanco et Carnevali

** Maxillariella tenuifolia (Lindl.) M.A. Blanco et Carnevali

Meiracyllium gemma Rchb.f.

** Mesadenella petenensis (L.O. Williams) Garay Mesadenus chiangii (M. Johnst.) Garay (COAH)

** Mesadenus polyanthus (Rchb.f.) Schltr.

Mesadenus tenuissimus (L.O. Williams) Garay

(MOR)

Mexipedium xerophyticum (Soto Arenas, Salazar et Hágsater) V.A. Albert et M.W. Chase (OAX) (P)
** Microchilus luniferus (Schltr.) Ormerod

Microchilus mexicanus (Ames) Ormerod (CHIS)

Microepidendrum subulatifolium (A. Rich. et

Galeotti) W.E. Higgins

** Mormodes aromatica Lindl.

Mormodes badia Rolfe ex W. Watson

Mormodes cozticxochitl Salazar

** Mormodes lineata Bateman ex Lindl.

Mormodes luxata Lindl.

Mormodes maculata (Klotzsch) L.O. Williams var. maculata (OAX) (A)

Mormodes maculata (Klotzsch) L.O. Williams var. unicolor (Hook.) L.O. Williams (A)

** Mormodes nagelii L.O. Williams

Mormodes oestlundiana Salazar et Hágsater (GRO)

Mormodes pardalinata S. Rosillo

Mormodes porphyrophlebia Salazar (A)

Mormodes ramirezii S. Rosillo (JAL)

Mormodes sanguineoclaustra Fowlie (GRO) (P)

** Mormodes sotoana Salazar (P)

Mormodes tezontle S. Rosillo

Mormodes tuxtlensis Salazar (VER)

Mormodes uncia Rchb.f. (OAX) (P)

Mormodes williamsii hort. ex G. Nicholson (JAL)

Mormolyca sotoana (Carnevali et Gómez-Juárez)

M.A. Blanco (CHIS)

** Myoxanthus octomerioides (Lindl.) Luer

** Myrmecophila christinae Carnevali et GómezJuárez var. christinae

Myrmecophila christinae Carnevali et GómezJuárez var. ibarrae Carnevali et J.L. Tapia

(QROO)

Myrmecophila galeottiana (A. Rich.) Rolfe

Myrmecophila grandiflora (Lindl.) Carnevali,

J.L. Tapia et I. Ramírez

Myrmecophila laguna-herrerae Carnevali, L.

Ibarra et J.L. Tapia

Nemaconia dresslerana (Soto Arenas) van den

Berg, Salazar et Soto Arenas (MOR) (Pr)

** Nemaconia glomerata (Correll) van den Berg,

Salazar et Soto Arenas

** Nemaconia graminifolia Knowles et Westc.

Nemaconia longipetala (Correll) van den Berg,

Salzar et Soto Arenas

** Nemaconia pellita (Rchb.f.) van den Berg, Salazar et Soto Arenas (Pr) 
Apéndice 1. Continuación.

\begin{tabular}{l}
\hline Notylia leucantha Salazar (OAX) \\
** Notylia orbicularis A. Rich. et Galeotti ssp. \\
orbicularis \\
Notylia orbicularis A. Rich. et Galeotti ssp. \\
warfordiae Salazar \\
Notylia tamaulipensis Rchb.f. (TAM) \\
** Ocampoa mexicana A. Rich. et Galeotti \\
Oestlundia cyanocolumna (Ames, F.T. Hubb. et \\
C. Schweinf.) W.E. Higgins \\
Oestlundia distantiflora (A. Rich. et Galeotti) \\
W.E. Higgins (Pr) \\
Oestlundia luteorosea (A. Rich. et Galeotti) W.E. \\
Higgins \\
Oestlundia tenuissima (Ames, F.T. Hubb. et C. \\
Schweinf.) W.E. Higgins \\
Oncidium brachyandrum Lindl. \\
Oncidium durangense Hágsater \\
** Oncidium endocharis Rchb.f. (A) \\
Oncidium geertianum C. Morren
\end{tabular}

Oncidium ghiesbreghtianum A. Rich. et Galeotti

Oncidium graminifolium (Lindl.) Lindl.

Oncidium hagsaterianum R. Jiménez et Soto Arenas (VER)

Oncidium hastatum (Bateman) Lindl.

Oncidium hintonii L.O. Williams

Oncidium incurvum F. Barker ex Lindl. (A)

Oncidium karwinskii (Lindl.) Lindl.

** Oncidium laeve (Lind1.) Beer

Oncidium leleui R. Jiménez et Soto Arenas

** Oncidium leucochilum Lindl. (A)

Oncidium lindleyi (Galeotti ex Lindl.) R. Jiménez et Soto Arenas

** Oncidium maculatum (Lind1.) Lindl.

Oncidium microstigma Rchb.f. (MOR)

Oncidium oblongatum Lindl.

** Oncidium oliganthum (Rchb.f.) L.O. Williams

Oncidium oviedomotae Hágsater (MICH)

** Oncidium pergamineum Lindl.

Oncidium pollardii Dodson et Hágsater (OAX) (A)

Oncidium reflexum Lindl.

Oncidium reichenheimii (Linden et Rchb.f.) Garay et Stacy

Oncidium sotoanum R. Jiménez et Hágsater ssp. papalosmum R. Jiménez

** Oncidium sphacelatum Lindl.
Oncidium stelligerum Rchb.f. (Pr)

Oncidium suave Lindl.

** Oncidium subcruciforme (A.H. Heller) Salazar

** Oncidium suttonii Lindl. (Pr)

** Oncidium tenuipes Kraenzl.

Oncidium tigrinum Lex. (A)

Oncidium unguiculatum Lindl. (A)

** Oncidium wentworthianum Lindl. (Pr)

Ornithidium tonsoniae (Soto Arenas) Senghas

(Pr)

Ornithocephalus biloborostratus Salazar et R.

González (MICH)

** Ornithocephalus iridifolius Rchb.f.

Ornithocephalus obergiae Soto Arenas (VER)

Ornithocephalus torresii Salazar et Soto Arenas

(CHIS)

Ornithocephalus tripterus Schltr.

Pachyphyllum mexicanum Dressler et Hágsater

(OAX) (Pr)

Papperitzia leiboldii (Rchb.f.) Rchb.f. (Pr)

** Pelexia ghiesbreghtii Szlach., Mytnik et Rutk.

** Pelexia gutturosa (Rchb.f.) Garay

Physogyne garayana R. González et Szlach.

(COL)

Physogyne gonzalezii (L.O. Williams) Garay

(OAX) (Pr)

Physogyne sparsiflora (C. Schweinf.) Garay

* Platanthera brevifolia (Greene) Kraenzl.

Platanthera calderoniae López Ferr. et Espejo

(MICH)

** Platanthera limosa Lindl.

Platanthera nubigena A. Rich. et Galeotti (VER)

Platanthera volcanica Lindl.

** Platystele pedicellaris (Schltr.) Garay

** Platystele repens (Ames) Garay (VER) (Pr)

** Pleurothallis correllii Luer

** Pleurothallis nelsonii Ames (Pr)

** Pleurothallis pansamalae Schltr.

** Pleurothallis saccatilabia C. Schweinf. (Pr)

** Polystachya clavata Lindl.

Polystachya mcvaughiana Soto Arenas

Ponera exilis Dressler

** Ponera juncifolia Lindl.

** Ponthieva guatemalensis Rchb.f.

Ponthieva hildae R. González et Soltero (JAL)

** Ponthieva parvula Schltr. 
Apéndice 1. Continuación.

Ponthieva rinconii Salazar (VER)

Ponthieva schaffneri (Rchb.f.) E.W. Greenw.

** Ponthieva triloba Schltr.

** Ponthieva trilobata (L.O. Williams) L.O. Williams

Potosia kusibabiana Szlach., Mytnik et Rutk.

(NL)

Potosia praetermissa Szlach., Mytnik et Rutk.

(SLP)

Potosia tamayoana Szlach., Mytnik et Rutk.

Prescottia lindeniana A. Rich. et Galeotti (CHIS)

Prescottia pachyrrhiza A. Rich. et Galeotti (OAX)

Prosthechea bicamerata (Rchb.f.) W.E. Higgins

Prosthechea brachiata (A. Rich. et Galeotti) W.E. Higgins

** Prosthechea chondylobulbon (A. Rich. et Galeotti) W.E. Higgins

Prosthechea citrina (Lex.) W.E. Higgins (Pr)

Prosthechea concolor (Lex.) W.E. Higgins

Prosthechea cretacea (Dressler et G.E. Pollard) W.E. Higgins

Prosthechea favoris (Rchb.f.) W.E. Higgins

Prosthechea fragrans (Sw.) W.E. Higgins

Prosthechea ghiesbreghtiana (A. Rich. et

Galeotti) W.E. Higgins

** Prosthechea glauca (Knowles et Westc.) W.E. Higgins

Prosthechea greenwoodiana (Aguirre-Olav.) W.E. Higgins (OAX)

Prosthechea hastata (Lindl.) W.E. Higgins

Prosthechea karwinskii (Mart.) Soto Arenas et Salazar (OAX)

Prosthechea linkiana (Klotzsch) W.E. Higgins

** Prosthechea maculosa (Ames, F.T. Hubb. et C. Schweinf.) W.E. Higgins

** Prosthechea madrensis (Schltr.) Karremans

Prosthechea magnispatha (Ames, F.T. Hubb. et C. Schweinf.) W.E. Higgins

Prosthechea mariae (Ames) W.E. Higgins (A)

** Prosthechea michuacana (Lex.) W.E. Higgins

Prosthechea mulasii Soto Arenas et L. Cerv.

(GRO)

Prosthechea obpiribulbon (Hágsater) W.E. Higgins
** Prosthechea panthera (Rchb.f.) W.E. Higgins

Prosthechea pastoris (Lex.) Espejo et López-Ferr. Prosthechea pringlei (Rolfe) W.E. Higgins

Prosthechea pterocarpa (Lindl.) W.E. Higgins

Prosthechea punctulata (Rchb.f.) Soto Arenas et

Salazar

** Prosthechea rhynchophora (A. Rich. et Galeotti) W.E. Higgins

Prosthechea semiaperta (Hágsater) W.E. Higgins

Prosthechea tripunctata (Lindl.) W.E. Higgins

Prosthechea trulla (Rchb.f.) W.E. Higgins

Prosthechea varicosa (Bateman ex Lindl.) W.E.

Higgins ssp. leiobulbon (Hook.) Dressler et

G.E. Pollard

** Prosthechea vitellina (Lindl.) Dressler (Pr)

Pseudogoodyera pseudogoodyerioides (L.O.

Williams) R. González et Szlach. (Pr)

** Rhyncholaelia digbyana (Lindl.) Schltr.

** Rhyncholaelia glauca (Lindl.) Schltr.

Rhynchostele aptera (Lex.) Soto Arenas et Salazar

Rhynchostele candidula (Rchb.f.) Soto Arenas et Salazar

Rhynchostele cervantesii (Lex.) Soto Arenas et Salazar (A)

Rhynchostele cordata (Lindl.) Soto Arenas et Salazar (A)

Rhynchostele ehrenbergii (Link, Klotzsch et Otto) Soto Arenas et Salazar (A)

Rhynchostele galeottiana (A. Rich. et Galeotti)

Soto Arenas et Salazar (Pr)

Rhynchostele londesboroughiana (Rchb.f.) Soto Arenas et Salazar (GRO) (A)

Rhynchostele maculata (Lex.) Soto Arenas et Salazar

Rhynchostele madrensis (Rchb.f.) Soto Arenas et Salazar (A)

** Rhynchostele majalis (Rchb.f.) Soto Arenas et Salazar (P)

** Rhynchostele pygmaea (Lindl.) Rchb.f. (Pr)

** Rhynchostele rossii (Lindl.) (A)

** Rhynchostele stellata (Lindl.) Soto Arenas et Salazar

** Rhynchostele uroskinneri (Lindl.) Soto Arenas et Salazar (P)

Rodriguezia dressleriana R. González (Pr) 
Apéndice 1. Continuación.

\section{** Rossioglossum grande (Lindl.) Garay et H.A. Kenn. (P) \\ Rossioglossum hagsaterianum Soto Arenas (NAY)}

Rossioglossum insleayi (Barker ex Lindl.) Garay et H.A. Kenn. (A)

Rossioglossum splendens (Rchb.f.) Garay et H.A. Kenn. (A)

** Rossioglossum williamsianum (Rchb.f.) Garay et H.A. Kenn. (P)

** Sarcoglottis assurgens (Rchb.f.) Schltr.

** Sarcoglottis cerina (Lindl.) P.N. Don (Pr)

** Sarcoglottis corymbosa Garay

Sarcoglottis lobata (Lind1.) P.N. Don (HGO)

** Sarcoglottis richardiana (Schltr.) Soto Arenas

** Sarcoglottis rosulata (Lindl.) P.N. Don

** Sarcoglottis schaffneri (Rchb.f.) Ames

Sarcoglottis scintillans (E.W. Greenw.) Salazar et Soto Arenas

** Scaphyglottis confusa (Schltr.) Ames et Correll

** Scaphyglottis hondurensis (Ames) L.O. Williams

Schiedeella affinis (C. Schweinf.)

Schiedeella crenulata (L.O. Williams) Espejo et López-Ferr.

Schiedeella durangensis (Ames et C. Schwienf.) Buns.-Bal.

Schiedeella garayana R. González (JAL)

Schiedeella llaveana (Lindl.) Schltr. var. alinae Szlach.

Schiedeella llaveana (Lindl.) Schltr. var. guerrerensis Szlach. (GRO)

Schiedeella nagelii (L.O. Williams) Garay (SLP) (Pr)

Schiedeella romeroana Szlach. (OAX)

Schiedeella tenella (L.O. Williams) Burns.-Bal.

** Schiedeella trilineata (Lindl.) Burns.-Bal.

Schiedeella williamsiana Szlach., Rutk. et Mytnik (JAL)

Sigmatostalix mexicana L.O. Williams (GRO) (Pr)

Sobralia galeottiana A. Rich.

** Sobralia macdougallii Soto Arenas, PérezGarcía et Salazar

** Sobralia macrantha Lindl.
** Sobralia xantholeuca B.S. Williams

Sotoa confusa (Garay) Salazar

** Specklinia alata (A. Rich. et Galeotti) Solano et Soto Arenas

Specklinia digitale (Luer) Pridgeon et M.W.

Chase (A)

** Specklinia fuegii (Rchb.f.) Solano et Soto Arenas

** Specklinia marginata (Lindl.) Pridgeon et M.W. Chase

** Specklinia pisinna (Luer) Solano et Soto Arenas

* Spiranthes delitescens Sheviak

Spiranthes graminea Lindl.

** Spiranthes nebulorum Catling et V.R. Catling

Stanhopea dodsoniana Salazar et Soto Arenas

Stanhopea hernandezii (Kunth) Schltr.

Stanhopea intermedia Klinge

Stanhopea maculosa Knowles et Westc.

Stanhopea martiana Lindl. ex Bateman

** Stanhopea oculata (Lodd.) Lindl. (A)

Stanhopea pseudoradiosa Jenny et R. González (OAX)

** Stanhopea radiosa Lem.

** Stanhopea ruckeri Lindl.

** Stanhopea saccata Bateman

Stanhopea tigrina Lindl. (A)

** Stanhopea whittenii Soto Arenas, Salazar et

G. Gerlach

Stelis aeolica Solano et Soto Arenas (CHIS)

Stelis annedamoniae Solano

Stelis aristocratica (L.O. Williams) Solano et Soto Arenas (GRO)

Stelis chiapensis Solano

Stelis desantiagoi Solano et Salazar (GRO)

** Stelis emarginata (Lindl.) Soto Arenas

Stelis fulva Schltr.

** Stelis greenwoodii Soto Arenas et Solano

Stelis hagsateri Solano (CHIS)

** Stelis jalapensis (Kraenzl.) Pridgeon et M.W. Chase

** Stelis johnsonii Ames

Stelis martinezii Solano

Stelis nagelii Solano

** Stelis nicaraguensis (Liebm.) Solano et Soto Arenas 
Apéndice 1. Continuación.

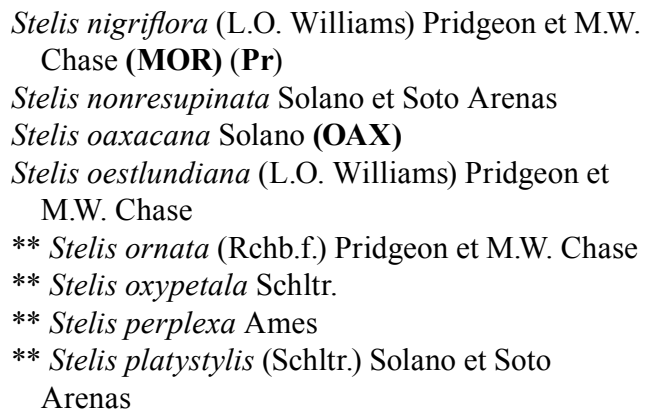

Stelis resupinata (Ames) Pridgeon et M.W. Chase Stelis retusa (Lex.) Pridgeon et M.W. Chase

Stelis rufobrunnea (Lindl.) L.O. Williams

Stelis salazarii Solano (OAX)

Stelis sanguinolenta (Garay et W. Kittr.) Solano (JAL)

Stelis soconuscana Solano (CHIS)

Stelis sotoana Solano (OAX)

Stelis sotoarenasii Solano (OAX)

** Stelis tacanensis Solano et Soto Arenas

** Stelis tenuissima Schltr.

Stelis veracrucensis Solano (OAX)

** Stelis vespertina Solano et Soto Arenas

Stelis wendtii Solano (OAX)

Stelis xerophila (Schltr.) Soto Arenas

Svenkoeltzia congestiflora (L.O. Williams) Burns.-Bal.

Svenkoeltzia luzmariana R. González (JAL)

Svenkoeltzia pamelae Szlach., Rutk. et Mytnik (OAX)

Svenkoeltzia patriciae R. González (JAL)

** Tamayorkis ehrenbergii (Rchb.f.) R. González et Szlach.

Tamayorkis hintonii (Todzia) R. González et Szlach.

* Tamayorkis porphyrea (Ridl.) Salazar et Soto Arenas

* Tamayorkis wendtii (Salazar) R. González et Szlach.

Trichocentrum andreanum (Cogn.) R. Jiménez et Carnevali

Trichocentrum andrewsiae (R. Jiménez et Carnevali) R. Jiménez et Carnevali

** Trichocentrum bicallosum (Lindl.) M.W.

Chase et N.H. Williams
Trichocentrum biorbiculare (Balam et Cetzal) R. Jiménez et Solano

** Trichocentrum candidum Lindl.

Trichocentrum cosymbephorum (Morren) R. Jiménez et Carnevali

Trichocentrum flavovirens (L.O. Williams) M.W. Chase et N.H. Williams (Pr)

Trichocentrum hoegei Rchb.f. (Pr)

** Trichocentrum lindenii (Brongn.) M.W. Chase et N.H. Williams

Trichocentrum leptotifolium (Cetzal et Carnevali) R. Jiménez et Solano

Trichocentrum longifolium (Lindl.) R. Jiménez

Trichocentrum margalefii (Hágsater) M.W. Chase et N.H. Williams

** Trichocentrum microchilum (Lindl.) M.W. Chase et N.H. Williams

Trichocentrum nataliae Balam et Carnevali (JAL)

** Trichocentrum oerstedii (Rchb.f.) R. Jiménez et Carnevali

Trichocentrum oestlundianum (L.O. Williams) M.W. Chase et N.H. Williams

** Trichocentrum pachyphyllum (Hook.) R. Jiménez et Carnevali

Trichocentrum pendulum (Carnevali et Cetzal) R. Jiménez et Solano

Trichocentrum sierracaracolense (Cetzal et Balam) R. Jiménez et Solano (CHIS)

Trichocentrum stramineum (Lindl.) M.W. Chase et N.H. Williams (VER)

Trichocentrum teboana (R. Jiménez, Carnevali et J.L. Tapia) R. Jiménez et Carnevali (TAB)

Trichocentrum yucatanense (Cetzal et Carnevali) R. Jiménez et Solano

Trichopilia galeottiana A. Rich. (P)

Trichosalpinx nageliana Soto Arenas

** Trichosalpinx pringlei (Schltr.) Luer

Trichosalpinx tamayoana Soto Arenas (COL)

Triphora mexicana (S. Watson) Schltr.

Triphora yucatanensis Ames

** Vanilla cribbiana Soto Arenas

** Vanilla insignis Ames

\section{Poaceae}

Aegopogon solisii G.A. Levin (COL) 
Apéndice 1. Continuación.

* Aegopogon tenellus (DC.) Trin. var. abortivus (E. Fourn.) Beetle Agrostis bourgaei E. Fourn. Agrostis calderoniae Acosta (MÉX) ** Agrostis ghiesbreghtii E. Fourn. ** Agrostis laxissima Swallen Agrostis liebmannii (E. Fourn.) Hitchc. Agrostis novogaliciana McVaugh (JAL) (Pr) Agrostis rosei Scribn. et Merr. Agrostis schaffneri E. Fourn. Agrostis thyrsigera $\mathrm{Mez}$

* Allolepis texana (Vasey) Soderstr. et H.F. Decker ** Andropogon bourgaei Hack.

Andropogon maderensis Swallen (COAH) Andropogon pringlei Scribn. et Merr.

* Aristida californica Thurb.

Aristida eludens Allred et Valdés-Reyna

* Aristida glabrata (Vasey) Hitchc.

Aristida gypsophila Beetle (COAH)

* Aristida havardii Vasey

Aristida hintonii Hitchc.

Aristida hitchcockiana Henrard

Aristida jaliscana R. Guzmán et V. Jaram.

** Aristida liebmannii E. Fourn.

Aristida mexicana Scribn.

* Aristida pansa Wooton et Standl.

Aristida petersonii Allred et Valdés-Reyna

(OAX)

Aristida purpusiana Hitchc. (BCS)

Aristida scribneriana Hitchc.

Aristida spanospicula Allred, Valdés-Reyna et

Sánckez-Ken

Aristida tenuifolia Hitchc. (COL)

Aristida tuitensis Sánchez-Ken et Dávila (JAL)

Aristida vaginata Hitchc. (JAL)

* Aristida wrightii Nash

Arundinella palmeri Vasey ex Beal

Aulonemia laxa (F. Maek.) McClure

** Axonopus arsenei Swallen

Axonopus deludens Chase (JAL)

Axonopus mexicanus G.A. Black

Axonopus reederi G.A. Black (CHIS)

Axonopus rosei (Scribn. et Merr.) Chase (NAY)

* Blepharidachne bigelovii (S. Watson) Hack.

Blepharoneuron shepherdii (Vasey) P.M.

Peterson et Annable
Bothriochloa hirtifolia (J. Presl) Henrard

* Bothriochloa wrightii Hack.

Bouteloua annua Swallen

Bouteloua distans Swallen

* Bouteloua eludens Griffiths

Bouteloua johnstonii Swallen (COAH)

Bouteloua karwinskii (E. Fourn.) Griffiths

** Bouteloua longiseta Gould

Bouteloua pedicellata Swallen

Bouteloua purpurea Gould et Kapadia

* Bouteloua radicosa (E. Fourn.) Griffiths

* Bouteloua ramosa Scribn. ex Vasey

Bouteloua reflexa Swallen

Bouteloua tamaulipensis G.J. Pierce ex D.L. Pacheco et Columbus (TAM)

** Bouteloua triaena (Trin.) Scribn.

* Bouteloua warnockii Gould et Kapadia

** Bouteloua williamsii Swallen

* Brachiaria arizonica (Scribn. et Merr.) S.T. Blake

Brachiaria jaliscana F. Santana (JAL)

Brachiaria meziana Hitchc.

Brachiaria ophryodes Chase

Brachypodium pringlei Scribn. ex Beal

Bromus attenuatus Swallen

Bromus densus Swallen

** Bromus dolichocarpus Wagnon

Bromus meyeri Swallen (NL)

* Bromus mucroglumis Wagnon

Bromus thysanoglottis Soderstr. et Beaman

Buchlomimus nervatus (Swallen) Reeder, C.

Reeder et Rzed.

Calamagrostis coahuilensis P.M. Peterson, Soreng et Valdés-Reyna

Calamagrostis divaricata P.M. Peterson et Soreng (DGO)

Calamagrostis erectifolia Hitchc.

Calamagrostis eriantha (Kunth) Steud.

** Calamagrostis guatemalensis Hitchc.

Calamagrostis pringlei Beal

* Calamagrostis tolucensis (Kunth) Trin. ex Steud.

Calamagrostis valida Sohns

* Calamagrostis vulcanica Swallen

** Cathestecum brevifolium Swallen

* Cathestecum erectum Vasey et Hack. 
Apéndice 1. Continuación.

Cathestecum prostratum J. Presl

Cathestecum varium Swallen

** Cenchrus multiflorus J. Pres1

Cenchrus palmeri Vasey

Chasmanthium curvifolium (Valdés-Reyna,

Morden et Hatch) Wipff et S.D. Jones (TAM)

* Chloris andropogonoides E. Fourn. (NL)

* Chloris cucullata Bisch.

* Chloris subdolichostachya Müll. Berol.

** Chloris submutica Kunth

* Chondrosum brevisetum (Vasey) Clayton

Chondrosum chasei (Swallen) Clayton (CHIH)

Chondrosum elatum (Reeder et C. Reeder) Clayton

Chondrosum eriostachyum (Swallen) Clayton (COAH)

* Chondrosum parryi E. Fourn.

Chondrosum scorpioides (Lag.) Kunth

* Chondrosum trifidum (Thurb.) Clayton

Chusquea aperta L.G. Clark (OAX)

Chusquea bilimekii E. Fourn.

Chusquea circinata Soderstr. et C.E. Calderón

Chusquea galeottiana Rupr. ex Munro

Chusquea glauca L.G. Clark

** Chusquea lanceolata Hitchc.

Chusquea mulleri Munro

** Chusquea nelsonii Scribn. et J.G. Sm.

Chusquea perotensis L.G. Clark, G. Cortés et Cházaro (VER)

Chusquea repens L.G. Clark et Londoño ssp. oaxacensis L.G. Clark et Londoño (OAX)

Chusquea repens L.G. Clark et Londoño ssp. repens (CHIS)

** Coelorachis ramosa (E. Fourn.) Nash

Ctenium planifolium (J. Presl) Kunth

Ctenium plumosum (Hitchc.) Swallen

Cyclostachya stolonifera (Scribn.) Reeder et C.

Reeder

Danthonia chiapasensis Davidse (CHIS)

Deschampsia liebmanniana (E. Fourn.) Hitchc.

Digitaria badia (Scribn. et Merr.) Fernald (CHIS)

Digitaria breedlovei R.W. Pohl et Davidse

(CHIS)

Digitaria distans (Chase) Fernald (JAL)

* Digitaria hitchcockii (Chase) Stuck.

** Digitaria leucites (Trin.) Henrard
Digitaria paniculata Soderstr. ex McVaugh

(JAL) (Pr)

* Digitaria patens (Swallen) Henrard

Distichlis bajaensis H.L. Bell (BC)

Distichlis palmeri (Vasey) Fassett

Echinochloa holciformis (Kunth) Chase

Echinochloa jaliscana McVaugh

Echinochloa oplismenoides (E. Fourn.) Hitchc.

** Echinolaena standleyi (Hitchc.) Stieber

* Elionurus barbiculmis Hack.

* Elymus arizonicus (Scribn. et J.G. Sm.) Gould

Enteropogon brandegeei (Vasey ex Brandegee)

Clayton (BCS)

*** Enteropogon chlorideus (J. Presl) Clayton

** Eragrostis contrerasii R.W. Pohl

* Eragrostis erosa Scribn. ex Beal

** Eragrostis guatemalensis Withers.

** Eragrostis hirta E. Fourn.

** Eragrostis hondurensis R.W. Pohl

* Eragrostis obtusiflora (E. Fourn.) Scribn.

* Eragrostis palmeri $\mathrm{S}$. Watson

Eragrostis plumbea Scribn. ex Beal

Eragrostis pringlei Mattei

* Eragrostis silveana Swallen

** Eriochloa lemmonii Vasey et Scribn.

Eriochloa nelsonii Scribn. et J.G. Sm. var. nelsonni

Eriochloa nelsonii Scribn. et J.G. Sm. var. papillosa R.B. Shaw

* Erioneuron avenaceum (Kunth) Tateoka var. nealleyi (Vasey) Gould

Festuca bajacaliforniana Gonz.-Led. et S.D. Koch (BC)

Festuca bidenticulata E.B. Alexeev (VER)

Festuca callosa (Piper) St.-Yves (PUE)

Festuca coahuilana Gonz.-Led. et S.D. Koch (COAH)

Festuca diclina Darbysh. (CHIH)

Festuca X gonzalez-ledesmae Darbysh.

** Festuca hephaestophila Nees ex Steud.

Festuca hintoniana E.B. Alexeev (NL)

Festuca jaliscana E.B. Alexeev

* Festuca ligulata Swallen

Festuca livida (Kunth) Willd. ex Spreng.

Festuca longiligula Darbysh. (COAH)

** Festuca lugens (E. Fourn.) Hitchc. ex Hern.Xol. 
Apéndice 1. Continuación.

Festuca X miscella Darbysh. (MÉX)

Festuca orizabensis E.B. Alexeev

Festuca pringlei St.-Yves

Festuca roblensis Gonz.-Led.

* Festuca rosei Piper

Festuca rzedowskiana E.B. Alexeev (MÉX)

Festuca tancitaroensis Gonz.-Led. et S.D. Koch (MICH)

Festuca valdesii Gonz.-Led. et S.D. Koch

** Festuca willdenoviana Schult.

Gouinia isabelensis J.J. Ortiz (NAY)

Gouinia mexicana (Scribn.) Vasey (SLP)

Gouinia papillosa Swallen

Griffithsochloa multifida (Griffiths) G.J. Pierce

** Guadua aculeta Rupr. ex E. Fourn.

** Guadua longifolia (E. Fourn.) R.W. Pohl

Guadua velutina Londoño et L.G. Clark

** Hierochloe mexicana (Rupr. ex E. Fourn.)

Hitchc.

Hilaria annua Reeder et C. Reeder (COL)

* Hilaria belangeri (Steud.) Nash var. belangeri

* Hilaria belangeri (Steud.) Nash var. longifolia (Vasey) Hitchc.

** Hilaria cenchroides Kunth

Hilaria ciliata (Scribn.) Nash

Hilaria hintonii Sohns

Hilaria semplei Sohns (MICH)

* Hilaria swallenii Cory

* Hordeum arizonicum Covas

** Ichnanthus lanceolatus Scribn. et J.G. Sm.

** Isachne pubescens Swallen

Ixophorus palmeri (Vasey) Beetle

** Leersia ligularis Trin. var. breviligulata

(Prodoehl) Pyrah

Leersia ligularis Trin. var. ligularis

Leptochloa aquatica Scribn. et Merr.

* Leptochloa viscida (Scribn.) Beal

Leptocoryphium villaregalis McVaugh et $\mathrm{R}$. Guzmán (JAL)

Luziola gracillima Prodoehl

* Melica montezumae Piper

** Mesosetum blakei Swallen

Mesosetum stoloniferus Swallen

Metcalfia mexicana (Scribn.) Conert

Muhlenbergia aguascalientensis Y. Herrera et de

la Cerda (AGS)
Muhlenbergia alamosae Vasey

Muhlenbergia annua (Vasey) Swallen

* Muhlenbergia appressa C.O. Goodd.

* Muhlenbergia arenacea (Buckley) Hitchc.

Muhlenbergia argentea Vasey (CHIH)

* Muhlenbergia arizonica Scribn.

* Muhlenbergia arsenei Hitchc.

Muhlenbergia articulata Scribn.

Muhlenbergia biloba Hitchc.

Muhlenbergia brandegeei C. Reeder (BCS)

Muhlenbergia brevifolia Scribn. ex Beal

** Muhlenbergia breviligula Hitchc.

* Muhlenbergia brevis C.O. Goodd.

Muhlenbergia breviseta Griseb. ex E. Fourn.

Muhlenbergia brevivaginata Swallen

Muhlenbergia capillipes (M.E. Jones) P.M.

Peterson et Annable (CHIH)

*** Muhlenbergia confusa (E. Fourn.) Swallen

Muhlenbergia crispiseta Hitchc.

Muhlenbergia cualensis Y. Herrera et P.M.

Peterson (JAL)

Muhlenbergia decumbens Swallen (CHIH)

* Muhlenbergia depauperata Scribn.

Muhlenbergia distans Swallen ex Hitchc.

* Muhlenbergia distichophylla (J. Presl) Kunth

* Muhlenbergia dubia E. Fourn.

* Muhlenbergia dumosa Scribn.

Muhlenbergia durangensis Y. Herrera

* Muhlenbergia elongata Scribn. ex Beal

* Muhlenbergia eludens C. Reeder

* Muhlenbergia emersleyi Vasey

Muhlenbergia eriophylla Swallen

Muhlenbergia flavida Vasey

Muhlenbergia flaviseta Scribn. (DGO)

* Muhlenbergia fragilis Swallen

Muhlenbergia gigantea (E. Fourn.) Hitchc.

* Muhlenbergia glauca (Nees) Mez

Muhlenbergia grandis Vasey

Muhlenbergia gypsophila Reeder et C. Reeder

Muhlenbergia hintonii Swallen (MÉX)

Muhlenbergia iridifolia Soderstr. (JAL)

Muhlenbergia jaime-hintonii P.M. Peterson et

Valdés-Reyna (NL)

Muhlenbergia jaliscana Swallen (JAL)

Muhlenbergia laxa Hitchc. (VER)

Muhlenbergia ligulata (E. Fourn.) Scribn. et Merr. 
Apéndice 1. Continuación.

Muhlenbergia longiglumis Vasey

* Muhlenbergia longiligula Hitchc.

Muhlenbergia lucida Swallen

** Muhlenbergia macroura (Kunth) Hitchc.

Muhlenbergia majalcensis P.M. Peterson (CHIH)

Muhlenbergia michisensis Y. Herrera et P.M.

Peterson (DGO)

Muhlenbergia mucronata (Kunth) Trinius

Muhlenbergia mutica (Rupr. ex E. Fourn.) Hitchc.

** Muhlenbergia orophila Swallen

* Muhlenbergia palmeri Vasey

* Muhlenbergia pauciflora Buckley

* Muhlenbergia pectinata C.O. Goodd.

Muhlenbergia pilosa P.M. Peterson, Wippf et

S.D. Jones (MÉX)

** Muhlenbergia plumbea (Trin.) Hitchc.

* Muhlenbergia polycaulis Scribn.

Muhlenbergia pubescens (Kunth) Hitchc.

Muhlenbergia pubigluma Swallen

Muhlenbergia purpusii $\mathrm{Mez}$

** Muhlenbergia quadridentata (Kunth) Trin.

Muhlenbergia reederorum Soderstr.

* Muhlenbergia rigens (Benth.) Hitchc.

** Muhlenbergia robusta (E. Fourn.) Hitchc.

Muhlenbergia schmitzii Hack.

Muhlenbergia scoparia Vasey

Muhlenbergia seatonii Scribn.

* Muhlenbergia setifolia Vasey

* Muhlenbergia sinuosa Swallen

Muhlenbergia speciosa Vasey

Muhlenbergia spiciformis Trin.

* Muhlenbergia straminea Hitchc.

Muhlenbergia stricta (J. Presl) Kunth

Muhlenbergia strictior Scribn. ex Beal

Muhlenbergia subaristata Swallen (DGO)

Muhlenbergia subbiflora Hitchc. (DGO)

Muhlenbergia tarahumara P.M. Peterson et Columbus (CHIH)

* Muhlenbergia texana Buckley

Muhlenbergia trifida Hack.

** Muhlenbergia vaginata Swallen

** Muhlenbergia versicolor Swallen

Muhlenbergia villiflora Hitchc.

* Muhlenbergia virescens (Kunth) Trin.

Muhlenbergia virlettii (E. Fourn.) Soderstr.

Muhlenbergia watsoniana Hitchc.
Muhlenbergia xanthodas Soderstr. (CHIS)

** Olmeca clarkiae (Davidse et R.W. Pohl) Ruiz-

Sanchez, Sosa et Mejía Saules

Olmeca fulgor (Soderstr.) Ruiz-Sanchez, Sosa et

Mejía Saules

Olmeca recta Soderstr. (VER) (P)

Olmeca reflexa Soderstr. (P)

Olmeca zapotecorum Ruiz-Sanchez, Sosa et Mejía Saules (OAX)

Opizia bracteata McVaugh (MICH)

Otatea acuminata (Munro) C.E. Calderón et Soderstr.

Otatea aztecorum (McClure et E.W. Sm.) C.E. Calderón et Soderstr.

Otatea carrilloi Ruiz-Sanchez, Sosa et MejíaSaules (CHIS)

Otatea glauca L. G. Clark et G. Cortés (CHIS)

Otatea reynosoana Ruiz-Sanchez et L.G. Clark

Otatea transvolcanica Ruiz-Sanchez et L.G. Clark

Otatea ximenae Ruiz-Sanchez et L.G. Clark (OAX)

Panicum aztecanum Zuloaga et Morrone

** Panicum bartlettii Swallen

** Panicum biglandulare Scribn. et J. G. Sm.

* Panicum capillarioides Vasey

** Panicum cayoense Swallen

Panicum crateriferum Sohns (GRO)

Panicum decolorans Kunth

** Panicum guatemalense Swallen

* Panicum havardii Vasey

** Panicum incumbens Swallen

Panicum lepidulum Hitchc. et Chase

Panicum longum Hitchc. et Chase (VER)

Panicum macrospermum (Gould) Espejo et López-Ferr.

* Panicum nodatum Hitchc. et Chase

* Panicum pampinosum Hitchc. et Chase

*** Panicum pedicellatum Vasey

* Panicum plenum Hitchc. et Chase

Panicum tamaulipense F.R. Waller et Morden

** Panicum tuerckheimii Hack.

Panicum vaseyanum Scribn. ex Beal

* Pappophorum bicolor E. Fourn.

** Paspalum adoperiens (E. Fourn.) Chase

** Paspalum affine Steud.

Paspalum arsenei Chase 
Apéndice 1. Continuación.

Paspalum chiapense Sánchez-Ken (CHIS)

** Paspalum culiacanum Vasey

** Paspalum cymbiforme E. Fourn.

Paspalum erectum Chase

Paspalum guayanerum Beetle

Paspalum hintonii Chase

Paspalum leptachne Chase (NAY)

Paspalum longicuspe Nash

Paspalum longum Chase ex I.M. Johnst. (COL)

Paspalum luxurians R. Guzmán et L. Rico (JAL)

Paspalum mayanum Chase ex Swallen

Paspalum mutabile Chase

Paspalum nelsonii Chase (CHIS)

Paspalum palmeri Chase

Paspalum sparsum Chase ex Swallen

Paspalum tinctum Chase

Paspalum tolucensis R. Guzmán (MÉX)

** Paspalum variabile (E. Fourn.) Nash

Paspalum virletii E. Fourn.

Paspalum yecorae Sánchez-Ken (SON)

Pennisetum crinitum (Kunth) Spreng.

Pennisetum durum Beal (CHIH)

Pennisetum prolificum Chase

Pentarrhaphis polymorpha (E. Fourn.) Griffiths

** Pentarrhaphis scabra Kunth

** Pereilema ciliatum E. Fourn.

** Peyritschia koelerioides (Peyr.) E. Fourn.

Piptochaetium angustifolium (Hitchc.) Valencia et Costas

Piptochaetium brevicalyx (E. Fourn.) Ricker

Piptochaetium leianthum (Hitchc.) Beetle

* Piptochaetium pringlei (Scribn. ex Beal) Parodi

** Piptochaetium seleri (Pilg.) Henrard

** Piptochaetium virescens (Kunth) Parodi

Poa albescens Hitchc.

Poa bajaensis Soreng (BC)

* Poa bigelovii Vasey et Scribn.

Poa matri-occidentalis P.M. Peterson et Soreng (DGO)

Poa mulleri Swallen (NL)

** Poa orizabensis Hitchc.

Poa ruprechtii Peyr.

Poa seleri Pilg.

Poa sharpii Swallen

* Poa strictiramea Hitchc.

** Poa tacanae Swallen
Pringleochloa stolonifera (E. Fourn.) Scribn.

Reederochloa eludens Soderstr. et H.F. Decker

** Rhipidocladum bartlettii (McClure) McClure

Rhipidocladum martinezii Davidse et R.W. Pohl

\section{(CHIS)}

Schaffnerella gracilis (Benth.) Nash (CHIS)

Schizachyrium gaumeri Nash

Schizachyrium mexicanum (Hitchc.) A. Camus

Schizachyrium muelleri Nash (VER)

Schizachyrium myosurum (J. Presl) A. Camus

* Setaria arizonica Rominger

Setaria latifolia (Scribn.) R.W.A. Herrm.

** Setaria longipila E. Fourn.

Setaria palmeri Henrard (BCS)

* Setaria ramiseta (Scribn.) Pilg.

* Setaria scheelei (Steud.) Hitchc.

* Setaria texana Emery

** Setaria variifolia (Swallen) Davidse

* Setaria villosissima (Scribn. et Merr.) K. Schum.

Setariopsis latiglumis (Vasey) Scribn.

Soderstromia mexicana (Scribn.) C.V. Morton

(GRO)

Sohnsia filifolia (E. Fourn.) Airy Shaw (SLP)

** Sorghastrum brunneum Swallen

Sorghastrum nudipes Nash

Sorghastrum pohlianum Dávila, L.I. Cabrera et Lira (COL)

** Sorghum trichocladum (Rupr. ex Hack.)

Kuntze

* Sphenopholis interrupta (Buckley) Scribn.

Sporobolus atrovirens (Kunth) Kunth

*** Sporobolus buckleyi Vasey

Sporobolus coahuilensis Valdés-Reyna

** Sporobolus erectus Hitchc.

Sporobolus hintonii T.G. Hartley (MÉX)

Sporobolus macrospermus Scribn. ex Beal

* Sporobolus nealleyi Vasey

Sporobolus palmeri Scribn.

Sporobolus potosiensis Wipff et S.D. Jones (SLP)

Sporobolus spiciformis Swallen

Sporobolus splendens Swallen

Sporobolus trichodes Hitchc.

Steinchisma cupreum (Hitchc. et Chase) W.V. Br.

Stipa acuta Swallen (COAH)

Stipa alta Swallen (COAH)

Stipa bracteata Swallen (BC) 
Apéndice 1. Continuación.

Stipa clandestina Hack.

Stipa constricta Hitchc.

* Stipa curvifolia Swallen

Stipa editorum E. Fourn.

* Stipa eminens Cav.

Stipa hirticulmis S.L. Hatch (NL)

Stipa multinodis Scribn. ex Beal

Stipa saxicola Hitchc. (PUE)

Stipa virlettii E. Fourn. (SLP)

Trachypogon palmeri Nash (JAL)

* Trichoneura elegans Swallen

* Tridens texanus (S. Watson) Nash

Triniochloa gracilis Gómez-Sánchez et Gonz.-Led.

Triniochloa laxa Hitchc. (CHIH) (P)

Triniochloa micrantha (Scribn.) Hitchc. (P)

Triniochloa talpensis Gonz.-Led. et Gómez Sánchez (JAL)

** Tripsacum intermedium de Wet et J.R. Harlan

** Tripsacum jalapense de Wet et Brinker

** Tripsacum lanceolatum Rupr. ex E. Fourn.

** Tripsacum maizar E. Hern. et Randolph (A)

Tripsacum manisuroides de Wet et Harlan (CHIS)

** Tripsacum pilosum Scribn. et Merr.

** Tripsacum zopilotense E. Hern. et Randolph (Pr)

** Trisetum angustum Swallen

Trisetum curvisetum Morden et Valdés-Reyna (NL) Trisetum durangense Finot et P.M. Peterson (DGO)

Trisetum filifolium Scribn. ex Beal

Trisetum ligulatum Finot et Zuloaga

Trisetum marthagonzaleziae P.M. Peterson et Finot

Trisetum palmeri Hitchc.

** Trisetum pinetorum Swallen

** Trisetum rosei Scribn. et Merr.

Trisetum spellenbergii Soreng, Finot et P.M.

Peterson

** Trisetum viride (Kunth) Kunth

Trisetum virletii E. Fourn.

Tristachya angustifolia Hitchc. (NAY)

** Tristachya avenacea (J. Presl) Scribn. et Merr.

Tristachya contrerasii R. Guzmán (JAL)

Tristachya laxa Scribn. et Merr.

Tristachya papilosa R. Guzmán (NAY)

Tuctoria fragilis (Swallen) Reeder
Urochloa venosa (Swallen) Morrone et Zuloaga (MICH)

Zea diploperennis H.H. Iltis, Doebley et R.

Guzmán (JAL) (A)

*** Zea luxurians (Durieu et Asch.) R.M. Bird

(OAX)

** Zea mays L. ssp. mexicana H.H. Iltis

Zea perennis (Hitchc.) Reeves et Mangelsd. (JAL) (P)

Zeugites capillaris (Hitchc.) Swallen

Zeugites hackelii Swallen

Zeugites hintonii T.G. Hartley (MÉX)

Zeugites latifolius (Rupr. ex E. Fourn.) Hemsl.

** Zeugites munroanus Hemsl.

Zeugites pittieri Hack. (OAX)

Zeugites sagittatus Hartley (MÉX)

\section{Pontederiaceae}

* Heteranthera mexicana S. Watson (P)

\section{Smilacaceae}

** Smilax aristolochiifolia Mill.

Smilax chiapensis Lundell (CHIS)

Smilax cordifolia Humb. et Bonpl. ex Willd.

Smilax densiflora A. DC. (MÉX)

Smilax erythrocarpa Kunth

** Smilax jalapensis Schltdl.

Smilax moranensis M. Martens et Galeotti

Smilax occidentalis C.V. Morton (GRO)

** Smilax ornata Lem.

Smilax paniculata M. Martens et Galeotti (VER)

Smilax pringlei Greenm.

Smilax purpusii Brandegee (CHIS)

** Smilax regelii Killip et C.V. Morton

** Smilax velutina Killip et C.V. Morton

Triuridaceae

Lacandonia schismatica E. Martínez et Ramos (CHIS) (Pr)

** Triuris brevistylis Donn. Sm.

Xyridaceae

Xyris mexicana S. Watson

Zingiberaceae

** Renealmia pacifica (Maas) Maas et H. Maas 
Espejo: Liliopsida endémicas de México

\title{
APÉNDICE 2
}

Géneros de monocotiledóneas presentes en México. Se indica para cada uno el número total de especies (taxa) presentes en el país, así como el de especies (taxa) endémicas al mismo.

\author{
Agavaceae \\ Agave 128 (169)/113 (139); Beschorneria 8 (9)/6 (7); Furcraea 13/9; Hesperaloe 7 (8)/6; \\ Hesperoyucca 1(3)/1 (2); Manfreda 31/25; Polianthes 17 (20)/16 (19); Prochnyanthes 1/1; \\ Yucca $33(34) / 16$
}

\section{Alismaceae}

Echinodorus 8/1; Hydrocleys 1/0; Limnocharis 2/0; Sagittaria 9 (10)/2

Alliaceae

Allium 27/17; Bessera 2/2; Bloomeria 2/0; Brodiaea 2/0 Dandya 4/4; Dichelostemma 2/0; Diphalangium 1/1; Jaimehintonia 1/1; Milla 10/9; Muilla 1/0; Nothoscordum 3/0; Petronymphe 1/1; Triteleia 1/1; Triteleiopsis 1/1

Alstroemeriaceae

Bomarea $5 / 2$

Amaryllidaceae

Chlidanthus 1/1; Crinum 3/0; Eucharis 1/0; Habranthus 8/7; Hymenocallis 32/31; Sprekelia 2/1; Zephyranthes 34/22

\section{Anthericaceae}

Echeandia $65(66) / 55$

\section{Araceae}

Anthurium 45 (50)/29 (31); Arisaema 2/1; Caladium 1/0; Dieffenbachia 2/0; Dracontium 1/0; Lemna 7/0; Monstera 8/1; Montrichardia 1/0; Philodendron 26 (28)/9; Pistia 1/0; Rhodospatha 1/0; Spathiphyllum 7/2; Spirodela 2/0; Stenospermation 1/0; Syngonium 8/1; Wolffia 2/0; Wolffiella 4/0; Xanthosoma $8 / 2$

\section{Arecaceae}

Acoelorrhaphe 1/0; Acrocomia 1/0; Astrocaryum 1/0; Attalea 3/1; Bactris 2 (3)/0; Brahea 14/12; Calyptrogyne 1/0; Chamaedorea 46/20; Coccothrinax 1/1; Cocos 1/0; Cryosophila 2/1; Desmoncus 3/0; Gaussia 2/1; Geonoma 1/0; Pseudophoenix 1/0; Reinhardtia 2/0; Roystonea 2/0; Sabal 7/4; Synechanthus 1/0; Thrinax 1/0; Washingtonia 2/0

\section{Asphodelaceae}

Aloe 1/0; Asphodelus 1/0; Kniphofia 1/0

\section{Bromeliaceae}

Aechmea 9/2; Androlepis 1/0; Billbergia 2/0; Bromelia 4/2; Catopsis 17/4; Fosterella 1/0; Greigia 2/2; Guzmania 2/0; Hechtia 58/55; Hohenbergia 1/1; Hohenbergiopsis 1/0; Pepinia 2/1; Pitcairnia 47/38; Racinaea 2/1; Tillandsia 232 (239)/172 (176); Ursulaea 2/2; Viridantha 7/7; Vriesea $2 / 1$; Werauhia $8 / 2$

\section{Burmanniaceae}

Apteria 1/0; Burmannia 2/0; Dictyostega 1/0; Gymnosiphon 3/0

Cannaceae

Canna $4 / 0$ 
Apéndice 2. Continuación.

\section{Commelinaceae}

Callisia 14 (16)/7; Commelina 21 (22)/11; Dichorisandra 2/0; Gibasis 14 (18)/11 (15); Gibasoides 1/1; Matudanthus 1/1; Murdannia 1/0; Thyrsanthemum 3/3; Tinantia 9/2; Tradescantia 43 (46)/25; Tripogandra 13/6; Weldenia $1 / 0$

Convallariaceae

Maianthemum 11 (12)/3; Polygonatum 1/0

\section{Costaceae}

Costus $6(7) / 1$

Cyclanthaceae

Asplundia 2/1; Carludovica 2/0; Cyclanthus 1/0; Dicranopygium 1/0

Cymodoceaceae

Halodule 2/0; Syringodium 1/0

\section{Cyperaceae}

Amphiscirpus 1/0; Bolboschoenus 2/0; Bulbostylis 14/4; Calyptrocarya 1/0; Carex 124 (128)/51; Cladium 2/0; Cyperus 85 (88)/16(17); Cypringlea 3/3; Eleocharis 49/12; Fimbristylis 14/3; Fuirena 7 (8)/2; Hypolytrum 1/0; Isolepis 2/0; Karinia 1/1 Kyllinga 3/0; Lipocarpha 5/0; Oxycaryum 1/0; Pycreus 9/1; Remirea 1/0; Rhynchospora 48 (52)/8; Schoenoplectiella 2/0; Schoenoplectus 5/0; Schoenus 1/0; Scirpus 3/0; Scleria 26/2; Uncinia 3/0

\section{Dioscoreaceae}

Dioscorea $75(77) / 50$

Dracaenaceae

Dracaena $1 / 0$

Eriocaulaceae

Eriocaulon 15/9; Paepalanthus 3/2; Syngonanthus 2/1; Tonina 1/0

Funkiaceae

Hesperocallis $1 / 0$

Haemodoraceae

Xiphidium 1/0

Heliconiaceae

Heliconia $14 / 2$

Hyacinthaceae

Chlorogalum 1/0; Hemiphylacus $5 / 5$

Hydrocharitaceae

Egeria 1/0; Halophila 2/0; Hydrilla 1/0; Limnobium 1/0; Najas 3/0; Thalassia 1/0; Vallisneria $1 / 0$

\section{Hypoxidaceae}

Curculigo 1/0; Hypoxis $9 / 6$

\section{Iridaceae}

Ainea 1/1; Alophia 4/2; Calydorea 2/2; Cipura 2/0; Colima 2/2; Cypella 2/2; Eleutherine 1/0; Fosteria 1/1; Iris 1/0; Nemastylis 1 (5)/1 (4); Neomarica 1/0; Orthrosanthus 2/1; Rigidella 4/2; Sessilanthera 3/2; Sisyrinchium 44/28; Tigridia 36 (37)/31 (32); Trimezia 2/1 
Apéndice 2. Continuación.

\author{
Juncaceae \\ Juncus $20(22) / 2 ;$ Luzula $4 / 0$ \\ Juncaginaceae \\ Lilaea $1 / 0$; Triglochin $2 / 0$ \\ Liliaceae \\ Calochortus 22 (23)/14 (15); Fritillaria 1/0; Lilium 3/0 \\ Marantaceae \\ Calathea 13/5; Ischnosiphon 1/0; Maranta 2/0; Stromanthe 2/1; Thalia 1/0 \\ Mayacaceae
}

Mayaca $1 / 0$

Melanthiaceae

Anticlea 6/3; Schoenocaulon 25 (26)/23 (24); Toxicoscordium 2/0; Veratrum 1/0

Musaceae

Musa $1 / 0$

Nolinaceae

Beaucarnea 8/6; Calibanus 2/2; Dasylirion 21/17; Nolina 22/16

Orchidaceae

Acianthera 19/9; Acineta 3/1;Alamania 1 (2)/1 (2); Anathallis 12/4;Arpophyllum 5/2; Artorima 1/1; Aspidogyne 1/0; Aulosepalum 7/4; Barbosella 1/0; Barkeria 15/12; Beloglottis 3/0; Bletia 27/18; Brachystele 3/2; Brassavola 3/0; Brassia 5/1; Bulbophyllum 5/3; Calanthe 1/0; Camaridium 11/4; Campylocentrum 8/0; Catasetum 3/2; Caularthron 1/0; Chondrorhyncha 1/0; Christensonella 1/0; Chysis 4/2; Clowesia 5/4; Cochleanthes 1/0; Coelia 5/0; Comparettia 1/0; Corallorhiza 10/5; Coryanthes 1/0; Corymborkis 1/0; Cranichis 12/3; Cryptarrhena 1/0; Cuitlauzina 7/3; Cyclopogon 10/3; Cycnoches 2/0; Cypripedium 3/1; Cyrtochiloides 1/0; Cyrtopodium 1/0; Deiregyne 14/13; Dendrophylax 1/0; Dichaea 10/0; Dichromanthus 4/1; Dimerandra 1/0; Dinema 1/0; Domingoa 3/2; Dracula 1/0; Dryadella 3/0; Elleanthus 5/1; Encyclia 42 (43)/25 (26); Epidendrum 123/51; Epipactis 1/0; Eriopsis 1/0; Erycina 5/2; Eulophia 1/0; Eurystyles 2/0; Funkiella 9/4; Galeandra 2/1; Galeoglossum 4/4; Galeottia 1/1; Galeottiella 1/1; Gongora 8/1; Goodyera 8/5; Govenia 16/9; Guarianthe 4/0; Habenaria 75/51; Hagsatera 2/2; Helleriella 2/1; Heterotaxis 2/0; Hexalectris 8 (9)/4; Hintonella 1/1; Homalopetalum 3/2; Ionopsis 2/0; Isochilus 11/4; Jacquiniella 7/1; Kefersteinia 1/0; Kegeliella 1/0; Kionophyton 3/2; Kraenzlinella 2/1; Kreodanthus 3/1; Lacaena 1/0; Laelia 11/9; Leochilus 6/2; Lepanthes 63 (64)/45; Lepanthopsis 1/0; Ligeophila 1/0; Liparis 11/6; Lockhartia 3/1; Lycaste 9/1; Lyroglossa 1/0; Macradenia 1/0; Macroclinium 4/2; Malaxis 68/52; Masdevallia 3/0; Maxillaria 3/1; Maxillariella 8/1; Meiracyllium 2/1; Mesadenella 1/0; Mesadenus 4/2; Mexipedium 1/1; Michrochilus 3/1; Microepidendrum 1/1; Mormodes 17 (18)/13 (14); Mormolyca 4/1; Myoxanthus 3/0; Myrmecophila 6 (7)/4; Nemaconia 6/2; Nidema 1/0; Notylia 5 (6)/3; Ocampoa 1/0; Oeceoclades 1/0; Oestlundia 4/4; Oncidium 37 (38)/23; Ornithidium 1/1; Ornithocephalus 7/4; Pachyphyllum 1/1; Palmorchis 1/0; Papperitzia 1/1; Pelexia 6/0; Phloeophila 1/0; Phragmipedium 1/0; Physogyne 3/3; Piperia 1/0; Platanthera 6/3; Platystele 10/0; Platythelys 3/0; Plectrophora 1/0; Pleurothallis 
Apéndice 2. Continuación.

10/0; Pleurothallopsis 1/0; Polystachya 3/1; Ponera 2/1; Ponthieva 12/3; Potosia 3/3; Prescottia 5/2; Prosthechea 46 (47)/24; Pseudogoodyera 1/1; Psilochilus 1/0; Pteroglossa 1/0; Restrepia 2/0; Restrepiella 1/0; Rhetinantha 3/0; Rhyncholaelia 2/0; Rhynchostele 16/9; Rodriguezia 1/1; Rossioglossum 5/3; Sacoila 1/0; Sarcoglottis 9/2; Scaphosepalum 2/0; Scaphyglottis 13/0; Scelochilus 1/0; Schiedeella 12 (14)/9 (10); Sigmatostalix 2/1; Sobralia 9/1; Sotoa 1/1; Specklinia 17/1; Spiranthes 4/1; Stanhopea 14/7; Stelis 59/23; Stellilabium 1/0; Stenorrhynchos 2/0; Svenkoeltzia 4/4; Tamayorkis 4/1; Teuscheria 1/0; Trichocentrum 26/16; Trichopilia 3/1; Trichosalpinx 9/2; Trigonidium 1/0; Triphora 6/2; Tropidia 1/0; Vanilla 9/0; Warczewiczella 1/0; Warrea 1/0; Wullschlaegelia 1/0; Xylobium 3/0; Zeuxine 1/0

\section{Poaceae}

Acroceras 1/0; Aegopogon 3 (4)/1; Agrostis 22/7; Aira 1/0; Allolepis 1/0; Alopecurus 3/0; Andropogon 17/2; Anthephora 1/0; Aristida 37/13; Arthropogon 1/0; Arthrostylidium 2/0; Arundinella 3/1; Aulonemia 1/1; Axonopus 11/4; Blepharidachne 1/0; Blepharoneuron 2/1; Bothriochloa 10/1; Bouteloua 26/8; Brachiaria 10/3; Brachypodium 2/1; Briza 1/0; Bromus 14/4; Buchloe 1/0; Buchlomimus 1/1; Calamagrostis 12/6; Cathestecum 4/2; Cenchrus 9/1; Chaetium 1/0; Chasmanthium 2/1; Chloris 11/0; Chondrosum 11/4; Chusquea 17 (18)/8 (9); Cinna 1/0; Coelorachis 2/0; Coix 1/0; Cottea 1/0; Cryptochloa 1/0; Ctenium 2/2; Cyclostachya 1/1; Cymbopogon 2/0; Cynodon 3/0; Dactylis 1/0; Dactyloctenium 1/0; Danthonia 3/1; Dasyochloa 1/0; Deschampsia 4/1; Dichanthium 2/0; Digitaria 22/4; Dissanthelium 2/0; Distichlis 3/2; Echinochloa 10/3; Echinolaena 2/0; Eleusine 2/0; Elionurus 2/0; Elymus 6/0; Enneapogon 1/0; Enteropogon 2/1; Eragrostis 38/2; Eriochloa 8 (9)/1 (2); Eriochrysis 1/0; Erioneuron 2 (3)/0; Euclasta 1/0; Eustachys 1/0; Festuca 32/17; Glyceria 4/0; Gouinia 5/3; Griffithsochloa 1/1; Guadua 7/1; Gymnopogon 1/0; Gynerium 1/0; Hackelochloa 1/0; Heteropogon 2/0; Hierochloe 1/0; Hilaria 9 (10)/4; Homolepis 1/0; Hordeum 4/0; Hymenachne 1/0; Hyparrhenia 1/0; Ichnanthus 6 (7)/0; Imperata 3/0; Isachne 3/0; Ischaemum 2/0; Ixophorus 2/1; Jouvea 2/0; Koeleria 2/0; Lasiacis 13 (14)/0; Leersia 4 (6)/1; Leptochloa 9 (10)/1; Leptocoryphium 2/1; Leymus 2/0; Limnodea 1/0; Lithachne 1/0; Luziola 5/1; Lycurus 3/0; Melica 5/0; Melinis 1/0; Mesosetum 4/0; Metcalfia 1/1; Microchloa 1/0; Monanthochloe 1/0; Muhlenbergia 117/54; Munroa 1/0; Nassella 1/0; Olmeca 5/5; Olyra 2/0; Opizia 2/1; Oplismenus 3 (4)/0; Orcuttia 1/0; Orthoclada 1/0; Oryza 1/0; Oryzopsis 1/0; Otatea 8/7; Panicum 81 (82)/8; Pappophorum 4/0; Paspalidium 3/0; Paspalum 91/18; Pennisetum 9/3; Pentarrhaphis 2/1; Pereilema 3/0; Peyritschia 3/0; Phalaris 5/0; Pharus 4/0; Phleum 1/0; Phragmites 1/0; Piptochaetium 9/3; Poa 16/6; Polypogon 3/0; Pringleochloa 1/1; Pseudoechinolaena 1/0; Pseudosclerochloa 1/0; Reederochloa 1/1; Reimarochloa 1/0; Rhipidocladum 5/1; Rhynchelytrum 1/0; Rhytachne 1/0; Saccharum 2/0; Sacciolepis 2/0; Schaffnerella 1/1; Schizachyrium 13/4; Scleropogon 1/0; Setaria 24/2; Setariopsis 2/1; Sitanion 1/0; Soderstromia 1/1; Sohnsia 1/1; Sorghastrum 6/2; Sorghum 1/0; Spartina 6/0; Sphenopholis 2/0; Sporobolus 27/9; Steinchisma 1/1; Stenotaphrum 1/0; Stipa 27/10; Streptochaeta 2/0; Streptogyne 1/0; Thrasya 1/0; Trachypogon 1/1; Tragus 1/0; Trichloris 2/0; Trichoneura 1/0; Tridens 5/0; Triniochloa 5/4; Triplasis 1/0; Tripogon 1/0; Tripsacum 12/1; Trisetum 15/8; Tristachya 5/4; Tuctoria 1/1; Uniola 2/0; Urochloa 1/1; Vulpia 2 (3)/0; Zea 4 (5)/2; Zeugites 8/6; Zizaniopsis 1/0; Zuloagaea $1 / 0$ 
Apéndice 2. Continuación.

\section{Pontederiaceae}

Eichhornia 4/0; Heteranthera 9/0; Pontederia 2/0

Potamogetonaceae

Potamogeton 8/0; Ruppia 2/0; Stuckenia 1/0; Zannichelia 1/0

Smilacaceae

Smilax 22/9

Triuridaceae

Lacandonia 1/1; Sciaphila 1/0; Triuris 2/0

Typhaceae

Sparganium 2/0; Typha 2/0

Xyridaceae

Xyris $4 / 1$

Zingiberaceae

Hedychium 2/0; Renealmia 5/0

Zosteracaeae

Phyllospadix 2/0; Zostera 1/0 